\title{
LAPLACE INTEGRALS AND FACTORIAL SERIES IN THE THEORY OF LINEAR DIFFERENTIAL AND LINEAR DIFFERENCE EQUATIONS*
}

\author{
BY
}

W. J. TRJITZINSKY

1. Introduction. Our present object is to carry out application of Laplace integrals (leading to convergent factorial series developments) to the fullest possible extent in the field of linear differential equations,

$$
L(y) \equiv \sum_{k=0}^{n} d_{n-k}(x) y^{(k)}(x)=0 ;
$$

and, secondly, in the field of linear difference equations

$$
L(y) \equiv \sum_{k=0}^{n} d_{n-k}(x) y(x+k)=0 .
$$

In both (A) and (B) the coefficients are given by convergent series of the form

$$
\left.d_{n-k}(x)=\sum_{s=-m}^{\infty} d_{n-k, s} x^{-s / p} \quad \text { (integer } p \geqq 1\right),
$$

and $d_{0}(x), d_{n}(x) \not \equiv 0$. In view of the fact that the two mentioned fields are to a considerable degree analogous, it has been found convenient to give the developments for both of these in a single paper.

Another outstanding method, a method which previously had been applied with complete success in the two indicated fields, as well as in the field of $q$-difference equations, is that based on the study of asymptotic properties of solutions. In this connection the starting point is given by the full sets of formal series solutions (in general divergent), which are known to exist in all cases. Existence of a complete set of formal series solutions for a difference equation (B), as well as other results of a formal character, have been established by G. D. Birkhoff. $†$ For differential equations (A) existence of a full set of formal solutions follows from a work of E. Fabry. $\ddagger$ In the

* Presented to the Society, December 27, 1934; received by the editors April 18, 1934.

$\dagger$ Formal theory of irregular linear difference equations, Acta Mathematica, vol. 54 (1930), pp. 205-246.

† Sur les intégrales des équations différentielles linéaires à coefficients rationnels, Thèse, 1885, Paris. 
asymptotic (analytic) theories of linear differential, difference and $q$-difference equations, respectively, the coefficients in the involved equations are either representable by convergent series of the form (1) or, more generally, they are merely asymptotic (in certain regions) to such, possibly divergent, series. In all essential particulars these three theories have been completely treated (under no restrictions on the formal series solutions) as follows. A joint work by G. D. Birkhoff and W. J. Trjitzinsky gives the developments for difference equations (B).* A paper by Trjitzinsky establishes the general asymptotic theory for $q$-difference equations. $\dagger$ Finally, the general asymptotic theory for differential equations (A) has been developed by Trjitzinsky. $\ddagger$ Broadly speaking, at the basis of the several general asymptotic theories, referred to above, to a very substantial degree lie the ideas and methods due to Birkhoff or inspired by his work.

Important earlier developments, especially in the field of difference equations, are due to C. R. Adams, R. D. Carmichael, H. Galbrun, E. Hilb, O. Perron, S. Pincherle, H. Späth§ and some others.

While the asymptotic theory has been shown to yield full sets of analytic solutions (with appropriate asymptotic properties) in all cases, the situation is different inasmuch as the method of Laplace integrals and application of

* Analytic theory of singular difference equations, Acta Mathematica, vol. 60 (1932), pp. 1-89.

$\dagger$ Analytic theory of linear q-difference equations, Acta Mathematica, vol. 61 (1933), pp. 1-38; also cf. The general case of integro-q-difference equations, Proceedings of the National Academy of Sciences, vol. 18 (1932), pp. 713-719.

$\ddagger$ Analytic theory of linear differential equations, Acta Mathematica, vol. 62 (1934), pp. 167-226.

$\S$ R. D. Carmichael, for instance, chapter IV of the book referred to at the end of the next footnote. C. R. Adams, On the irregular cases of the linear ordinary difference equation, these Transactions, vol. 30 (1928), pp. 507-554. H. Galbrun, Sur certains solutions exceplionnelles d'une équation linéaire aux différences finies, Bulletin de la Société Mathématique de France, vol. 49 (1921), pp. 206-241. E. Hilb, Zur Theorie der linearen Differenzengleichungen, Mathematische Annalen, vol. 85 (1922), pp. 89-98; same title 1, Mathematische Zeitschrift, vol. 14 (1922); same title 2, ibid., vol. 15 (1922), pp. 280-285; same title 3, ibid., vol. 19 (1924), pp. 136-144. O. Perron, Über lineare Differenzengleichungen zweiter Ordnung ..., Heidelberger Sitzungsberichte (mathematisch-physikalische Klasse), No. 17 (1917); Über das Verhalten der Integrale linearer Differenzengleichungen im Unendlichen, Jahresbericht der Deutschen Mathematiker-Vereinigung, vol. 19 (1910), pp. 129-137; Über lineare Differenzengleichungen, Acta Mathematica, vol. 34 (1910), pp. 109-137; Über Systeme . . , Journal für die reine und angewandte Mathematik, vol. 147 (1917), pp. 36-53; Über Summengleichungen und Poincarésche Differenzengleichungen, Mathematische Annalen, vol. 84 (1921), pp. 1-15. S. Pincherle, Sopra una transformazione delle equasioni differenziali lineari . . ., Rendiconti, Istituto Lombardo, (2), vol. 19 (1886), pp. 559-562; Sur la génération de systèmes récurrents . . ., Acta Mathematica, vol. 16 (1892), pp. 341-363; Sulla risoluzione dell' equazione funzionale . .., Memorie, Bologna Accademia, (4), vol. 9 (1888), pp. 181-204; Sulle equazioni alle differenze, Lincei Rendiconti, 1894, pp. 12-17, pp. 99-105; Sulla risoluzione approssimata delle equazioni alle differenze, Lincei Rendiconti, 1898, pp. 230-234. H. Späth, Über das asymptotische Verhalten der Lösungen nichthomogener linearer Differenzengleichungen, Acta Mathematica, vol. 51 (1927), pp. 133-199; same title, Mathematische Zeitschrift, vol. 30 (1929), pp. 487-513. 
factorial series is concerned. This method, whenever successful, enables one to express a formal series solution with the aid of convergent factorial series. In every such case we have a situation when a possibly divergent formal series solution is "summed" by an essentially "exponential" method; however, this method is known to be applicable not in all cases. Basically and predominantly developments of this type rest on numerous important works of N.E. Nörlund* in the theory of factorial series and in connection with application of these series to difference equations. Among the works of others, involving application of Laplace integrals and factorial series to differential and difference equations, outstanding is a sequence of papers due to J. Horn. $\dagger$

As will be seen from the Main Theorems $(\$ \$ 7,12)$ the program of applying Laplace integrals and factorial series to equations (A) and (B) is capable of being extended considerably beyond the results of the earlier writers. On the other hand, certain examples (in $\$ \$ 7$ and 12 ) will demonstrate the fact that these theorems cannot be extended (in a certain sense).

It is also to be noted that, in view of the reciprocal relationship between equations (A) and (B) on one side and corresponding linear systems on the other, results of the type established in these pages will hold for linear systems as well.

Numerous works, which we have not mentioned explicitly, are referred to in the several papers and books indicated in the footnotes of this introduction. However, we have indicated directly the more relevant ones of the previous contributions.

\section{PART I. LINEAR DIFFeRential EQUATIONS}

2. Some preliminary facts concerning differential equations. An equation (A) possesses a full set of formal series solutions $s_{i}(x)$ of the form

$$
s_{i}(x)=e^{Q_{i}(x)} x^{r_{i}} \sigma_{i}(x), \quad Q_{i}(x)=\sum_{r=0}^{l_{i}-1} q_{i}^{i} x^{\left(l_{i}-r\right) / k_{i}} \quad(i=1, \cdots, n),
$$

where the $\sigma_{i}(x)$ are of the form

$$
\sigma_{i}(x)=\sum_{h=0}^{m_{i}} \log ^{h} x{ }_{h \eta^{m_{i}}(x),}
$$

* Some of Nörlund's work is as follows. Acta Mathematica, vol. 37 (1914), pp. 327-387; Leçons si:r les Series d'Interpolation, Paris, 1926 (this book contains an extensive bibliography-pp. 228, 233); Leģons sur les Equations Linéaires aux Différences Finies, Paris, 1929.

$\dagger$ In this connection we shall mention Integration linearer Differentialgleichungen durch Laplacesche Integrale und Fakultätenreihen, Jahresbericht der Deutschen Mathematiker-Vereinigung, vol. 24 (1915), pp. 309-329; Laplacesche Integrale, Binomialkoefficientenreihen und Gammaquotientenreihen in der Theorie der linearen Differentialgleichungen, Mathematische Zeitschrift, vol. 21 (1924), pp. 85-95. 


$$
{ }_{h} \eta^{m_{i}}(x)=\sum_{i=0}^{\infty}{ }_{n} \eta_{i}^{m_{i}} x^{-s / k_{i}} \quad\left(h=0,1, \cdots, m_{i}\right)
$$

here $l_{i}, m_{i}, k_{i}$ are integers $\left(m_{i} \geqq 0 ; k_{i}=r_{i}^{\prime} p\right.$; integer $\left.r_{i}^{\prime} \geqq 1\right)$.

The formal series can be all arranged in logarithmic groups. The exponential factors,

$$
e^{Q(x)} x^{r},
$$

of the series belonging to a particular group are the same.* The elements of such a group can be so ordered,

$$
e^{Q(x)} x^{\tau} \sigma_{i_{1}+1}(x), e^{Q(x)} x^{\tau} \sigma_{i_{1}+2}(x), \cdots, e^{Q(x)} x^{\tau} \sigma_{i_{1}+j}(x),
$$

that $m_{i_{1}+1}=0, m_{i_{1}+2}=1, \cdots, m_{i_{1}+j}=j-1$. A series of the type of (1a) will be termed a $\sigma$-series.

Horn assumes that all the roots of the characteristic equation, corresponding to (A), are simple. Under this supposition all the formal series will be normal; moreover, no $\sigma$-series factor will contain any logarithms (that is, for each formal solution $m_{i}=0$ ). It will be convenient to state his result in a form slightly different from his. By means of a transformation of the type $x^{k}=z$, where $k$ is suitably chosen, the equation (A) is brought to the form of a new equation $\left(A_{1}\right)$, the latter equation differing from the original one in the value of the integer $p$, while $d_{0,0} \neq 0$ and the coefficients of the new equation contain no positive (integral or fractional) powers of $z$. For simplicity the notation originally introduced for the equation (A) will be maintained for the modified equation $\left(A_{1}\right)$. The connection between the results valid for $(A)$ and those valid for $\left(A_{1}\right)$ is obvious. Now, under Horn's hypothesis equation $\left(A_{1}\right)$ possesses a set of $\boldsymbol{n}$ (linearly independent) solutions of the form

$$
e^{Q_{i}(x)} x^{r_{i}} y_{i}(x)
$$$$
(i=1,2, \cdots, n) .
$$

Here the $Q_{i}(x)$ are given by (1) (with $k_{i}=p$ ) and the $y_{i}(x)$ are of the form

$$
y(x)=0 \eta_{0}^{0}+\sum_{w=1}^{p} x^{1-w / p} 0 \eta w^{0}(x),
$$

where the ${ }_{0} \eta_{\omega^{0}}(x)$ are convergent factorial series of the type

$$
{ }_{0 \eta w^{0}}(x)=\sum_{j=0}^{\infty} \frac{0 a_{w: 0}^{0}}{x(x-\gamma) \cdots(x-s \gamma)},
$$

* However, the values of $r$, associated with the same group, may differ by rational fractions. 
$|\gamma|$ is sufficiently great, is the same for all solutions and $\angle \gamma$ is allowed to have any value except certain ones, depending on the roots of the characteristic equation.* Moreover, the series (4a) all converge in a certain half plane. Formally, of course, solutions (3) are compatible with a set of (possibly divergent) formal series solutions.

If the characteristic equation of $\left(A_{1}\right)$ has a simple root, then to this root there will correspond a convergent solution of the form ((3), (4), (4a)). To every simple root of the characteristic equation of $\left(A_{1}\right)$ there corresponds such a convergent solution. This fact, although not demonstrated by Horn, is a rather easy consequence of his work. However, we shall proceed to prove a result reaching much further. Before formulating our objective more precisely the distinction will be first drawn between "normal" and "anormal" formal series solutions. A formal solution $s_{i}(x)$ of $\left(\mathrm{A}_{1}\right)$, as given by (1), (1a), (1b), is said to be normal when $k_{i}=p$ (cf. (1b); the coefficients of $\left(\mathrm{A}_{1}\right)$ are in powers of $\left.x^{1 / p}\right)$. If this is not the case (that is, when the integer $r_{i}^{\prime}$, of $(1 \mathrm{~b})$, is greater than unity) a formal series $s_{i}(x)$ will be said to be anormal. A formal solution of (A) will be normal or anormal according to the nature of the corresponding solutions of $\left(A_{1}\right)$.

In the sequel, unless stated to the contrary, the equation (A) will be taken in the form $\left(A_{1}\right)$. Consider a root $\rho$ of multiplicity $\phi(\geqq 1)$. It may happen that the $\phi$ formal series solutions, corresponding to this root, are all anormal; in some cases some of the $\phi$ formal solutions, associated with such a multiple root, are normal while others are anormal. The third alternative-the one from now on assumed-is that all the formal solutions (1), belonging to the particular root under consideration, are normal. Moreover, it will be assumed that there is just one corresponding logarithmic group: It will be shown, under this assumption, that all the formal solutions under consideration are expressible with the aid of convergent Laplace integrals, leading to convergent factorial series developments. An example in $\$ 7$ will make it evident that in a more general case a result of this type need not hold.

3. Conditions for existence of formal solutions of the type specified in $\$ 2$. The $\phi$ normal formal solutions, corresponding to a root of multiplicity $\phi$, form a logarithmic group (cf. §2) and they may be written as follows:

$$
s_{j}(x)=e^{Q(x)} x^{r} \sum_{h=0}^{j-1} \log ^{h} x_{h \eta^{j-1}(x)}(j=1,2, \cdots, \phi),
$$

where

$$
{ }_{h} \eta^{j-1}(x)=\sum_{s=0}^{\infty}{ }_{h} \eta_{s}^{j-1} x^{-s / p} \quad(h=0,1, \cdots, j-1) .
$$

\footnotetext{
* For the exact situation concerning $\angle \gamma$ cf. Horn's work on differential equations, loc. cit.
} 
The equation $\left(A_{1}\right)$ (cf. beginning of $\$ 2$ ) wil maintain its form*after the transformation

$$
y(x)=e^{Q(x)} x^{r} \bar{y}(x) .
$$

Accordingly, without any loss of generality, it may be assumed that in (1)

$$
e^{Q(x)} x^{r} \equiv 1
$$

Moreover, it will be assumed, as it may be without entailing any loss of generality, that not all the

$$
{ }_{j-1} \eta_{0^{j-1}} \quad(j=1,2, \cdots, \phi)
$$

are zero. However, (1b) implies certain conditions on the coefficients of $\left(A_{1}\right)$. In view of our purpose it will be essential to determine these conditions.

We note first that, when $h(\geqq 0)$ is an integer,

$$
\frac{d^{\nu}}{d x^{\nu}} \log ^{h} x=\sum_{\beta=0}^{h} \log ^{h-\beta} x C_{\beta^{h}}^{h}\left[(-1)^{\nu+\beta} \nu ! g_{\nu, \beta} x^{-\nu}\right],
$$

where, for $\nu \geqq \beta \geqq 1$,

$$
g_{r, \beta}=\sum_{n_{1}, n_{2}, \cdots, n_{\beta}} \frac{1}{n_{1} n_{2} \cdots n_{\beta}},
$$

while $g_{v, \beta}=0$ for $\nu<\beta$. The summation in (2a) is extended over all the positive integral values of $n_{1}, n_{2}, \cdots, n_{\beta}$ such that $n_{1}+\cdots+n_{\beta}=\nu ; \dagger$ moreover, $g_{0,0}=1$ while the $g_{v, 0}$ and the $g_{0, v}$ are all zero for $\nu>0$. In view of (1b), on making use of (2), we get

$$
\begin{aligned}
s_{i}^{(k)}(x) & =\sum_{h=0}^{j-1} \sum_{\nu=0}^{k} C_{\nu}^{k}\left(\frac{d^{\nu}}{d x^{\nu}} \log ^{h} x\right)_{h} \eta^{j-1(k-\nu)}(x) \\
& =\sum_{h=0}^{j-1} \sum_{\beta=0}^{h} \sum_{\beta=0}^{\infty} \sum_{\nu=0}^{k} C_{\nu}^{k} C_{\beta}^{h} C_{k-\nu}^{-s / p}(-1)^{\nu+\beta} \nu !(k-\nu) ! g_{\nu, \beta} \eta_{\eta_{s}}^{j-1} \log ^{h-\beta} x x^{-\alpha / p-k} .
\end{aligned}
$$

Since

$$
\sum_{h=0}^{j-1} \sum_{\beta=0}^{h} a_{h, \beta} l_{h-\beta}=\sum_{h=0}^{j-1} \sum_{\beta=0}^{j-1-h} a_{h+\beta, \beta} l_{h}
$$

it follows that

$$
s_{j}^{(k)}(x)=\sum_{h=0}^{j-1} \sum_{s=0}^{\infty} s_{h, s}^{k, j} \log ^{h} x x^{-s / p-k},
$$

* In particular, the coefficients of the equation will still be in powers of $x^{1 / p}$.

$\dagger$ Formula (2) can be proved, for instance, on the basis of the multinomial theorem of algebra. The $C_{\beta}{ }^{h}$ denote binomial coefficients. 
where

$$
s_{h, \varepsilon}^{k, j}=\sum_{\beta=0}^{j-1-h} \sum_{\nu=0}^{k} C_{\nu}^{k} C_{\beta}^{h+\beta} C_{k-\nu}^{-s / p}(-1)^{p+\beta}{ }_{\nu !}(k-\nu) ! g_{\nu, \beta} \quad h+\beta \eta_{\bullet}^{j-1} .
$$

As a consequence of the easily verifiable relation

$$
\sum_{k=0}^{n} \sum_{\sigma=0}^{\infty} b_{k, \sigma} x^{-\sigma / p-k}=\sum_{\lambda=0}^{\infty} \sum_{w=0}^{p-1} x^{-(\lambda p+w) / p} \sum_{k=0(\leqq n)}^{\lambda} b_{k,(\lambda-k) p+w}
$$

it follows from (4) that formally

$$
\begin{aligned}
L\left(s_{j}(x)\right) & \equiv \sum_{k=0}^{n} \sum_{\lambda=0}^{\infty} d_{n-k, \lambda} x^{-\lambda / p} \sum_{h=0}^{j-1} \sum_{s=0}^{\infty} s_{h, s}^{k, j} \log ^{h} x x^{-(\delta / p+k)} \\
& \equiv \sum_{h=0}^{j-1} \log ^{h} x \sum_{\lambda=0}^{\infty} \sum_{w=0}^{p-1} W_{h: \lambda p+w}^{j} x^{-(\lambda p+w) / p} \quad(j=1,2, \cdots, \phi) .
\end{aligned}
$$

Here

$$
\begin{aligned}
W_{h: \lambda p+w}^{j} & =\sum_{k=0(\leqq n)}^{\lambda} \sum_{s=0}^{(\lambda-k) p+w} s_{h, 8}^{k, j} d_{n-k,(\lambda-k) p+w-s} \\
& =\sum_{k=0(\leqq n)}^{\lambda} \sum_{s=0}^{(\lambda-k) p+w} \sum_{\beta=0}^{j-1-h} \sum_{\nu=0}^{k} C_{\nu}^{k} C_{\beta}^{h+\beta} C_{k-\nu}^{-s / p}(-1)^{p+\beta} \nu !(k-\nu) ! g_{\nu, \beta}
\end{aligned}
$$

$\cdot d_{n-k,(\lambda-k) p+w-\varepsilon h+\beta \eta_{0}^{j-1}}$.

In view of the assumed existence of $\phi$ formal solutions (1) it is inferred that the equations

$$
\begin{gathered}
W_{h: \lambda p+\omega}^{j}=0 \\
{[\lambda=0,1, \cdots ; h=j-1, j-2, \cdots, 0 ; w=0,1, \cdots, p-1 ; j=1,2, \cdots, \phi]}
\end{gathered}
$$

are necessarily formally solvable for the ${ }_{h} \eta_{e^{i-1}}$. Now

$$
\sum_{k=0} \sum_{s=0}^{(\lambda-k) p+w} a_{k, \varepsilon} \eta_{\ell}=\sum_{s=0}^{\lambda p+w} \sum_{k=0}^{k(s)} a_{k, \ell} \eta_{\ell}
$$

where $k(s)=\lambda(s=0,1, \cdots, w), k(s)=\lambda-1 \quad(s=w+1, \cdots, p+w), k(s)$ $=\lambda-2(s=p+w+1, \cdots, 2 p+w), \cdots, k(s)=0(s=(\lambda-1) p+w+1, \cdots$, $\lambda p+w)$. This relation leads to the following:

$$
\begin{aligned}
& \sum_{k=0}^{\lambda} \sum_{0=0}^{(\lambda-k) p+\infty} a_{k, \varepsilon} \eta_{s}=\sum_{\varepsilon=0}^{\infty} \sum_{k=0}^{\lambda} a_{k, \varepsilon} \eta_{s} \\
& +\sum_{m=1}^{\lambda} \sum_{m=\ell^{\prime}}^{m p+w} \sum_{k=0}^{\lambda-m} a_{k, \varepsilon^{\prime} \eta_{\ell}} \quad\left[s^{\prime}=(m-1) p+w+1\right] .
\end{aligned}
$$


In (7) replace $h$ by $j-H(H=1,2, \cdots, j)$. Application of (8) to (7) will then yield

$$
\begin{aligned}
& W_{j-H: \lambda p+w}^{j} \equiv J_{\lambda: w}^{H-1,0}{ }_{j-1} \eta_{0}^{j-1}+\sum_{s=0}^{w} \sum_{\beta=0}^{H-1} C_{\beta}^{j-H+\beta} J_{\lambda: w-\infty}^{\beta,:}{ }_{j-H+\beta}^{j-1} \\
& +\sum_{m=1}^{\lambda} \sum_{s=s^{\prime}}^{m p+w} \sum_{\beta=0}^{H-1} C_{\beta}^{j-H+\beta} J_{\lambda-m: m p+w-s}^{\beta, \bullet}{ }_{j-H+\beta}^{j-1}=0 \\
& {\left[\lambda=0,1, \cdots ; H=1,2, \cdots, j ; w=0,1, \cdots, p-1 ; s^{\prime}\right. \text { as in (8)]. }}
\end{aligned}
$$

Here and in the sequel

$$
\sum_{s=0}^{w} \sum_{\beta=0}^{H-1} b_{s, \beta}=\sum_{s=0}^{w} \sum_{\beta=0}^{H-1} b_{s, \beta}-b_{0, H-1}
$$

moreover,

$$
\begin{aligned}
J_{\sigma: w}^{\beta, Q}=\sum_{k=\beta}^{\sigma} \sum_{\nu=\beta}^{k} C_{\nu}^{k} C_{k \rightarrow \nu}^{-\beta / p}(-1)^{\nu+\beta}{ }_{\nu !(k-\nu) ! g_{\nu, \beta} d_{n-k,(\sigma-k) p+w}} \\
(0 \leqq \beta \leqq \sigma ; s \geqq 0 ; 0 \leqq w \leqq p-1) .
\end{aligned}
$$

It is observed that (9b) defines all the $J_{\sigma: w}^{\beta, s}$ occurring in the second members of (9).

From equations $(9 ; \lambda=0 ; H=1 ; w=0,1, \cdots, p-1)$ we find that

$$
J_{0: w}^{0,0}=0 \quad(w=0,1, \cdots, p-1) ;
$$

this, however, as follows from (9b), is equivalent to

$$
d_{n, w}=0 \quad(w=0,1, \cdots, p-1) .
$$

At this point it will be convenient to introduce the

Definition. $A$ number $d_{i, j}$ will be said to be of index $\sigma$ if $i=n-k$ and $j=(\sigma-k) p+w$, where $0 \leqq k \leqq \sigma$ and $0 \leqq w \leqq p-1$.

Thus the implication of (10) is that all the $d_{i, j}$ of index zero are zero. We note that, as a consequence of the original hypothesis concerning existence of solutions, the characteristic equation of the differential equation $\left(A_{1}\right)$,

$$
E(\rho) \equiv \sum_{k=0}^{n} d_{n-k, 0} \rho^{k}=0,
$$

has a root $\rho=0$ whose multiplicity is precisely $\phi$. Thus

$$
d_{n, 0}=d_{n-1,0}=\cdots=d_{n-\phi+1,0}=0, d_{n-\phi, 0} \neq 0 .
$$


In particular, then, it is to be noted that not all the $d_{i, j}$ of index $\phi$ are zero. Suppose

$$
J_{\sigma: w}^{0,0}=J_{\sigma: w}^{1,0}=\cdots=J_{\sigma: w}^{\sigma, 0}=0 \quad(w=0,1, \cdots, p-1)
$$

for $\sigma=0,1, \cdots, \lambda-1(1 \leqq \lambda \leqq \phi-1)$. From (10) it is seen that (12) is true for $\lambda=1$. We have, by $(9 b)$,

$$
J_{\lambda: v}^{\beta, 0}=\sum_{k=\beta}^{\lambda}(-1)^{k+\beta} k ! g_{k, \beta} d_{n-k,(\lambda-k) p+v} .
$$

Accordingly, by (12), in view of the relations $J_{\sigma: w}^{\sigma, 0}=0$, it follows that

$$
d_{n-\sigma, w}=0 \quad(\sigma=0,1, \cdots, \lambda-1 ; w=0,1, \cdots, p-1) ;
$$

furthermore the relations

$$
J_{\sigma: w}^{\sigma-1,0}=0
$$

yield, by virtue of (14),

$$
d_{n-(\sigma-1), p+\infty}=0 \quad(\sigma=1,2, \cdots, \lambda-1 ; w=0,1, \cdots, p-1) .
$$

From

$$
J_{\sigma: w}^{\sigma-2,0}=0 \quad(\sigma=2, \cdots, \lambda-1 ; w=0, \cdots, p-1),
$$

by (14) and (14a),

$$
d_{n-(\sigma-2), 2 p+w}=0 \quad(\sigma=2, \cdots, \lambda-1 ; w=0, \cdots, p-1) .
$$

On using the relations

$$
J_{\sigma: w}^{\sigma-H, 0}=0 \quad(\sigma=H, H+1, \cdots, \lambda-1 ; w=0,1, \cdots, p-1)
$$

in succession for $H=0,1, \cdots, \lambda-1$ it follows by induction that

$$
\begin{gathered}
d_{n-(\sigma-H), H p+w}=0 \\
(\sigma=H, H+1, \cdots, \lambda-1 ; w=0,1, \cdots, p-1)
\end{gathered}
$$

for $H=0,1, \cdots, \lambda-1$. The subscripts in (14a) can also be considered as extending over the values

$$
H=0, \cdots, \sigma ; \quad \sigma=0,1, \cdots, \lambda-1 ; \quad w=0,1, \cdots, p-1 .
$$

Consequently, on letting in (14a) $H=\sigma-k$, (14a) is seen to be equivalent to

$$
d_{n-k,(\sigma-k) p+w}=0 \quad(k=0,1, \cdots, \sigma ; w=0,1, \cdots, p-1),
$$


where $\sigma=0,1, \cdots, \lambda-1$. That is, (12) implies that all the $d_{i, j}$ of indices $0,1, \cdots, \lambda-1$ are zero. The converse is also true.

All the $d_{i, j}$ in the second members of (9b) are of index $\sigma$; hence $a$ further consequence of (12) would be

$$
\begin{gathered}
J_{\sigma: w}^{\beta, s}=0 \\
(\beta=0,1, \cdots, \sigma ; w=0,1, \cdots, p-1 ; \sigma=0,1, \cdots, \lambda-1 ; s=0,1, \cdots) .
\end{gathered}
$$

In view of (16) from the equations

$$
W_{j-1: \lambda p}^{j}=W_{j-2: \lambda p}^{j}=\cdots=W_{i-\lambda-1: \lambda p}^{j}=0
$$

we obtain in succession

$$
J_{\lambda: 0}^{0,0}=J_{\lambda: 0}^{1,0}=\cdots=J_{\lambda: 0}^{\lambda, 0}=0 .
$$

Suppose now, more generally, that

$$
J_{\lambda: v}^{\beta, 0}=0 \quad(\beta=0,1, \cdots, \lambda)
$$

for $v=0,1, \cdots, w-1(1 \leqq w \leqq p-1)$; in (17a) the relations $(17 \mathrm{~b})$ have been established for $w=1$. Consider the equations

$$
W_{j-1: \lambda p+w}^{j}=W_{j-2: \lambda p+w}^{j}=\cdots=W_{j-(\lambda+1): \lambda p}^{i}=0 .
$$

By (16) the numbers $J_{\lambda-m: m p+w-s}^{\beta, s}$, occurring in the second members of (9), are all zero; moreover, in consequence of (1.7b) the $J_{\lambda: w-s}^{\beta, s}$ (in (9)) are all zero for $w-s \leqq w-1$. Thus, equations (18) are of the form*

$$
W_{j-H: \lambda p+w}^{i} \equiv J_{\lambda: w}^{H-1,0}{ }_{j-1}^{j} \eta_{0}^{j-1}+\sum_{\beta=0}^{H-2} C_{\beta}^{j-H+\beta} J_{\lambda: w}^{\beta, 0}{ }_{j-H+\beta}^{j-1} \eta_{0}^{j-1}=0 .
$$

On using (18a) in succession for $H=1,2, \cdots, \lambda+1$ it is found that

$$
J_{\lambda: w}^{H-1,0}=0 \quad(H=1,2, \cdots, \lambda+1) ;
$$

that is, relations $(17 \mathrm{~b})$ necessarily hold for $v=w$, if they hold for $v=0,1$, $\cdots, w-1(1 \leqq w \leqq p-1)$. Thus, by induction, (12) has been established for $\sigma=0,1, \cdots, \lambda$. This fact completes an induction in a larger sense; that is, (12) is seen to be true for $\lambda=1,2, \cdots, \phi-1$. Just as the two italicized statements in connection with (15) and (16) had been established on the basis of (12) (as originally formulated), we now conclude that all the $d_{i, j}$ of indices $0,1, \cdots, \phi-1$ are zero and that

${ }^{*}$ In (18a) and throughout the paper $\sum_{i=\alpha}^{\beta}=0$ whenever $\beta<\alpha$. 


$$
\begin{gathered}
J_{\sigma: \downarrow}^{\beta, \bullet}=0 \\
(\beta=0,1, \cdots, \sigma ; w=0, \cdots, p-1 ; \sigma=0, \cdots, \phi-1 ; s=0,1, \cdots) .
\end{gathered}
$$

It is noted that, in consequence of (19), (9) holds for $(\lambda=0,1, \cdots$, $\phi-1 ; H=1, \cdots, j ; w=0,1, \cdots, p-1 ; j=1, \cdots, \phi)$, while for these values of the subscripts and superscripts the equalities (9) do not yield any information concerning the coefficients of the formal solutions, whose existence has been postulated. It remains to consider the equations (9) for $\lambda \geqq \phi$. On account of (19), in (9), $m \leqq \lambda-\phi$. For $\lambda=\phi$ these equations give

$$
\begin{aligned}
& J_{\phi: 0}^{0, \omega} j_{j-H \eta_{\omega}^{j-1}}^{j-1}=-\sum_{0=0}^{\omega} \sum_{\beta=0}^{H-1} C_{\beta}^{j-H+\beta} J_{\phi: \omega-0}^{\beta, 0} j-H+\beta \eta_{\phi}^{j-1} \\
& \left(H=1,2, \cdots, j ; w=0,1, \cdots ;(s, \beta) \neq_{.}(w, 0)\right) .
\end{aligned}
$$

In particular,

$$
J_{i}^{0,0}: i_{j-1}^{j-1} \eta_{0}^{-1}=0
$$$$
(j=1, \cdots, \phi)
$$

so that necessarily

$$
J_{\phi: 0}^{0,0}=d_{n, \phi p}=0 .
$$

For $\lambda>\phi,(9)$ yields the relations

$$
\begin{aligned}
& J_{\phi: 0}^{0,(\lambda-\phi) p+\omega}{ }_{j-H \eta_{(\lambda-\phi) p+\omega}^{j-1}}^{j-\infty}=-\sum_{0=0}^{\infty} \sum_{\beta=0}^{B-1} C_{\beta}^{j-B+\beta} J_{\lambda: w-\ell}^{\beta, \ell} ;-H+\beta \eta_{s}^{j-1} \\
& -\sum_{m=1}^{\lambda-\phi} \sum_{i=0^{\prime}}^{m p+\infty} \sum_{\beta=0}^{H-1} C_{\beta}^{j-H+\beta} J_{\lambda-m: m p+\infty-\infty}^{\beta, 0} ;-H+\phi \eta_{\infty}^{j-1} \\
& {[\lambda=\phi+1, \phi+2, \cdots ; H=1,2, \cdots, j ; w=0,1, \cdots, p-1 \text {; }} \\
& j=1,2, \cdots, \phi ;(s, \beta) \neq((\lambda-\phi) p+w, 0) ; s^{\prime} \text { as in (8)]. }
\end{aligned}
$$

The equations (20), (22) are solvable in the following order:

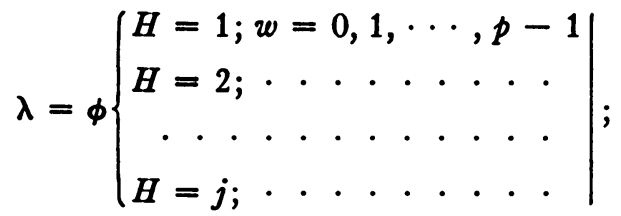

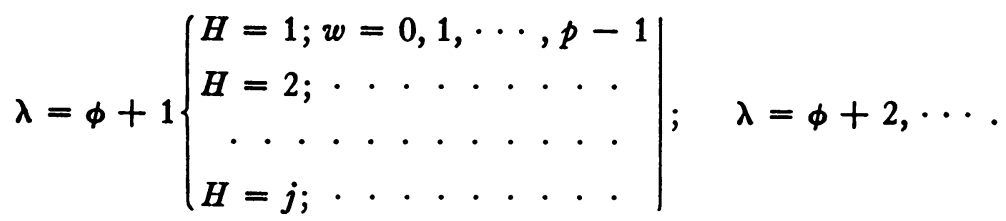


In (20) and (22), by (21) and (9b),

$$
\begin{aligned}
J_{\phi: 0}^{0,(\lambda-\phi) p+w}=\sum_{k=1}^{\phi} C_{k}^{-((\lambda-\phi) p+w) / p} k ! d_{n-k,(\phi-k) p} & \\
& (\lambda=\phi, \phi+1, \cdots ; w=0,1, \cdots, p-1) .
\end{aligned}
$$

Since $d_{n-\phi, 0} \neq 0$ only a finite number of the left members in (24) may vanish. Whenever, for some $w(0 \leqq w \leqq p-1)$ and for some $\lambda(\lambda \geqq 0)$, a number $J_{\phi: 0}^{0,(\lambda-\phi) p+w}=0$ the corresponding ${ }_{j-H} \eta_{(\lambda-\phi) p+w}^{j-1}$ is left undefined. On the other hand, for such a pair of values $(w, \lambda)$, the second member in (22) or, if $\lambda=\phi$, the second member in (20) will be necessarily zero. The ${ }_{j-H+\beta} \eta_{s}{ }^{j-1}$ involved in such a member have known values or some of them may have been previously left undefined. The totality of relationships of such an origin, finite in number, implies certain conditions on the $\eta^{j-1}$ and on the coefficients of $\left(A_{1}\right)$; the latter conditions are necessarily satisfied in view of the assumed existence of solutions of stated type. Some of the $\eta^{i-1}$ may be arbitrary.* The precise nature of the conditions implied by the vanishing of (24) is immaterial for our purposes.

LEMMA 1. Consider a root of multiplicity $\phi$, of the characteristic equation associated with the differential equation $\left(\mathrm{A}_{1}\right)$. In order that, corresponding to this root, there should exist a linearly independent set of $\phi$ formal solutions of type (1), (1a), (1b) the following conditions are necessary and sufficient.

$$
d_{n-\phi, 0} \neq 0, \quad d_{n, \phi p}=0 .
$$

(ii) All the $d_{i, j}$, whose indices (cf. Definition) are $0,1, \cdots, \phi-1$, are zero.

(iii) If any of the $J_{\phi: 0}^{0, \lambda-\phi) p+w}$, defined by (24), are zero (there may be only a finite number of such $J$ ) then the $d_{i, j}$ satisfy conditions implied by the vanishing of the corresponding second members of (22) or (20).

4. The mixed system of differential equations. In the sequel, unless stated to the contrary, it will be assumed that by means of the transformation of the type specified in the beginning of $\$ 3$ the differential equation $\left(A_{1}\right)$ has been brought to such a form that there exist $\phi$ (linearly independent) formal solutions $(1 ; \S 3)$, for which $(1 \mathrm{~b} ; \S 3)$ holds. These solutions may be written as follows:

$$
s_{j}(x)=\sum_{h=0}^{j-1} \log ^{h} x\left({ }_{h} \eta_{0}^{j-1}+\sum_{w=1}^{p} x^{(p-w) / p}{ }_{h \eta_{w}^{j-1}}^{j-1}(x)\right) \quad(j=1, \cdots, \phi),
$$

* In general, whenever some of the numbers (24) are zero, associated with the root, under consideration, of the characteristic equation there will exist more than one formal series solution of $\left(A_{1}\right)$, not involving logarithms. 
where

$$
{ }_{h} \eta_{w}^{j-1}(x)=\sum_{\lambda=0}^{\infty} \underset{h \eta_{\lambda p+w}^{j-1}}{j-\lambda-1} \quad(h=0,1, \cdots, j-1 ; w=1, \cdots, p) .
$$

On the other hand, the coefficients $d_{n-k}(x)$ of $\left(\mathrm{A}_{1}\right)$ may be expressed in the form

$$
\begin{aligned}
d_{n-k}(x) & =d_{n-k, 0}+\sum_{\nu=1}^{p} x^{(p-\nu) / p} d_{n-k, \nu}(x), \\
d_{n-k, \nu}(x) & =\sum_{\lambda=0}^{\infty} d_{n-k, \lambda p+\nu} x^{-\lambda-1} \quad(k=0,1, \cdots, n) .
\end{aligned}
$$

For the purposes at hand it will be essential to establish a "mixed" linear differential system, whose coefficients are in negative integral powers of $x$ and which are formally satisfied by the series (1a). We have

$$
\begin{aligned}
& \frac{d^{k-m} d d x^{k-m}}{}\left({ }_{h} \eta_{0}^{j-1}+\sum_{w=1}^{p} x^{(p-w) / p}{ }_{h \eta_{w}^{j-1}}^{j-x}\right) \\
& \quad={ }_{k} \eta_{0}^{j-1(k-m)}+\sum_{w=1}^{p} \sum_{\delta=0}^{k-m} C_{\delta}^{k-m} C_{\delta}^{(p-w) / p} \delta !_{h}{ }^{j-1(k-m-\delta)}(x) x^{(p-w) / p-\delta} .
\end{aligned}
$$

By (3) and by $(2 ; \S 3)$ it follows from (1) that

$$
\begin{aligned}
s_{j}^{(k)}(x)= & \sum_{h=0}^{j-1} \sum_{m=0}^{k} C_{m}^{k} \sum_{\beta=0}^{h} \log ^{h-\beta} x C_{\beta}^{h}(-1)^{m+\beta} m ! g_{m, \beta} x^{-m} \\
& \times\left[{ }_{h \eta_{0}^{j(k-m)}}+\sum_{w=1}^{p} \sum_{\delta=0}^{k-m} C_{\delta}^{k-m} C_{\delta}^{(p-w) / p} \delta !_{h} \eta_{w}^{j-1(k-m-\delta)}(x) x^{(p-w) / p-\delta}\right] .
\end{aligned}
$$

This may be written in the form

$$
s_{j}^{(k)}(x)=\sum_{h=0}^{j-1} \log ^{h} x\left[S_{1}^{k, j}+S_{2}^{k, j}\right],
$$

where

$$
\begin{aligned}
S_{1}^{k, j} & =\sum_{\beta=0}^{j-h-1} C_{\beta}^{h+\beta}(-1)^{k+\beta} k ! g_{k, \beta} x^{-k} \\
S_{2}^{k, j} & =\sum_{\beta=0}^{j-h-1} \sum_{w=1}^{p} \sum_{m=0}^{k} \sum_{\delta=0}^{m} C_{m-\delta}^{k} C_{\beta}^{h+\beta} C_{\delta}^{k-m+\delta} C_{\delta}^{(p-w) / p}
\end{aligned}
$$

$$
\times(m-\delta) ! \delta !(-1)^{m-\delta+\beta} g_{m-\delta, \beta} \quad{ }_{h+\beta} \eta_{w}^{j-1(k-m)}(x) x^{-m} x^{(p-w) / p} .
$$


Thus

$$
\begin{aligned}
L\left(s_{j}\right) \equiv & \sum_{h=0}^{j-1} \log ^{h} x \sum_{k=0}^{n}\left[S_{1}^{k, j} d_{n-k, 0}+S_{1}^{k, j} \sum_{\eta=1}^{p} x^{(p-\eta) / p} d_{n-k, \nu}(x)\right. \\
& \left.+S_{2}^{k, j} d_{n-k, 0}+S_{2}^{k, j} \sum_{\nu=1}^{p} x^{(p-p) / p} d_{n-k, \nu}(x)\right]=0 .
\end{aligned}
$$

In (5)

(6)

$$
\begin{aligned}
& S_{1}^{k, j} \sum_{v=1}^{p} x^{(p-\nu) / p} d_{n-k, \nu}(x) \\
& \quad=\sum_{w=1}^{p}\left(\sum_{\beta=0}^{i-k-1} C_{\beta}^{h+\beta}(-1)^{k+\beta} k ! g_{k, \beta} d_{n-k, v}(x) x^{-k}\right) x^{(p-w) / p},
\end{aligned}
$$

and, by virtue of the relation

$$
\sum_{w=1}^{p} \sum_{v=1}^{p} a_{w, \nu} x^{(2 p-w-v) / p}=\sum_{w=1}^{p} x^{(p-w) / p}\left[\sum_{v=1}^{w-1} x a_{w-\nu, \nu}+\sum_{\nu=w}^{p} a_{p+w-v, \nu}\right],
$$

we have

$$
\begin{aligned}
& S_{2}^{k, j} \sum_{\nu=1}^{p} x^{(p-v) / p} d_{n-k, \nu}(x) \\
& =\sum_{w=1}^{p} x^{(p-w) / p}\left[\sum_{\nu=1}^{w-1} \sum_{m=0}^{k} \sum_{\beta=0}^{j-h-1} \sum_{\delta=0}^{m} C_{m-\delta}^{k} C_{\beta}^{h+\beta} C_{\delta}^{k-m+\delta} C_{\delta}^{(p-w+\nu) / p}(m-\delta) !\right. \\
& \quad \times \delta !(-1)^{m-\delta+\beta} g_{m-\delta, \beta} d_{n-k, \nu}(x)_{h+\beta} \eta_{w-\nu}^{j-1(k-m)} x^{-(m-1)} \\
& \quad+\sum_{\nu=w}^{p} \sum_{m=0}^{k} \sum_{\beta=0}^{j-h-1} \sum_{\delta=0}^{m} C_{m-\delta}^{k} C_{\beta}^{h+\beta} C_{\delta}^{k-m+\delta} C_{\delta}^{\nu-w}(m-\delta) ! \delta !(-1)^{m-\delta+\beta} \\
& \left.\quad \times g_{m-\delta, \beta} d_{n-k, \nu}(x)_{h+\beta}^{j-1(k-m)}(x) x^{-m}\right] .
\end{aligned}
$$

By (4b), (4c), (6) and (8) we obtain from (5)

(9) $L\left(s_{j}(x)\right) \equiv \sum_{h=0}^{j-1} \sum_{w=1}^{p} \log ^{h} x x^{(p-w) / p} W_{h, w}^{j-1}=0$.

Here

(9a) $\quad W_{h, w}^{j-1}=W_{1}^{h, w, j-1}+W_{2}^{h, w, j-1}+W_{3}^{h, w, j-1}+W_{4}^{h, w, j-1}$

where 


$$
\begin{aligned}
& W_{1}^{h, w, j-1}=\xi(w) \sum_{k=0}^{n} \sum_{\beta=0}^{j-h-1} C_{\beta}^{h+\beta}(-1)^{k+\beta} k ! g_{k, \beta} d_{n-k, 0} x^{-k} \\
&+\sum_{k=0}^{n} \sum_{\beta=0}^{j-h-1} C_{\beta}^{h+\beta}(-1)^{k+\beta} k ! g_{k, \beta} d_{n-k, w}(x) x^{-k} \\
&(\xi(w)=0 \text { for } w \neq p ; \xi(p)=1),
\end{aligned}
$$

(9c)

$$
W_{2}^{h, w, j-1} \equiv \sum_{k=0}^{n} \sum_{m=0}^{n-k} \sum_{\beta=0}^{j-h-1} \sum_{\delta=0}^{m} C_{m-\delta}^{k+m} C_{\beta}^{h+\beta} C_{\delta}^{k+\delta} C_{\delta}^{(p-w) / p}(m-\delta) !
$$

$$
\begin{aligned}
& \times \delta !(-1)^{m-\delta+\beta} g_{m-\delta, \beta} d_{n-k-m, 0}{ }_{h+\beta} \eta_{w}^{j-1(k)}(x) x^{-m} \\
W_{3}^{h, w, j-1} \equiv & \sum_{k=0}^{n} \sum_{m=0}^{n-k} \sum_{\zeta=1}^{w-1} \sum_{\beta=0}^{j-h-1} \sum_{\delta=0}^{m} C_{m-\delta}^{k+m} C_{\beta}^{h+\beta} C_{\delta}^{k+\delta} C_{\delta}^{(p-\zeta) / p} \\
& \times(m-\delta) ! \delta !(-1)^{m-\delta+\beta} g_{m-\delta, \beta} d_{n-k-m, w-\zeta}(x)_{h+\beta} \eta_{\zeta}^{j-1(k)}(x) x^{-(m-1)}
\end{aligned}
$$

and

$$
\text { (9e) } \begin{aligned}
W_{4}^{h, w, j-1} \equiv & \sum_{k=0}^{n} \sum_{m=0}^{n-k} \sum_{\zeta=w}^{p} \sum_{\beta=0}^{j-h-1} \sum_{\delta=0}^{m} C_{m-\delta}^{k+m} C_{\beta}^{h+\beta} C_{\delta}^{k+\delta} C_{\delta}^{(p-\zeta) / p} \\
& \times(m-\delta) ! \delta !(-1)^{m-\delta+\beta} g_{m-\delta, \beta} d_{n-k-m, w+p-5}(x)_{h+\beta \eta}^{j-1(k)}(x) x^{-m} .
\end{aligned}
$$

Now, in view of the formal facts involved, (9) implies that the ${ }_{n} \eta_{v}{ }^{j-1}(x)$ necessarily satisfy the set of equations

$$
\begin{gathered}
W_{h, w}^{j-1}=0 \\
(h=0, \cdots, j-1 ; w=1, \cdots, p ; j=1, \cdots, \phi) .
\end{gathered}
$$

On making use of $(9 a),(9 b),(9 c),(9 d)$ and $(9 e)$ equations $(10)$ are seen to be equivalent to the differential system

$$
T_{h, w}^{j-1} \equiv \sum_{\beta=0}^{j-h-1} \sum_{k=0}^{n} \sum_{\zeta=1}^{p}{ }_{\beta} a_{\zeta, k}^{h, w}(x){ }_{h+\beta} \eta_{\zeta}^{j-1(k)}(x)=g^{h, w, j}(x)
$$

$$
(h=0,1, \cdots, j-1 ; w=1, \cdots, p),
$$

where the ${ }_{n+\beta} \eta \zeta^{j-1}(x)$ are to be regarded as variables; moreover, $\left(\mathrm{A}_{2}\right)$ is formally satisfied by the series (1a). The coefficients in $\left(A_{2}\right)$ are given by

$$
\begin{aligned}
&{ }_{\beta} a_{\zeta, k}^{h, w}(x)=\sum_{m=0}^{n-k} \sum_{\delta=0}^{m} C_{m-\delta}^{k+m} C_{\beta}^{h+\beta} C_{\delta}^{k+\delta} C_{\delta}^{(p-\zeta) / p}(m-\delta) ! \delta ! \\
& \times(-1)^{m-\delta+\beta} g_{m-\delta, \beta} d_{n-k-m, w-\zeta}(x) x^{-(m-1)} \quad(\zeta=1, \cdots, w-1),
\end{aligned}
$$




$$
\begin{aligned}
{ }_{\beta} a_{\zeta, k}^{h, w}(x) & =\sum_{m=0}^{n-k} \sum_{\delta=0}^{m} C_{m-\delta}^{k+m} C_{\beta}^{h+\beta} C_{\delta}^{k+\delta} C_{\delta}^{(p-\zeta) / p}(m-\delta) ! \delta ! \\
\times(-1)^{m-\delta+\beta} g_{m-\delta, \beta} d_{n-k-m, w+p-\zeta}(x) x^{-m} & (\zeta=w+1, \cdots, p),
\end{aligned}
$$$$
{ }_{\beta} a_{w, k}^{h, w}(x)=\sum_{m=0}^{n-k} \sum_{\delta=0}^{m} C_{m-\delta}^{k+m} C_{\beta}^{h+\beta} C_{\delta}^{k+\delta} C_{\delta}^{(p-w) / p}(m-\delta) ! \delta !(-1)^{m-\delta+\beta}
$$

$$
\begin{aligned}
& \times g_{m-\delta, \beta} d_{n-k-m, 0} x^{-m}+\sum_{m=0}^{n-k} \sum_{\delta=0}^{m} C_{m-\delta}^{k+m} C_{\beta}^{h+\beta} C_{\delta}^{k+\delta} C_{\delta}^{(p-w) / p} \\
& \times(m-\delta) ! \delta !(-1)^{m-\delta+\beta} g_{m-\delta, \beta} d_{n-k-m, p}(x) x^{-m}
\end{aligned}
$$

and

$$
g^{h, w, j}(x)=-W_{1}^{h, w, j-1}
$$

Further calculation leads us to conclude that

$$
{ }_{\beta} a_{\zeta, k}^{h, w}(x)=\sum_{\lambda=0}^{\infty}{ }_{\beta} a_{\zeta, k: \lambda}^{h, w} x^{-\lambda}, \quad g^{h, w, j}(x)=\sum_{\lambda=0}^{\infty} g_{\lambda}^{h, w, j} x^{-\lambda},
$$

where the series involved all converge in a neighborhood of infinity, and

$$
\begin{aligned}
{ }_{\beta} a_{\zeta, k: \lambda}^{h, w}= & \sum_{\beta \leqq m \leqq n-k}^{m \leqq \lambda} \sum_{\delta=0}^{m-\beta} C_{m-\delta}^{k+m} C_{\beta}^{h+\beta} C_{\delta}^{k+\delta} C_{\delta}^{(p-\zeta) / p}(m-\delta) ! \delta !(-1)^{m-\delta+\beta} \\
& \times g_{m-\delta, \beta} d_{n-k-m,(\lambda-m) p+w-\zeta} \quad(\zeta=1, \cdots, w-1),
\end{aligned}
$$

$$
\begin{aligned}
{ }_{\beta} a_{\zeta, k: \lambda}^{h, w}= & \sum_{\beta \leqq m \leqq n-k}^{m \leqq \lambda-1} \sum_{\delta=0}^{m-\beta} C_{m-\delta}^{k+m} C_{\beta}^{h+\beta} C_{\delta}^{k+\delta} C_{\delta}^{(p-\zeta) / p}(m-\delta) ! \delta ! \\
& \times(-1)^{m-\delta+\beta} g_{m-\delta, \beta} d_{n-k-m,(\lambda-m) p+w-\zeta} \quad(\zeta=w+1, \cdots, p),
\end{aligned}
$$

$$
{ }_{\beta} a_{w, k: \lambda}^{h, w}=\sum_{\delta=0}^{\lambda-\beta} C_{\lambda-\delta}^{k+\lambda} C_{\beta}^{h+\beta} C_{\delta}^{k+\delta} C_{\delta}^{(p-w) / p}(\lambda-\delta) ! \delta !(-1)^{\lambda-\delta+\beta}{ }_{g_{\lambda-\delta, \beta}} \times d_{n-k-\lambda, 0}
$$

$$
\begin{aligned}
+ & \sum_{\beta \leqq m \leqq n-k}^{m \leqq \lambda-1} \sum_{\delta=0}^{m-\beta} C_{m-\delta}^{k+m} C_{\beta}^{h+\beta} C_{\delta}^{k+\delta} C_{\delta}^{(p-w) / p}(m-\delta) ! \delta ! \\
& \quad \times(-1)^{m-\delta+\beta} g_{m-\delta, \beta} d_{n-k-m,(\lambda-m) p} .
\end{aligned}
$$

It is noted that, according to Definition of $\S 3$, the $d_{i, j}$ occurring in (12a) are of index $\lambda+k$, while those in (12b) are of index $\lambda+k-1$, and the index of the $d_{i, j}$ in (12c) is $\lambda+k$. Thus, by (ii) of Lemma 1 ,

$$
{ }_{\beta} a_{\zeta, k: \lambda}^{h, w}=0 \begin{cases}\lambda+k \leqq \phi-1 & (\zeta \leqq w) \\ \lambda+k \leqq \phi & (\zeta>w) .\end{cases}
$$


It is observed, moreover, that in the summations of (12a) and (12b) we have $\beta \leqq \lambda$ and $\beta \leqq \lambda-1$, respectively; also, in the first summation in (12c) $\lambda-\beta \geqq 0$, while in the second summation $\beta \leqq \lambda-1$. Hence

$$
{ }_{\beta} a_{\zeta, k: \lambda}^{h, w}=0 \begin{cases}\lambda \leqq \beta-1 & (\zeta \leqq w) ; \\ \lambda \leqq \beta & (\zeta>w) .\end{cases}
$$

Finally, since in (12a) and (12b) $\beta \leqq n-k$, we conclude that

$$
{ }_{\beta}^{h, w} a_{\zeta, k: \lambda}^{h, w}=0 \quad(\beta+k>n ; \zeta \neq w) .
$$

In particular,

$$
{ }_{\beta} a_{w, k: \beta}^{h, w}=C_{\beta}^{k+\beta} C_{\beta}^{h+\beta} \beta ! g_{\beta, \beta} d_{n-k-\beta, 0},
$$

so that

$$
{ }_{\beta} a_{w, k: \beta}^{h, w}=0 \quad(k+\beta \leqq \phi-1), \quad{ }_{\beta} a_{w, k: \beta}^{h, w} \neq 0 \quad(k+\beta=\phi) .
$$

Also it is observed that

$$
{ }_{0} a_{\zeta, k: 0}^{h, w}=d_{n-k, w-\zeta} \quad(\zeta=1, \cdots, w-1) .
$$

Lemma 2. Write the $\phi$ formal solutions $(1 ; \S 3),(1 \mathrm{~b} ; \S 3)$, corresponding to a root of multiplicity $\phi$ of the characteristic equation associated with the differential equation $\left(\mathrm{A}_{1}\right)$, in the form (1), (1a). The formal series (1a) (with $j$ fixed) will satisfy a "mixed" differential system $\left(\mathrm{A}_{2}\right)$, whose coefficients are convergent series given by (12), (12a), (12b), (12c), (11c). The coefficients in the series (12) will satisfy (13), (13a), (13b), (14a).

5. The corresponding system of integral equations. In the sequel, unless stated otherwise, we shall write

$$
t=|t| e^{i \vec{t}}
$$

where $i=(-1)^{1 / 2}$; moreover, the integrals

$$
\int_{0}^{\infty}, \quad \int_{0}^{t}
$$

will be supposed to be extended over the ray $(0, \infty)$, of angle $\bar{t}$, and the rectilinear segment $(0, t)$, respectively. The variable $x$ will be so restricted that

$$
\left.\lim _{t}\left|e^{t x} t^{\alpha}\right|=0 \quad \text { (every } \alpha>0\right) \text {, }
$$


when $|t| \rightarrow \infty$ along the ray $(0, \infty)$ of angle $\bar{t}$. As is well known, we have then formally

$$
a(x)=\sum_{s=1}^{\infty} a_{s} x^{-s}=\int_{0}^{\infty} \tilde{a}(t) e^{t x} d t
$$

where

$$
\tilde{a}(t)=\sum_{\nu=0}^{\infty} \tilde{a}_{\nu} t^{\nu-1} ; \quad \tilde{a}_{\nu}=\frac{(-1)^{\nu} a_{\nu}}{(\nu-1) !} .
$$

Thus the series $(1 \mathrm{a} ; \S 4)$ are formally representable as follows:

$$
{ }_{h} \eta_{w}^{j-1}(x)\left(=\sum_{\lambda=0}^{\infty} \underset{h \eta_{\lambda p+w}^{j-1} x^{-\lambda-1}}{i}\right)=\int_{0}^{\infty}{ }_{h} \tilde{\eta}_{w}^{j-1}(t) e^{t x} d t,
$$

where

$$
{ }_{h} \tilde{\eta}_{w}^{j-1}(t)=\sum_{\nu=0}^{\infty}{ }_{h} \tilde{\eta}_{w: \nu}^{j-1} t^{\nu} ; \quad{ }_{h} \tilde{\eta}_{w: \nu}^{j-1}=\frac{(-1)^{\nu+1}}{\nu !}{ }_{h} \eta_{\nu p+w}^{j-1}
$$

With the part within the parenthesis deleted, (3) is to be considered as a transformation which will be applied to the differential system $\left(\mathrm{A}_{2}\right)$. The coefficients in (3a) had been previously defined by $(20,22,23 ; \S 3)$. The latter relations, while useful in proving Lemma 1 , are impracticable for the purpose of establishing convergence of the series (3a) (in the neighborhood of $t=0$ ). Their convergence, however, will be proved in the sequel with the aid of a system of integral equations and a dominant system of integral equations (\$6). Besides, the integral system, to be established below, is to serve a certain other purpose $(\$ 7)$.

In carrying out the transformation (3) it is observed that, formally,

$$
{ }_{h+\beta} \eta_{\zeta}^{j-1(k)}(x)=\int_{0}^{\infty}{ }_{h+\beta} \tilde{\eta}_{\zeta}^{j-1}(t) t^{k} e^{t x} d t
$$

and that, for $\lambda \geqq 1$,

$$
x^{-\lambda}{ }_{h+\beta}^{j-1(k)}(x)=-\int_{0}^{\infty}\left[\int_{0}^{t} \frac{(\tau-t)^{\lambda-1}}{(\lambda-1) !} \tau^{k}{ }_{h+\beta} \tilde{\eta}_{5}^{j-1}(\tau) d \tau\right] e^{t x} d t
$$

provided

$$
\begin{gathered}
{\left[e^{t x} \int_{0}^{t} t^{k}{ }_{h} \tilde{\eta}_{w}^{j-1}(t) d t{ }^{(H)}\right]_{0}^{\infty}=0} \\
(k=0,1, \cdots, n ; h=0, \cdots, j-1 ; w=1, \cdots, p ; H=1,2, \cdots) .
\end{gathered}
$$


In (5) the displayed integration is iterated $H$ times. ${ }^{*}$ Furthermore, by (4a) and $(12 ; \S 4)$

$$
\begin{aligned}
{ }_{\beta}^{h} a_{\zeta, k}^{h, w}(x) & { }_{h+\beta} \eta_{\zeta}^{j-1(k)}(x)=\int_{0}^{\infty} e^{t x}\left[{ }_{\beta} a_{\zeta, k: 0}^{h, w} t^{k}{ }_{h+\beta} \tilde{\eta}_{\zeta}^{j-1}(t)\right. \\
& \left.-\int_{0}^{t}\left(\sum_{\lambda=1}^{\infty}{ }_{\beta} a_{\zeta, k: \lambda}^{h, w} \frac{(\tau-t)^{\lambda-1}}{(\lambda-1) !} \tau^{k}\right)_{h+\beta} \tilde{\eta}_{\zeta}^{j-1}(\tau) d \tau\right] d t .
\end{aligned}
$$

By virtue of (2), we have for the last member of $\left(A_{2}\right)$

$$
\begin{aligned}
& g^{h, w, j}(x)=\int_{0}^{\infty} e^{t x} \tilde{g}^{h, w, j}(t) d t, \\
& \tilde{g}^{h, w, j}(t)=\sum_{\nu=1}^{\infty} \tilde{g}_{\nu}^{h, w, j} t^{\nu-1} ; \tilde{g}_{\nu}^{h, w, j}=\frac{(-1)^{\nu}}{(\nu-1) !} g_{\nu}^{h, w, j} .
\end{aligned}
$$

On account of convergence of the left members in (6a) it is observed that the left members in (6b) are entire functions. On making use of (6) and (6a) it is seen that the system $\left(A_{2}\right)$ is formally satisfied if

$$
\begin{array}{r}
\sum_{\beta=0}^{j-h-1} \sum_{\zeta=1}^{p}{ }_{\beta} b_{\zeta}^{h, w}(t)_{h+\beta} \tilde{\eta}_{\zeta}^{j-1}(t)=\sum_{\beta=0}^{j-h-1} \sum_{\zeta=1}^{p} \int_{0}^{t}{ }_{\beta} c_{\zeta}^{h, w}(t, \tau)_{h+\beta} \tilde{\eta}_{\zeta}^{j-1}(\tau) d \tau+\tilde{g}^{h, w, j}(t) \\
(h \doteq 0, \cdots, j-1 ; w=1, \cdots, p) .
\end{array}
$$

Here

$$
\begin{aligned}
{ }_{\beta} b_{\xi}^{h, w}(t) & =\sum_{k=0}^{n}{ }_{\beta} a_{\zeta, k: 0}^{h, w} t^{k}, \\
{ }_{\beta} C_{\xi}^{h, w}(t, \tau) & =\sum_{\lambda=1}^{\infty} \sum_{k=0}^{n}{ }_{\beta} a_{\zeta, k: \lambda}^{h, w} \frac{(\tau-t)^{\lambda-1}}{(\lambda-1) !} \tau^{k},
\end{aligned}
$$

and the series (7b) are entire in $t$ and $\tau$.

Since by $(13 \mathrm{a} ; \$ 4)$
${ }_{\beta} a_{5, k: 0}^{h, w}=0$
$(\beta>0 ; \zeta \leqq w)$,
${ }_{\beta} a_{\zeta, k: 0}^{h, w}=0$
$(\zeta>w)$,

it follows from (7a) that

$$
\begin{array}{lr}
{ }_{\beta} b_{\zeta}^{h, w}(t)=0 & (\beta>0 ; \zeta \leqq w), \\
{ }_{\beta} b_{\zeta}^{h, w}(t)=0 & (\zeta>w) .
\end{array}
$$

* Those of the steps which at first can be considered as valid only in a formal sense will be finally justified. Thus, absolute convergence of the integrals (3) (for certain suitably defined functions), as well as the relations (5), will be later established. 
Thus, the only functions (7a) which could possibly be not identically zero are the ${ }_{0} b_{\xi^{h}}{ }^{h}(t)(\zeta \leqq w)$; in view of $(7 \mathrm{a})$ and $(15 ; \S 4)$ they are of the form

$$
{ }_{0} b_{\zeta}^{h, w}(t)=\sum_{k=0}^{n} d_{n-k, w-\zeta} t^{k}=b^{w-\zeta}(t) \quad(\zeta=1, \cdots, w) .
$$

In particular, since $d_{n-\phi, 0} \neq 0, b^{0}(t) \not \equiv 0$. Thus the left members in (7) may be replaced by

$$
\sum_{\zeta=1}^{w} b^{w-\zeta}(t){ }_{h} \tilde{\eta}_{\zeta}^{j-1}(t) .
$$

The coefficients in the system (7) will be now investigated in a greater detail. From (7b) we have

$$
\begin{aligned}
{ }_{\beta}^{h, w} C_{\xi}^{h, w}(t, \tau) & =\sum_{\delta=0}^{\infty} \sum_{q=0}^{\infty}{ }_{\beta C_{\xi: s, q}^{h, w} i^{\& q}} \\
& =\sum_{\lambda=1}^{\infty} \sum_{k=0}^{n} \sum_{r=0}^{\lambda-1} C_{r}^{\lambda-1} \frac{(-1)^{\lambda-1-r}}{(\lambda-1) !}{ }_{\beta} a_{\zeta, k: \lambda}^{h, w} t^{\lambda-1-r} \tau^{r+k},
\end{aligned}
$$

so that

$$
{ }_{\beta}^{h C_{\zeta: s, q}^{h, w}}=\sum_{r=0}^{q} C_{r}^{s+r} \frac{(-1)^{s}}{(s+r) !}{ }_{\beta} a_{\zeta, Q-r: s+r+1}^{h, w} \quad(r \geqq q-n) .
$$

Thus, on noting $(13 ; \S 4)$, it is observed that

$$
\begin{aligned}
& \underset{\beta}{{ }_{\beta} C_{\zeta: s, q}^{h, q}}=0 \quad(s+q \leqq \phi-2 ; \zeta \leqq w), \\
& { }_{\beta}^{h C_{\zeta: s, Q}^{h, q}}=0 \quad(s+q \leqq \phi-1 ; \zeta>w) ;
\end{aligned}
$$

moreover, by virtue of $(13 \mathrm{a} ; \S 4)$, the summation in the right member of (11a) is extended so that $r \leqq q$ and

$$
\begin{array}{lr}
r \geqq 0 ; r \geqq q-n ; r \geqq \beta-1-s & \text { (when } s+q \geqq \phi-1 \text { and } \zeta \leqq w), \\
r \geqq 0 ; r \geqq q-n ; r \geqq \beta-s & \text { (when } s+q \geqq \phi \text { and } \zeta>w) .
\end{array}
$$

To establish properties at infinity we first note that, in view of the satisfied conditions of convergence,

$$
\left|{ }_{\beta} a_{5, k: \lambda}^{h, w}\right|, \quad\left|g_{\lambda}^{h, w, j}\right|<R_{\rho}{ }^{\lambda} .
$$

These inequalities are valid for all possible values of the superscripts and subscripts. $\dagger$ By (13), (7b) and (6b) we shall have (exclusive of a small vicinity 
of $\tau=0$ )

$$
\left|{ }_{\beta C_{\xi}}^{h, w}(t, \tau)\right|<R \rho \sum_{\lambda=1}^{\infty} \sum_{k=0}^{n} \frac{(\rho|t-\tau|)^{\lambda-1}}{(\lambda-1) !}|\tau|^{k}<R^{\prime \prime}|\tau|^{n} e^{\rho|t-r|}
$$

and

$$
\left|\tilde{g}^{h, w, j}(t)\right|<R \rho \sum_{\nu=1}^{\infty} \frac{(\rho|t|)^{\nu-1}}{(\nu-1) !}<R^{\prime \prime} e^{\rho|t|} .
$$

Consider the coefficients in the left members of (7) (cf. (10)). It is noted that, since the $d_{n-k, w-\zeta}(\zeta=1, \cdots, w)$ in (9) are of index $k$, application of Lemma 1 will yield the result

$$
\begin{array}{r}
b^{w-\zeta}(t)=t^{\phi} d^{w-\zeta}(t), d^{w-\zeta}(t)=d_{n-\phi, w-\zeta}+d_{n-\phi-1, w-\zeta} t+\cdots \\
+d_{0, w-\zeta} t^{n-\phi} \\
(\zeta=1, \cdots, w) .
\end{array}
$$

On the other hand, since $d_{n-\phi, 0} \neq 0$,

$$
\begin{aligned}
\frac{t^{\phi}}{b^{(0)}(t)}=\frac{1}{d_{n-\phi, 0}+\cdots+d_{0,0} t^{n-\phi}}=d(t)=d_{0}+d_{1} t+\cdots & \\
& \left(d_{0} \neq 0, \neq \infty\right) .
\end{aligned}
$$

Let $P$ denote the complex $t$-plane excluding small sectors, each with vertex at $t=0$ and containing the poles of $d(t)$ (15a) in their interiors.

From (15) and (15a) it follows that

$$
\begin{aligned}
|d(t)| & <d|t|^{-n+\phi}, \\
\left|d^{w-\zeta}(t)\right| & <d|t|^{n-\phi} \quad \quad(\zeta=1, \cdots, w)
\end{aligned}
$$

when $t$ is in $P$, exclusive of a small vicinity of $t=0$.

We shall now proceed to derive a modified integral system. From ( 7$)$, in view of the notation introduced in (15) and (15a),

$$
\begin{aligned}
t_{h}^{\phi} \tilde{\eta}_{w}^{j-1}(t)= & -\sum_{\sigma=1}^{w-1} d(t) b^{w-\zeta}(t)_{h \eta_{\sigma}}^{j-1}(t)+d(t) \bar{g}^{h, w, j}(t) \\
& +\sum_{\beta=0}^{j-h-1} \sum_{\zeta=1}^{p} \int_{0}^{t} d(t)_{\beta C_{\zeta}, w}^{h, w}(t){ }_{h+\beta} \tilde{\eta}_{\zeta}^{j-1}(\tau) d \tau \\
& (h=0, \cdots, j-1 ; w=1, \cdots, p) .
\end{aligned}
$$

It will be demonstrated that (17) can be brought to the form

$$
\begin{array}{r}
t_{h}^{\phi} \tilde{\eta}_{w}^{j-1}(t)=\sum_{\beta=0}^{j-h-1} \sum_{\zeta=1}^{p} \int_{0}^{t}{ }_{\beta}^{* h, w}(t, \tau)_{h+\beta} \tilde{\eta}_{\zeta}^{j-1}(\tau) d \tau+{ }^{*} g^{h, w, j}(t) \\
(h=0, \cdots, j-1 ; w=1, \cdots, p) .
\end{array}
$$


It is noted first that from $(17 ; w=1)$ the equations $\left(\mathrm{A}_{s} ; w=1\right)$ are obtained, with

$$
\begin{gathered}
{ }_{\beta}^{* h, 1}(t, \tau)=d(t){ }_{\beta} C_{\xi}^{h, 1}(t, \tau), \\
{ }^{*} g^{h, 1, j}(t)=d(t) \tilde{g}^{h, 1, j}(t) .
\end{gathered}
$$

Suppose that, for $\sigma=1,2, \cdots, w-1(2 \leqq w \leqq p)$,

$$
t_{h}^{\phi} \tilde{\eta}_{\sigma}^{j-1}(t)=\sum_{\beta=0}^{j-h-1} \sum_{\zeta=1}^{p} \int_{0}^{t}{ }_{\beta}^{* h C_{\zeta}, \sigma}(t, \tau){ }_{h+\beta} \hat{\eta}_{\zeta}^{j-1}(\tau) d \tau+{ }^{*} g^{h, \sigma, j}(t)
$$

Substitution of (19) in (17) will result in equations of form $\left(A_{3}\right)$, where

$$
\begin{gathered}
{ }_{\beta}^{*} C_{\zeta}^{h, w}(t, \tau)=d(t){ }_{\beta} C_{\zeta}^{h, w}(t, \tau)-d(t) d^{\omega-\zeta}(t) \sum_{\sigma=1}^{\omega-1}{ }_{\beta}^{*} C_{\zeta}^{h, \sigma}(t, \tau), \\
{ }^{*} g^{h, \omega, j}(t)=d(t) \tilde{g}^{h, w, i}(t)-\sum_{\sigma=1}^{\omega-1} d(t) d^{\omega-\zeta}(t)^{*} g^{h, \zeta, j}(t) .
\end{gathered}
$$

Relations (20), (20a) have been established for $w=1$ in (18), (18a). Thus, it follows by induction that $(7)$ is equivalent to the system $\left(A_{3}\right)$, where the coefficients are defined in terms of those of (7) by means of the recursion relations (20), (20a).

In view of (15a), of the nature of the coefficients in (7) and of (20) and (20a) it is concluded that the coefficients in $\left(\mathrm{A}_{3}\right)$ are meromorphic functions (in $t$ ), whose $t$-poles are at the non-zero roots of the characteristic equation of $\left(\mathrm{A}_{1}\right)$ [of $(\mathrm{A})$, of course]. The ${ }_{\beta}^{*} c_{\xi}^{h, w}(t, \tau)$ are entire in $\tau$. Accordingly,

$$
\begin{aligned}
& { }_{\beta}^{* h, w}(t, \tau)=\sum_{s=0}^{\infty} \sum_{q=0}^{\infty}{ }_{\beta}^{* h, w} C_{\xi: s, q} i^{q} \tau^{q}, \\
& { }^{*} g^{h, w, j}(t)=\sum_{e=0}^{\infty}{ }^{*} g_{: \ell}^{h, w, j} t ;
\end{aligned}
$$

here the involved series converge for $|t|<\rho^{\prime}$. On using (18), (15a) and (12), (12a) we obtain

$$
\begin{aligned}
& { }_{\beta}^{* h, 1} C_{\zeta:, Q, q}^{h,}=0\left\{\begin{array}{l}
s+q \leqq \phi-2 \\
s+q \leqq \phi-1
\end{array}\right. \\
& (\zeta=1) \text {; } \\
& (\zeta>1) \text {. }
\end{aligned}
$$

Assume the truth of the following (for $\sigma=1$ already established in (22)):

$$
\begin{aligned}
& { }_{\beta}^{*} C_{5: \&, Q}^{h, \mathcal{Q}}=0\left\{\begin{array}{l}
s+q \leqq \phi-2 \\
s+q \leqq \phi-1
\end{array}\right. \\
& (\zeta \leqq \sigma), \\
& (\zeta>1) \text {. }
\end{aligned}
$$


In view of (20), (15), (15a), (12) and (12a), the relations (22a) would imply that

$$
{ }_{\beta}^{* h, w} C_{\zeta: s, q}=0 \begin{cases}s+q \leqq \phi-2 & (\zeta \leqq w), \\ s+q \leqq \phi-1 & (\zeta>w) .\end{cases}
$$

By induction it is inferred at once that (23) holds for all values of the involved subscripts and superscripts.

Let $P_{0}$ denote $P$ (cf. definition following (15a)) with a sufficiently small vicinity of the origin excluded. By (18), (18a), (16), (14) and (14a)

$$
\begin{aligned}
& \left|{ }_{\beta}^{*} C_{\zeta}^{h, 1}(t, \tau)\right|<d R^{\prime \prime}|t|^{\phi}\left|\frac{t}{\tau}\right|^{-n} e^{p|t-\tau|}, \\
& \left|{ }^{*} g^{h, 1, j}(t)\right|<d R^{\prime \prime}|t| \phi|t|^{-n} e^{\rho|t|} \quad(|\tau| \geqq \bar{\rho}>0 ; \bar{\rho} \text { small })
\end{aligned}
$$

for $t$ in $P_{0}$. It will be proved that, for $t$ in $P_{0}$ and $R_{0}$ sufficiently great,

$$
\begin{array}{ll}
\left|{ }_{\beta} C_{\zeta}^{h, w}(t, \tau)\right|<R_{0}|t|^{\phi}\left|\frac{t}{\tau}\right|^{-n} e^{\rho|t-\tau|} & (|\tau| \geqq \bar{\rho}), \\
& \left.{ }^{*} g^{h, w, j}(t)\left|<R_{0}\right| t|\phi| t\right|^{-n} e^{\rho|t|}
\end{array}
$$

for all values of the involved subscripts and superscripts. Suppose that, for some positive $R_{1}$, for $t$ in $P_{0}$ and $|\tau| \geqq \bar{\rho}$,

$$
\begin{aligned}
& \left|{ }_{\beta}^{* h, \sigma}(t, \tau)\right|<R_{1}|t|^{\phi}\left|\frac{t}{\tau}\right|^{-n} e^{\rho|t-\tau|}, \\
& \left|{ }^{*} g^{h, \sigma, j(t)}\right|<R_{1}|t| \phi|t|^{-n} e^{\rho|t|} \\
& (\sigma=1, \cdots, w-1 ; 2 \leqq w \leqq p) .
\end{aligned}
$$

By (20), (16), (14), (16a) we would have in virtue of (26)

$$
\left|{ }_{\beta}^{*} C_{\zeta}^{h, w}(t, \tau)\right|<|t|^{\phi}\left|\frac{t}{\tau}\right|^{-n} e^{\rho|t-\tau|} d\left(R^{\prime \prime}+d p R_{1}\right) .
$$

On the other hand, in view of (20a), (16), (14a) and (16a) the implication of (26a) would be

$$
\left|{ }^{*} g_{, w, i}(t)\right|<|t| \oplus|t|^{-n} e^{\infty} \mid t 1 .
$$

Here (27) and (27a) have been established for $t$ in $P_{0}$ and for $|\tau| \geqq \bar{p}$. The truth of (25) and (25a) follows by induction.

We formulate the above developments of this section in the Lemma. 
LEMma 3. The formal series ${ }_{n} \bar{\eta}_{w^{j-1}}(t)$ ( $c f$. (3a)), connected with the formal series $(1 ; \S 4)$ by means of $(1 \mathrm{a} ; \S 4)$, satisfy a certain integral system $\left(\mathrm{A}_{3}\right)$. The coefficients of this system are defined by convergent series (21), (21a); they are meromorphic functions in $t$, whose only finite $t$-singularities are poles at the nonzero roots of the characteristic equation of $\left(\mathrm{A}_{1}\right)$ (of $(\mathrm{A})$, also). The ${ }_{\beta}^{*} C_{\zeta}^{h, w}(t, \tau)$ are entire in $\tau$. Moreover, inasmuch as properties at $(t=0, \tau=0)$ are concerned, these coefficients satisfy (23). On the other hand, essential properties at infinity are characterized by (25) and (25a); these inequalities are valid for $|\tau| \geqq \bar{\rho}>0$ ( $\bar{\rho}$ sufficiently small) and for $t$ in $P_{0}$ (cf. definition preceding (24)).

6. The dominant system of integral equations. In proving convergence of the formal solutions ${ }_{h} \tilde{\eta}_{w}{ }^{j-1}(t)(3 \mathrm{a} ; \S 5)$, of the integral system $\left(\mathrm{A}_{8}\right)$, the method of successive approximations (used with success by $\mathrm{J}$. Horn in analogous, but simpler, situations) leads to apparently unsurmountable algebraic difficulties. Accordingly, a different method will be employed. We shall establish a dominant system of integral equations; that is, a system from the convergence of whose solutions convergence of a set of solutions of $\left(\mathrm{A}_{8}\right)$ can be inferred. It will be necessary, first, to derive in detail the relations satisfied by the coefficients of the ${ }_{h} \tilde{\eta}_{w}{ }^{j-1}(t)$. On using $(3 \mathrm{a} ; \S 5)$ and $(21 ; \S 5)$ it follows that

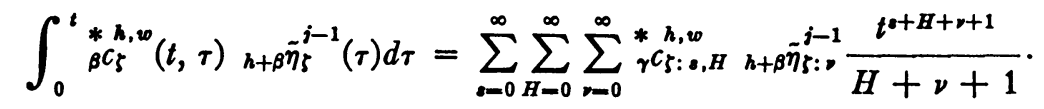

Also, by $(3 a ; \S 5)$,

$$
t_{h}^{\phi} \tilde{\eta}_{w}^{j-1}(t)=\sum_{q=\phi-1}^{\infty}{ }_{h \tilde{\eta}_{w: q-\phi+1}^{j-1}}^{i+1}
$$

Substitution of (1), (1a) and $(21 \mathrm{a} ; \S 5)$ in $\left(\mathrm{A}_{3}\right)$ gives, after a suitable arrangement of terms,

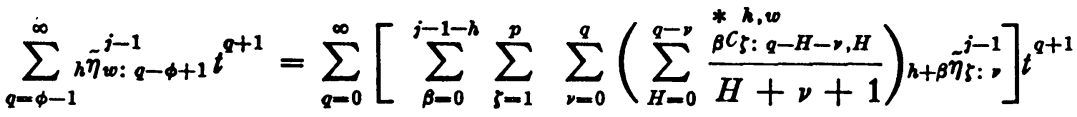

$$
\begin{aligned}
& +\sum_{q}^{\infty} g_{q+1}^{h, w, i} t^{q+1} \\
& =\sum_{q}{ }_{h} f_{w: q} t^{q+1}(h=0,1, \cdots, j-1 ; w=1, \cdots, p) .
\end{aligned}
$$

By virtue of $(23 ; \S 5)$ it is concluded that in (2)

$$
{ }_{h} f_{w: q}=0 \quad(h=0,1, \cdots, j-1 ; w=1, \cdots, p ; q \leqq \phi-2) .
$$

Thus (2) is formally possible if 


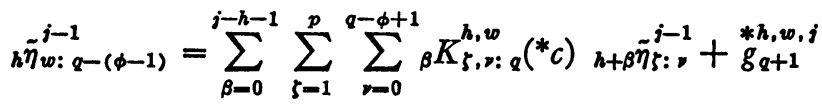

$$
\begin{aligned}
& (q=\phi-1, \phi, \phi+1, \cdots ; h=0,1, \cdots, j-1 ; w=1, \cdots, p),
\end{aligned}
$$

where, for $\zeta>w$, the summation with respect to $\nu$ is extended from $\nu=0$ to $\nu=q-\phi$, and

$$
{ }_{\beta} K_{\zeta, \nu: Q}^{h, w}\left({ }^{*} c\right)=\sum_{H=0}^{q-\gamma} \frac{1}{H+\nu+1}{ }_{\beta}^{*} C_{\zeta: Q-\nu-H, H}^{h, w} .
$$

From (3) and (3a) it is inferred that

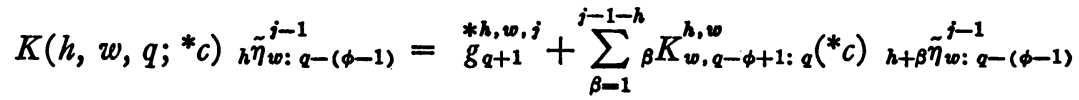

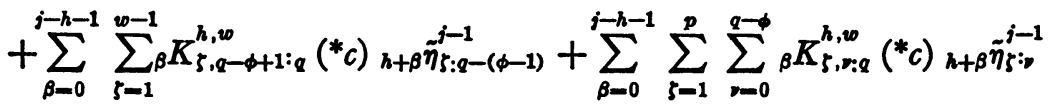

$$
\begin{aligned}
& (h=0,1, \cdots, j-1 ; w=1, \cdots, p ; q=\phi-1, \phi, \phi+1, \cdots),
\end{aligned}
$$

where

$$
K\left(h, w, q ;{ }^{*} c\right)=1-\sum_{H=0}^{\phi-1} \frac{1}{H+q-\phi+2}{ }_{0}^{* h, w} C_{w: \phi-1-H, H}^{h,}
$$

We note that the $K\left(h, w, q ;{ }^{*} c\right)$ may vanish, if at all, only for a finite number of values of $h, w, q$. The equation (4) may be solved in succession according to the scheme

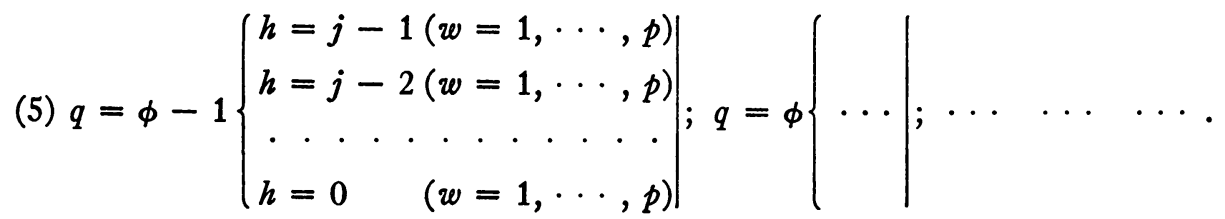

If some of the $K\left(h, w, q ;{ }^{*} c\right)$ are zero, a remark entirely analogous to that following $(24 ; \S 3)$ will be valid, and some of the $\tilde{\eta}^{j-1}$ may be arbitrary (this, of course, corresponds to the possible arbitrariness of some of the $\left.\eta^{j-1}\right)$. It is also observed that none of the ${ }^{*} c$, occurring in (4a), are involved in the second members of (4).

Let $(\overline{4})$ denote the set of equations (4), where the $\tilde{\eta}^{j-1}$, the ${ }^{*} g_{q+1}^{h, w}$ and the ${ }^{*} c$ are replaced by $\bar{\eta}^{j-1}, \bar{g}_{q+1}^{h, w, j}$ and $\bar{c}$, respectively. Choose

$$
\begin{gathered}
{ }_{0 \bar{c}_{w: \phi-1-H, H}^{h, w}=c>0} \\
(H=0,1, \cdots, \phi-1 ; w=1, \cdots, p ; h=0, \cdots, j-1),
\end{gathered}
$$


where $c$ is sufficiently small so that

(6a) $K(h, w, q ; \bar{c})>0 \quad(q \geqq \phi-1 ; w=1, \cdots, p ; h=0, \cdots, j-1)$.

By (6a) and since

$$
\lim _{q \rightarrow \infty} K\left(h, w, q ;{ }^{*} c\right)=\lim _{q \rightarrow \infty} K(h, w, q ; \bar{c})=1,
$$

we have, excluding the values $h, w, q$ for which $K\left(h, w, q ;{ }^{*} c\right)=0$,

$$
\begin{aligned}
0< & \frac{K(h, w, q ; \bar{c})}{\left|K\left(h, w, q ;{ }^{*} c\right)\right|}<B(>1) \\
& \quad(w=1, \cdots, p ; h=0, \cdots, j-1 ; q \geqq \phi-1) .
\end{aligned}
$$

Furthermore, take

$$
\begin{aligned}
{ }_{\beta \bar{C}_{\zeta: \rho, u}^{h, w}} & =0 \begin{cases}\rho+u \leqq \phi-2 & (1 \leqq \zeta \leqq w), \\
\rho+u \leqq \phi-1 & (w<\zeta \leqq p) ;\end{cases} \\
\bar{g}_{q+1}^{h, w} & =0
\end{aligned}
$$

on the other hand, let the other $\bar{c}$ and $\bar{g}$ (that is, those not referred to in (6), (7), (7a)) be positive and greater than the absolute values of the corresponding ${ }^{*} c$ and ${ }^{*} g$. Since $K(h, w, q ; \bar{c}) \neq 0$ for any of the involved values of $h, w, q$ it is observed that equations (4) determine all the ${ }_{h} \bar{\eta}_{w: l}^{j-1}$ uniquely. By virtue of (6a), and in view of the assumptions concerning the $\bar{c}$ and the $\bar{g}$ we conclude that the ${ }_{k} \bar{\eta}_{w: l}^{j-1}$ are all positive. We now prove that, with $B$ of (6b) sufficiently great,

$$
\begin{aligned}
& \left|{ }_{h} \tilde{\eta}_{w: l}^{j-1}\right|<C_{h{ }_{h} \bar{\eta}_{w: l}^{j-1}}^{l+1} \\
& \left(C=B^{i p} ; h=0,1, \cdots, j-1 ; w=1, \cdots, p ; l=0,1, \cdots\right) .
\end{aligned}
$$

Of course, when there is any arbitrariness in the determination of the $\tilde{\eta}^{i-1}$, we shall take some particular set of the $\tilde{\eta}^{j-1}$. By (4a) there exists an integer $q_{0} \geqq \phi-1$ such that

$$
\begin{gathered}
K\left(h, w, q ;{ }^{*} c\right) \neq 0 \\
\left(h=0,1, \cdots, j-1 ; w=1, \cdots, p ; q=q_{0}, q_{0}+1, \cdots\right) .
\end{gathered}
$$

Let the $\tilde{\eta}^{j-1}$ be assigned some particular admissible set of values. There will exist then a number $B$ so that not only (6b) holds but also

$$
\begin{gathered}
\left|{ }_{h \tilde{\eta}_{w: l}}^{j-1}\right|<C^{l+1}{ }_{h}^{i_{w: l}^{j-1}} \\
\left(C=B^{i p} ; h=0,1, \cdots, j-1 ; w=1, \cdots, p ; l=0,1, \cdots, q_{0}-\phi+1\right) .
\end{gathered}
$$

The essential fact is observed that, for $q \geqq q_{0}$, equations (4) define the 
${ }_{n} \tilde{\eta}_{w: q-\phi+1}^{j-1}\left(q \geqq q_{0}\right)$ uniquely in terms of certain other $\tilde{\eta}^{j-1}$, previously defined or selected in a definite manner. Suppose now that

$$
\begin{gathered}
\left|{ }_{h} \tilde{\eta}_{w: l}^{j-1}\right|<C^{l+1}{ }_{h}{ }_{\eta_{w: l}^{j-1}}^{j-1} \\
(h=0,1, \cdots, j-1 ; w=1, \cdots, p ; l=0, \cdots, q-\phi)
\end{gathered}
$$

where $q-1 \geqq q_{0}$ (this secures $q-\phi \geqq q_{0}-\phi+1$ ).

Since the ${ }_{\beta} K_{\zeta, i: \sigma}\left({ }^{*} c\right)$ are forms, with positive coefficients, linear in the ${ }^{*} c$, it follows that

$$
\left|{ }_{\beta} K_{\zeta, i: \sigma}^{h, w}\left({ }^{*} c\right)\right|<{ }_{\beta} K_{\zeta, i: \sigma}^{h, w}(\bar{c})
$$

for all involved values of the subscripts and superscripts. In view of (10) and (6b), application of $(9 \mathrm{~b})$ to (4) would yield the inequalities

$$
\begin{aligned}
\left|{ }_{h} \tilde{\eta}_{w: q-\phi+1}^{j-1}\right|< & \frac{B}{K(h, w, q ; \bar{c})}\left[\bar{g}_{q+1}^{h, w, j}+\left.\sum_{\beta=1}^{j-1-h}{ }_{\beta} K_{w, q-\phi+1: q}^{h, w}(\bar{c})\right|_{h+\beta} \tilde{\eta}_{w: q-\phi+1}^{j-1} \mid\right. \\
& +\left.\sum_{\beta=0}^{j-h-1} \sum_{\zeta=1}^{w-1}{ }_{\beta} K_{\zeta, q-\phi+1: q}^{h, w}(\bar{c})\right|_{h+\beta} \tilde{\eta}_{\zeta: q-\phi+1}^{j-1} \mid \\
& \left.+\sum_{\beta=0}^{j-h-1} \sum_{\zeta=1}^{p} \sum_{\nu=0}^{q-\phi}{ }_{\beta} K_{\zeta, v: q}^{h, w}(\bar{c})_{h+\beta} \bar{\eta}_{\zeta:,}^{j-1} C^{p+1}\right]
\end{aligned}
$$

From $(11 ; h=j-1 ; w=1)$

$$
\begin{aligned}
& \left|\underset{j-1}{j-1} \tilde{\eta}_{1: q-\phi+1}^{j-1}\right|<\frac{B}{K(j-1,1, q ; \bar{c})}\left[\bar{g}_{q+1}^{j-1,1, j}+\sum_{\zeta=1}^{p} \sum_{\nu=0}^{q-\phi}{ }_{0} K_{\zeta, \nu: q}^{j-1,1}(\bar{c})_{j-1} \bar{\eta}_{\zeta: \nu}^{j-1} C^{p+1}\right] \\
& <B C^{q-\phi+1}{ }_{j-1}^{i \eta_{1: q-\phi+1}^{j-1}} \text {. }
\end{aligned}
$$

The latter inequality is obtained using the fact that $C>1$ and that the expression within the brackets in the second member of (11a) would be equal to $K(j-1,1, q ; \bar{c})$ if $C$ were replaced by unity. This type of reasoning is employed in the sequel, but no explicit reference will be made to it. Assume that

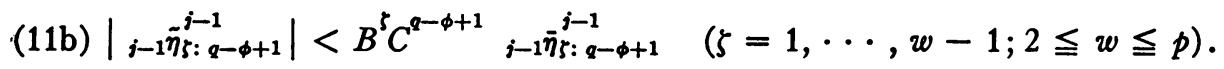
By virtue of (11b), (11) would imply that

$$
\begin{aligned}
\left|{ }_{j-1} \tilde{\eta}_{w: q-\phi+1}^{j-1}\right|< & \frac{B^{w} C^{q-\phi+1}}{K(j-1, w, q ; \bar{c})}\left[\bar{g}_{q+1}^{j-1, w} B^{-(w-1)} C^{-(q-\phi+1)}\right. \\
& +\sum_{\zeta=1}^{w-1}{ }_{0} K_{\zeta, q-\phi+1: q}^{j-1, w}(\bar{c})_{j-1}^{j-1} \bar{\eta}_{\zeta: q-\phi+1}^{j-1} B^{-(w-1-\zeta)} \\
& \left.+\sum_{\zeta=1}^{p} \sum_{v=0}^{q-\phi}{ }_{0} K_{\zeta, v: q}^{j-1, w}(\bar{c})_{j-1}^{j-1} \bar{\eta}_{\zeta: \nu}^{j-1} C^{-(q-\phi-\nu)} B^{-(w-1)}\right] \\
< & B^{w} C^{q-\phi+1}{ }_{j-1}^{j-1} \bar{\eta}_{w: q-\phi+1}^{j-1} .
\end{aligned}
$$


By induction it is seen that $(11 \mathrm{~b})$ is valid for $\zeta=1, \cdots, p$. In particular

$$
\left|{ }_{j-1} \tilde{\eta}_{\zeta: q-\phi+1}^{j-1}\right|<B^{p} C^{q-\phi+1}{ }_{j-1}^{i \bar{\eta}_{\zeta: q-\phi+1}^{j-1}} \quad(\zeta=1, \cdots, p) .
$$

Suppose that, for $\lambda=j-1, j-2, \cdots, h+1(0 \leqq h \leqq j-2)$,

$$
\left|\lambda \bar{\eta}_{\zeta: q-\phi+1}^{j-1}\right|<B^{(j-\lambda) p} C^{q-\phi+1}{ }_{\lambda \bar{\eta}_{\zeta: q-\phi+1}^{j-1}}^{j} \quad(\zeta=1, \cdots, p) .
$$

By (11) we then would have

$$
\begin{aligned}
& \left|\underset{h \tilde{\eta}_{1: q-\phi+1}^{j-1}}{\mid-1}\right|<\frac{B}{K(h, 1, q ; \bar{c})}\left[\bar{g}_{q+1}^{h, 1, j}+\sum_{\beta=1}^{j-1-h}{ }_{\beta} K_{1, q-\phi+1: q}^{h, 1}(\bar{c})_{h+\beta} \bar{\eta}_{1: q-\phi+1}^{j-1}\right. \\
& \left.\times C^{q-\phi+1} B^{(j-h-\beta) p}+\sum_{\beta=0}^{j-h-1} \sum_{\zeta=1}^{p} \sum_{\eta=0}^{q-\phi}{ }_{\beta} K_{\zeta,: q Q}^{h, 1}(\bar{c})_{h+\beta} \bar{\eta}_{\zeta: \nu}^{j-1} C^{p+1}\right] \\
& <B B^{(j-h-1) p} C^{q-\phi+1}{ }_{h \eta_{1: q-\phi+1}^{j-1}}^{i-1}
\end{aligned}
$$

Assume now that

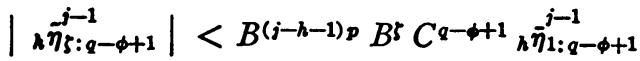

$$
\begin{aligned}
& (\zeta=1, \cdots, w-1 ; 2 \leqq w \leqq p) ;
\end{aligned}
$$

in (14a) these inequalities have been demonstrated for $w=2$. From (11) we would have

(15a)

$$
\begin{aligned}
& \left|\underset{h \eta_{w: q-\phi+1}^{j-1}}{j-1}\right|<\frac{B}{K(h, w, q ; \bar{c})}\left[\bar{g}_{q+1}^{h, w, j}+\sum_{\beta=1}^{j-1-h} K_{w, q-\phi+1: q}^{h, w}(\bar{c})\right. \\
& \times_{h+\beta} \bar{\eta}_{w: q-\phi+1}^{j-1} B^{(j-h-\beta) p} C^{q-\phi+1}+\sum_{\beta=1}^{j-h-1} \sum_{\zeta=1}^{w-1}{ }_{\beta} K_{\zeta, q-\phi+1: q}^{h, w}(\bar{c})_{h+\beta} \bar{\eta}_{\zeta: q-\phi+1}^{j-1} \\
& \times B^{(j-h-\beta) p} C^{q-\phi+1}+\sum_{\zeta=1}^{w-1}{ }_{0} K_{\zeta, q-\phi+1: q}^{h, w}(\bar{c})_{h} \bar{\eta}_{\zeta: q-\phi+1}^{j-1} B^{(j-h-1) p} \\
& \left.\times B^{\zeta} C^{q-\phi+1}+\sum_{\beta=0}^{j-h-1} \sum_{\zeta=1}^{p} \sum_{\nu=0}^{q-\phi}{ }_{\beta} K_{\zeta, \nu: q}^{h, \infty}(\bar{c})_{h+\beta \bar{\eta}_{\zeta: \nu}^{j-1}} C^{p+1}\right] \\
& <B^{(j-h-1) p} B^{w} C^{q-\phi+1}{ }_{h \eta_{w: q-\phi+1}}^{i-1} .
\end{aligned}
$$

Thus (15) holds for $\zeta=w$ and, consequently, for $\zeta=1, \cdots, p$. Whence it follows that

$$
\left|{ }_{h} \tilde{\eta}_{\xi: q-\phi+1}^{j-1}\right|<B^{(j-h) p} C^{q-\phi+1}{ }_{h \bar{\eta}_{\zeta: q-\phi+1}^{j-1}}^{j-1} \quad(\zeta=1, \cdots, p) .
$$

This, however, means that (14) would hold for $\lambda=h$ if (14) holds for $\lambda=j-1$, $j-2, \cdots, h+1$. For $\lambda=j-1$ (14) is observed to be true; thus, (14) holds for 
$\lambda=j-1, j-2, \cdots, 0$. The latter fact implies that

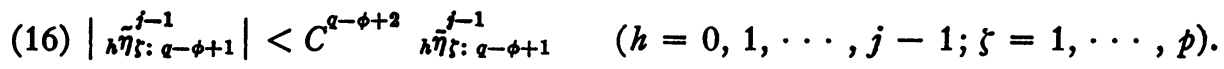

Since (16) is a consequence of (9b), inequalities (8) are seen to be proved. We observe that the following fact has been demonstrated.

Let the $\bar{c}$ and the $\bar{g}$ be numbers satisfying (6), (6a), (cf. (4a)), (7), (7a) and also satisfying the italicized statement immediately following (7a). Let the coefficients in the series

$$
{ }_{k} \bar{\eta}_{w}(t)=\sum_{v=0}^{\infty}{ }_{h} \bar{\eta}_{w: v}^{j-1}, \quad(h=0, \cdots, j-1 ; w=1, \cdots, p)
$$

be defined by the equations ( $\overline{4})$, formed with the mentioned $\bar{c}$ and $\bar{g}$ and characterized in the italics preceding (6). In view of (8), whenever the series (17) all converge in a vicinity of $t=0$, the same will be true (in a possibly smaller vicinity of $t=0)$ for any set of formal solutions ${ }_{n} \tilde{\eta}_{w}(t)(c f .(3 \mathrm{a} ; \S 5))$ of the integral system $\left(\mathrm{A}_{\mathbf{8}}\right)$.

We are now in the position to form a system of integral equations

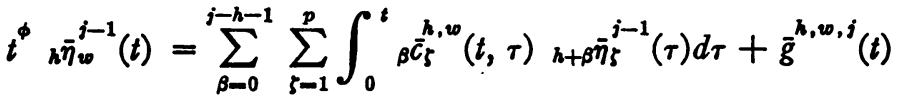

$$
\begin{aligned}
& (h=0, \cdots, j-1 ; w=1, \cdots, p) ;
\end{aligned}
$$

here

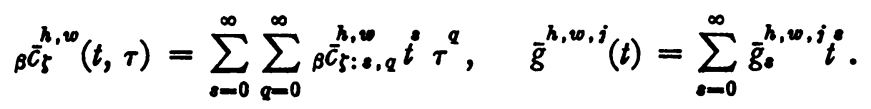

This system is satisfied by the set of formal series (17), defined by ( $\overline{4})$. Choose $c^{\prime}$ sufficiently small. Let $r$ be a sufficiently large positive number. Moreover, we shall choose

$$
\begin{array}{ll}
{ }_{\beta} \bar{c}_{\zeta}^{h, w}(t, \tau)=\sum_{i=\phi-1}^{\infty} r^{i} \sum_{H=0}^{i} t^{i-H}{ }_{\tau}^{H} & (\zeta \leqq w), \\
{ }_{\beta} \bar{c}_{\zeta}^{h, w}(t, \tau)=\sum_{i=\phi}^{\infty} r^{i} \sum_{H=0}^{i} t^{i-H}{ }_{\tau} B & (\zeta>w),
\end{array}
$$

except that

$$
{ }_{0} \bar{c}_{w}^{h, w}(t, \tau)=c^{\prime} r^{\phi-1} \sum_{H=0}^{\phi-1} t^{\phi-1-H} \tau^{H}+\sum_{i=\phi}^{\infty} r^{i} \sum_{H=0}^{i} t^{i-H} \tau^{H}
$$


These series, of course, all converge in the vicinity of $(t=0, \tau=0)$. It is seen that the constants, occurring in the second members of $(\overline{4})$, will then be

$$
r \bar{c}_{\zeta: \rho, u}^{h, w}=r^{\rho+u}, \quad \bar{g}_{Q+1}^{h, w}=r^{q-\phi+2},
$$

while

$$
{ }_{0} \bar{c}_{w: \phi-1-H, H}^{h, w}=c^{\prime} r^{\phi-1}=\bar{c} .
$$

The corresponding set of equations $(\overline{4})$ is not practicable for a proof of convergence of the solutions (17) of $\left(\bar{A}_{3}\right)$. Hence use will be made of a different set of relations, obtainable by utilizing the special form of $\left(\AA_{3}\right)$. In fact, (18a), (18b), (18c), (18d) are equivalent to

$$
\begin{array}{rlrl}
{ }_{\beta} \bar{c}_{\zeta}^{h, w}(t, \tau) & =\frac{1}{(1-r t)(1-r \tau)}-\sum_{\rho+u \leq \phi-2} r^{\rho+u} t^{\rho} \tau^{u} & (\zeta \leqq w), \\
{ }_{\beta} \bar{c}_{\zeta}^{h, w}(t, \tau) & =\frac{1}{(1-r t)(1-r \tau)}-\sum_{\rho+u \leq \phi-1} r^{\rho+u} t^{\rho} \tau^{u} & & (\zeta>w), \\
\bar{g}^{h, w, j}(t) & =r t^{\phi} /(1-r t), &
\end{array}
$$

except that

$$
{ }_{0} \bar{C}_{w}^{h, w}(t, \tau)=c^{\prime} r^{\phi-1} \sum_{H=0}^{\phi-1} t^{\phi-1-H} \tau^{H}+\frac{1}{(1-r t)(1-r \tau)}-\sum_{\rho+u \leqq_{\phi-1}} r^{p+u t p \tau^{u}} .
$$

Substitution of (20), (20a), (20b), (20c) in $\left(\bar{A}_{3}\right)$ results in

$$
\begin{aligned}
& \left(t^{\phi}-r t^{\phi+1}\right){ }_{h} \bar{\eta}_{w}^{j-1}(t)=\sum_{\beta=0}^{j-1-h} \sum_{\zeta=1}^{p} \int_{0}^{t} \frac{h+\beta \bar{\eta}_{\zeta}^{j-1}(\tau) d \tau}{1-r \tau} \\
& -(1-c) r^{\phi-1} \sum_{H=0}^{\phi-1}\left(t^{\phi-1-H}-r t^{\phi-H}\right) \int_{0}^{t} \tau^{H}{ }_{h{ }^{j}{ }^{j-1}}(\tau) d \tau \\
& -\sum_{\beta=0}^{j-1-h} \sum_{\zeta=1}^{p} \sum_{\rho+u \leq \phi-2}\left(r^{\rho+u} t^{\rho}-r^{\rho+u+1} t^{\rho+1}\right) \int_{0}^{t} \tau^{u}{ }_{h+\beta{ }^{\beta}}^{j-1}(\tau) d \tau \\
& -r^{\phi-1} \sum_{\beta=0}^{j-1-h} \sum_{\zeta=w+1}^{p} \sum_{u=0}^{\phi-1}\left(t^{\phi-1-u}-r t^{\phi-u}\right) \int_{0}^{t} \tau^{u}{ }_{h+\beta} \bar{\eta}_{\zeta}^{j-1}(\tau) d \tau+r t^{\phi} \\
& (h=0, \cdots, j-1 ; w=1, \cdots, p) .
\end{aligned}
$$

On using (17) the following formal relations are obtained:

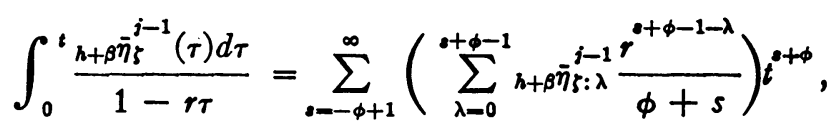




$$
\int_{0}^{t} \tau^{u}{ }_{h+\beta \bar{\eta}_{\zeta}^{j-1}}^{j}(\tau) d \tau=\sum_{s=0}^{\infty}{ }_{h+\beta \bar{\eta}_{\xi: \ell}^{j-1}} \frac{t^{s+u+1}}{s+u+1}
$$

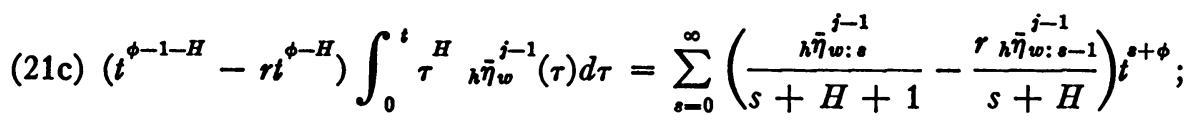

moreover, by virtue of the relationship

$$
\sum_{s=0}^{\infty} \sum_{i=0}^{\phi-2} a_{\theta, i} t^{\theta+i+1}=\sum_{--\phi+1}^{\infty} \sum_{i=0}^{\phi-2} a_{\phi+\ell-1-i, i} t^{\phi+,} \quad\left(\text { here } a_{\sigma, i}=0 \text { for } \sigma<0\right),
$$

it follows that

$$
\sum_{\rho+u \leq \phi-2}\left(r^{\rho+u} t^{\rho}-r^{p+u+1} t^{\rho+1}\right) \int_{0}^{t} \tau^{u}{ }_{h+\beta \eta^{j}}^{j-1}(\tau) d \tau
$$

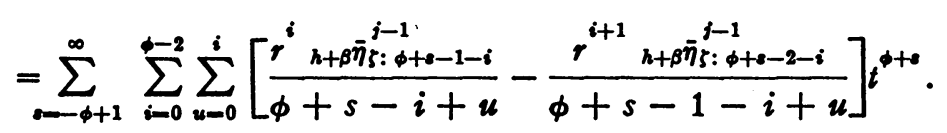

Substituting (21a), (21b), (21c), (21d) in (21) and observing that for $s<0$ the coefficient of $t^{\phi+\bullet}$ in the second member of (21d) may be written as

$$
\sum_{\lambda=0}^{+\phi+\phi} n+\beta \bar{\eta}_{s: \lambda}^{j-1} \frac{r^{\alpha+\phi-1-\lambda}}{\phi+s}
$$

and that for $s \geqq 0$ this coefficient may be expressed as

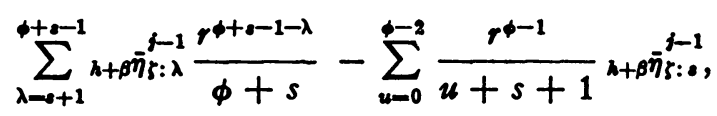

we obtain

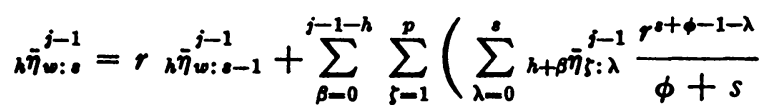

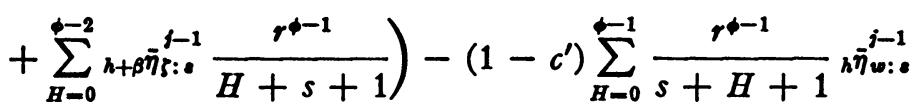

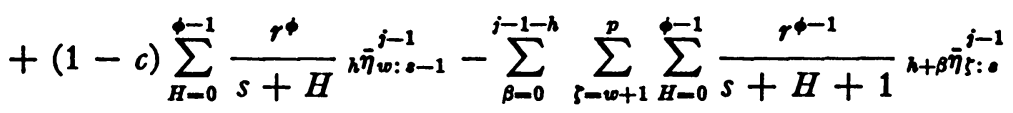

$$
\begin{aligned}
& +\sum_{\beta=0}^{j-1-h} \sum_{\zeta-\infty+1}^{p} \sum_{H=0}^{+-1} \frac{r^{\phi}}{s+B}{ }_{h+\beta \eta_{\zeta: s-1}^{j-1}}^{j-\xi(s) r} \\
& (s=0,1, \cdots ; h=1, \cdots, j-1 ; w=1, \cdots, p),
\end{aligned}
$$

where 


$$
\xi(s)=\left\{\begin{array}{l}
0 \\
1
\end{array}\right.
$$$$
(s=0) \text {. }
$$

On writing

$$
q_{s}=\sum_{H=0}^{\phi-1} \frac{r^{\phi-1}}{s+H+1}, \quad \bar{q}_{s}=\sum_{H=0}^{\phi} \frac{r^{\phi}}{s+H}, \quad g(s)=1-c^{\prime} q_{s},
$$

$c^{\prime}$ being small enough so that $g(s)>0$ for all $s>0$, and observing that in (22) all the ${ }_{h} \bar{\eta}_{\zeta \text { is }}^{j-1}(\zeta>w)$ cancel, we have

$$
\begin{aligned}
& g(s)_{h \bar{\eta}_{w: s}^{j-1}}^{j-1}=q \cdot \sum_{\zeta=1}^{w-1}{ }_{h} \bar{\eta}_{\zeta: s}^{j-1}+q \cdot \sum_{\beta=1}^{j-1-h} \sum_{\zeta=1}^{w}{ }_{h+\beta} \bar{\eta}_{\zeta: s}^{j-1} \\
& +\left(r g(s-1)+\bar{q}_{s-1}\right){ }_{h} \tilde{\eta}_{w: s-1}^{j-1}+\sum_{\beta=0}^{j-1-h} \sum_{\zeta=1}^{\infty} \frac{r}{\phi+s}{ }^{h+\beta \bar{\eta}_{\zeta: 0-1}^{j-1}}
\end{aligned}
$$

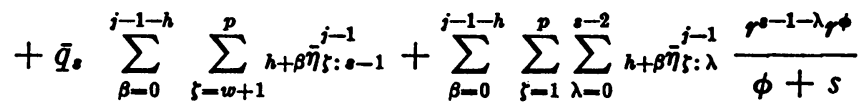

$$
\begin{aligned}
& +\xi(s) r \quad(s=0,1, \cdots ; h=0, \cdots, j-1 ; w=1, \cdots, p) .
\end{aligned}
$$

In view of (23) there exists a number $k$ so that

$$
\frac{1}{g(s)}, \quad\left(r g(s-1)+\bar{q}_{s-1}\right)<k,
$$

and such that, for $s \geqq 1$,

$$
q_{s}, \bar{q}_{s}, \frac{r \phi}{\phi+s}<\frac{k}{s} .
$$

Accordingly, the following inequalities are obtained from (24):

$$
\begin{aligned}
& h \bar{\eta}_{w: \ell}^{j-1}<k^{2}\left(\sum_{\zeta=1}^{w-1} h \bar{\eta}_{\zeta: 0}^{j-1}+\sum_{\beta=1}^{j-1-h} \sum_{\zeta=1}^{w} h+\beta \bar{\eta}_{\zeta: \ell}^{j-1}\right)
\end{aligned}
$$

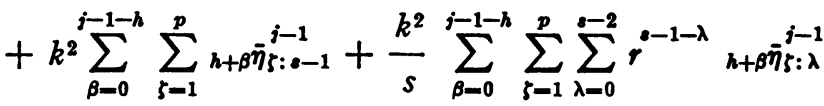

$$
\begin{aligned}
& (h=0, \cdots, j-1 ; w=1, \cdots, p ; s=1,2, \cdots) .
\end{aligned}
$$

With the aid of (26) convergence of the series (17) will be proved. For some positive $a$

$$
h^{i \eta_{w: 0}^{j-1}}<a \quad(h=0,1, \cdots, j-1 ; w=1, \cdots, p) .
$$

Let $s_{0}(\geqq 1)$ be a fixed integer. Depending on $s_{0}$, there exists a positive num- 
ber $\rho$, which will be taken $>r$, so that

$$
\left(l=0,1, \cdots, s_{0}-1 ; h=0,1, \cdots, j-1 ; w=1, \cdots, p\right) .
$$

By (26), formed for $s=s_{0}$, it will follow that

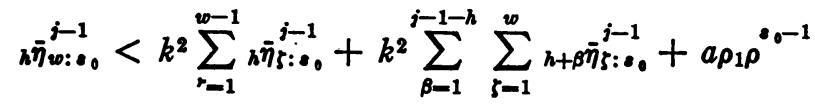

$$
\begin{aligned}
& (h=0,1, \cdots, j-1 ; w=1, \cdots, p),
\end{aligned}
$$

where $\rho_{1}=2 k^{2} \phi p$ and so is independent of $\rho$ and $s_{0}$. In particular, from (28) we have

$$
j-1 \bar{\eta}_{1: \Omega_{0}}^{j-1}<a \rho_{1} \rho^{\rho 0-1}
$$

Suppose now that

$$
j_{j-1}^{j-1} \bar{\eta}_{\zeta: 0_{0}}^{j-1}<a \rho_{w-1} \rho^{0,-1} \quad(\zeta=1, \cdots, w-1 ; 2 \leqq w \leqq p),
$$

where $\rho_{w-1}$ is independent of $\rho$ and $s$. From (28) it would then follow that

$$
j-1 \bar{\eta}_{w: s_{0}}^{j-1}<a \rho_{w \rho} \rho^{0-1}
$$

$$
\left(\rho_{w}=k^{2} p \rho_{w-1}+\rho_{1}\right) .
$$

Accordingly, by induction we have

$$
j-1 \bar{\eta}_{5: e_{0}}^{j-1}<a k_{1} \rho^{\bullet 0-1}
$$$$
(\zeta=1,2, \cdots \cdot p),
$$

where $k_{1}$ is independent of $\rho$ and $s$. Assume the truth of the following inequalities, reducing to (29) for $h=j-2$ :

$$
\begin{gathered}
\lambda \bar{\eta}_{\zeta: \rho_{0}}^{j-1}<a k_{j-h-1} \rho^{00-1} \\
(\lambda=j-1, j-2, \cdots, h+1 ; \zeta=1, \cdots, p ; 0 \leqq h \leqq j-2),
\end{gathered}
$$

where $k_{j-h-1}$ is independent of $\rho$ and $s_{0}$. From (28) we then find that

$$
{ }_{h} \bar{\eta}_{1: \rho_{0}}^{j-1}<a k_{j-h-1,1} \rho^{8,-1},
$$

where $k_{j-h-1,1}=k^{2} \phi p k_{j-h-1}+\rho_{1}$ so that $k_{j-h-1,1}$ is independent of $\rho, s_{0}$. It is furthermore noted that the inequalities

$$
h_{\eta=\varepsilon_{0}}^{j-1}<a k_{j-h-1, w-1} \rho^{s,-1} \quad(\zeta=1, \cdots, w-1 ; 2 \leqq w \leqq p),
$$

where $k_{j-h-1, w-1}$ is assumed independent of $\rho$ and $s_{0}$, together with (30) would imply, by virtue of (28), that 


$$
\begin{gathered}
h_{\eta_{w: s,}^{j-1}<a k_{j-h-1, w} \rho^{80-1},}, \\
k_{j-h-1, w}=k^{2} p k_{j-h-1, w-1}+\phi \phi k_{j-h-1}+\rho_{1} .
\end{gathered}
$$

Thus,

$$
h \bar{\eta}_{\zeta: s_{0}}^{j-1}<a k_{j-h} \rho^{\rho_{0}-1} \quad(\zeta=1, \cdots, p),
$$

$k_{j-h}$ being independent of $\rho$ and $s_{0}$. The inequalities (30) imply (32); whence we conclude that for some $k$, independent of $\rho$ and $s_{0}$,

$$
\lambda \bar{\eta}_{\zeta: \Omega_{0}}^{j-1}<a \bar{k} \rho_{\rho}^{80-1} \quad(\lambda=j-1, j-2, \cdots, 0 ; \zeta=1, \cdots, p) .^{*}
$$

Choose the number $\rho$, first introduced in (27a), so that $\rho \geqq k$. In view of (33) it is then observed that (27a) holds not only for $\left(l=0, \cdots, s_{0}-1 ; h=0\right.$, $1, \cdots, j-1 ; w=1, \cdots, p)$, as originally stated, but necessarily also for $\left(l=s_{0} ; h=0, \cdots, j-1 ; w=1, \cdots, p\right)$. Since inequalities (28) remain valid, when $s_{0}$ is replaced by any positive integer $s$, provided only that

$$
(l=0,1, \cdots, s-1 ; h=0, \cdots, j-1 ; w=1, \cdots, p),
$$

it follows that (34) is valid for $(l=0,1, \cdots ; h=0, \cdots, j-1 ; w=1, \cdots, p)$.

By virtue of (34) the formal solutions (17) of the dominant system of integral equations $\left(\bar{A}_{3}\right)$ all converge for $|t|<1 / \rho$.

7. The main theorem for differential equations. On taking account of the two italicized statements, one at the very end of $\$ 6$ and the other preceding $\left(\bar{A}_{3}\right)(\S 6)$, the truth of the following lemma becomes evident.

LEMMA 4. Suppose that a system of integral equations $\left(\mathrm{A}_{3}\right)(c f . \S 5)$ is given with the following properties:

(i) The coefficients of the system are series of the form $(21 ; \S 5),(21 \mathrm{a} ; \S 5)$ convergent for $|t|<\rho^{\prime},|\tau|<\rho^{\prime}$.

(ii) $(23$; §5) is satisfied.

(iii) The system possesses a full set of formal series solutions ${ }_{h} \tilde{\eta}_{w w}^{j-1}(t)$ of the form $(3 \mathrm{a} ; \S 5)$.

Under these conditions, the elements of any set of formal solutions, referred to in (iii), will all necessarily converge in some vicinity of the origin.

It is to be noted that this lemma makes no reference to properties of the coefficients of $\left(\mathrm{A}_{3}\right)$ at $(t=\infty, \tau=\infty)$; it also makes no reference to the manner in which such a system might have originated. In view of Lemma 3 , it is

$* \bar{k}$, in general, depends on $r$. 
observed that Lemma 4 is applicable to the particular system $\left(A_{3}\right)$, established in \$5. The formal solutions of the latter system, therefore, all converge in some neighborhood of the origin. We shall now prove the following Lemma, referring to properties at infinity of the analytic continuations of the analytic functions so defined near $t=0$.

LEMMA 5. Suppose that a system of integral equations $\left(\mathrm{A}_{3}\right)$ is given which satisfies the conditions of Lemma 3 . The elements ${ }_{h} \tilde{\eta}_{w v}^{j-1}(t)(h=0, \cdots, j-1$; $w=1, \cdots, p$ ) belonging to any particular set of solutions of $\left(\mathrm{A}_{3}\right)$ (as follows by Lemma 4 , they are necessarily analytic at $t=0$ ) have analytic continuations in $P_{0}$ (cf. italics preceding $(24 ; \S 5)$ ) for which

$$
\left|{ }_{h} \tilde{\eta}_{w}^{j-1}(t)\right|<C e^{e|t|} \quad(h=0,1, \cdots, j-1 ; w=1, \cdots, p),
$$

along every ray $\bar{t}(=\angle t)$ in $P_{0}$. In (1) $C$ and $q$ are positive, sufficiently great ${ }_{1}$ and are independent of $\bar{t}$ and $|t|$.

The proof of this Lemma will be somewhat similar to that which J. Horn gives in proving certain inequalities analogous to (1). We confine ourselves to some particular set of solutions of $\left(\mathrm{A}_{3}\right) . C$ will be chosen a fixed value such that

$$
\left|{ }_{h} \tilde{\eta}_{w: 0}^{j-1}\right|<C \quad(h=0,1, \cdots, j-1 ; w=1, \cdots, p) .
$$

Then

$$
\left|{ }_{h} \tilde{\eta}_{w}^{j-1}(t)\right|<C e^{q|t|}
$$$$
(h=0, \cdots, j-1 ; w=1, \cdots, p)
$$

for $|t| \leqq r^{0}\left(r^{0}\right.$ some positive number) and for every $q \geqq 0$. It will be supposed that the constant $\bar{\rho}$, occurring in $(25 ; \S 5)$, had been chosen suitably small and so that

$$
0<\bar{\rho}<r^{0} .
$$

Suppose the Lemma is not true. Then, for some $r^{\prime}>r^{0}$, the inequalities

$$
\left|{ }_{h}^{j-j}(t)\right|<C e^{q|\theta|} \quad(h=0, \cdots, j-1 ; w=1, \cdots, p)
$$

will be valid for $|t|<r^{\prime}$ along every ray $\bar{t}(=\angle t)$ in $P$ (cf. definition following $(15 \mathrm{a} ; \S 5)$ ), while, for some $h=h^{\prime}$ and $w=w^{\prime}$, we shall have

$$
\left|h_{h^{\prime}} \tilde{\eta}_{w^{\prime}}^{j-1}(t)\right|=C e^{q|t|}
$$

$$
\left(t=t^{\prime}=r^{\prime} e^{i \bar{t}^{\prime}} ; \bar{t}^{\prime} \text { in } P\right) .
$$

It is clear that (4) continues to hold, with $<$ replaced by $\leqq$, when $t$ is on any ray $\bar{t}$ of $P_{0}$, while $|t| \leqq r^{\prime}$. Let the integrations involved below be along the 
ray $\bar{t}^{\prime}$. From $\left(\mathrm{A}_{3}\right)(\S 5)$, by virtue of $(4),(4 a)$ and $(25 a ; \S 5)$, it then follows that

$$
\left|t^{\prime \phi}{ }_{n}^{\prime} \tilde{\eta}_{w^{\prime}}^{j-1}(t)\right|=\left|t^{\prime}\right|^{\phi} C e^{q\left|t^{\prime}\right|}
$$

$$
<\sum_{\beta=0}^{j-h-1} \sum_{\zeta=1}^{p} \int_{0}^{r^{\prime}}\left|{ }_{\beta}^{* h C_{\zeta}^{h, w}}\left(t^{\prime}, \tau\right)\right| C e^{q|\tau|} d|\tau|+R_{0}\left|t^{\prime}\right|^{\phi}\left|t^{\prime}\right|^{-n} e^{\rho\left|t^{\prime}\right|} .
$$

It is observed that

$$
\sum_{\beta=0}^{j-h-1} \sum_{\zeta=1}^{p} \int_{0}^{\bar{\rho}}\left|{ }_{\beta}^{* h, w}\left(t^{\prime}, \tau\right)\right| C e^{q|\tau|} d|\tau|<\bar{l} e^{\alpha \bar{\rho}},
$$

where $\bar{l}$ is independent of $t^{\prime}$ and $q$. Thus from (5), on using (25; §5), we obtain the inequality

$$
\begin{aligned}
&\left(r^{\prime}\right) \phi C e^{q r^{\prime}}<l e^{q \bar{\rho}}+R_{0}\left(r^{\prime}\right)^{\phi-n} e^{\rho r^{\prime}} \\
&+\sum_{\beta=0}^{j-h-1} \sum_{\zeta=1}^{p} \int_{\bar{\rho}}^{r^{\prime}} R_{0}\left(r^{\prime}\right) \phi e^{\rho\left(r^{\prime}-|\tau|\right) C e^{q|\tau|} d|\tau| ;}
\end{aligned}
$$

whence

(6a)

$$
1<\frac{1}{C} i e^{-q\left(r^{\prime}-\bar{\rho}\right)}\left(r^{\prime}\right)^{-\phi}+\frac{1}{C} R_{0}\left(r^{\prime}\right)^{-n} e^{-(q-\rho) r^{\prime}}
$$

$$
+R_{0} p \phi \int_{\bar{p}}^{r^{\prime}} e^{-(q-p)\left(r^{\prime}-|\tau|\right)} d|\tau| .
$$

Take $q>\rho$. It is then concluded that the integral in the second member of (6a) satisfies the inequality

$$
\int_{\bar{p}}^{r^{\prime}}<\left[\int_{0}^{r^{\prime}}=\int_{0}^{r^{\prime}} e^{-(q-\rho) x} d x<\int_{0}^{\infty} e^{-(q-\rho) x} d x=\right] \frac{1}{q-\rho} .
$$

Since $r^{\prime}>r_{0}>\bar{\rho}$, in view of (6b) from (6a) we obtain

$$
1<\frac{1}{C} \bar{l}_{e^{-q\left(r^{0}-\bar{p}\right)}\left(r^{0}\right)-\phi}+\frac{1}{C} R_{0}\left(r^{0}\right)^{-n} e^{-(q-\rho) r^{0}}+\frac{R_{0} p \phi}{q-\rho}=f(q) .
$$

Now, $r^{0}$ is chosen independent of $q$. It is further noted that $f(q)$ is continuous in $q$, for $q>\bar{\rho}$; moreover,

$$
\lim _{q \rightarrow \infty} f(q)=0 .
$$

Accordingly, $q(>\rho)$ may be taken sufficiently great so that $f(q) \leqq 1$; for any such value of $q$ a contradiction arises to (7). Therefore the Lemma is seen 
to be true. The inequalities (1) of this Lemma will hold, for instance, when $q$ is such that $f(q)=1$.

Consider a particular ray $\bar{t}$ in $P$. The formal series ${ }_{h} \eta_{w^{j-1}}(x)$ (cf. $\left.(3 ; \S 5)\right)$, even if divergent, give rise to certain analytic functions

$$
{ }_{h \eta_{w}}^{j-1}(x)=\int_{0}^{\infty}{ }_{h} \tilde{\eta}_{w}^{j-1}(t) e^{t x} d t \quad(h=0, \cdots, j-1 ; w=1, \cdots, p) .
$$

The truth of this statement is a consequence of the following considerations. Suppose $x$ is in a half plane $H[\bar{t}]$, characterised by an inequality of the form

$$
R\left(e^{i \bar{i}} x\right)=|x| \cos (\bar{t}+\bar{x})<-q^{\prime}(<0) \quad(\bar{x}=\angle x) ;
$$

for the present we shall take $q^{\prime}=q+\epsilon(\epsilon>0$ and arbitrarily small). It then follows that the integrals (8) are all absolutely convergent. In fact, by (1) and (9),

$$
\begin{aligned}
\left|{ }_{k \eta_{w}^{j}}^{j-1}(x)\right| \leqq & \int_{0}^{\infty}\left|h \tilde{\eta}_{w}^{j-1}(t)\right| \exp (|t||x| \cos (\bar{t}+\bar{x})) d|t| \\
< & <\int_{0}^{\infty} e^{-\epsilon|t|} d|t|=\frac{C}{\epsilon} \\
& (x \text { in } H[\bar{t}] ; h=0 ; \cdots, j-1 ; w=1, \cdots, p) .
\end{aligned}
$$

The conditions $(1 ; \S 5),(5 ; \S 5)$ have to be satisfied. The first of these is seen to hold for $x$ in $H[\bar{t}]$ since, by virtue of (9),

$$
\left|e^{t x t^{\alpha}}\right|<e^{-(a+e)|t|}|t|^{\alpha} \text {. }
$$

On the other hand, the following inequalities will hold for the functions within the brackets of $(5 ; \S 5)$ :

$$
\begin{aligned}
\left|e^{t x} \int_{0}^{t}\right| & <C e^{-(q+e)|t|} \int_{0}^{|t|}|\tau| k e^{q|\tau|} d|\tau|^{(B)} \\
<C e^{-\epsilon|t|} \int_{0}^{|t|}|\tau|^{k} d|\tau|^{(B)} & =\frac{C e^{-\epsilon|t|}|t|^{k+H}}{(k+1) \cdots(k+H)} \\
& (H=1,2, \cdots ; k=0, \cdots, n) .
\end{aligned}
$$

Since the last expression above vanishes for $t=0$ and approaches zero as $|t| \rightarrow \infty$, the truth of $(5 ; \$ 5)$ becomes evident.

Thus, the conditions of certain theorems, due to N. E. Nörlund and of central importance in the theory of factorial series, ${ }^{*}$ are seen to be valid, a fact made manifest after certain obvious transformations are carried out. We thus obtain, whenever the ray $\angle t=\bar{t}$ is in $P$,

* N. E. Nörlund, Legons sur les Séries d'Interpolation, Paris, 1926; pp. 206-208. Also see his theorem on p. 203. 


$$
\begin{aligned}
{ }_{h} \eta_{w}^{j-1}(x)= & \sum_{s=0}^{\infty} \frac{{ }_{h} a_{w: s}^{j-1}(\bar{t})}{x(x-\gamma) \cdots(x-s \gamma)} \\
& (h=0, \cdots, j-1 ; w=1, \cdots, p ; \angle \gamma=\bar{\gamma})
\end{aligned}
$$

where $|\gamma|$ is suitably great, $\bar{\gamma}=-\bar{t}$, and the involved series all converge in a certain half plane $B[\bar{t}]$. These functions are analytic solutions of the system of "mixed" differential equations $\left(\mathrm{A}_{2}\right)(\$ 4)$. It is clear that results of stated type hold for $j=1, \cdots, \phi$. Accordingly, it is observed that the $\phi$ formal series solutions of $\left(\mathrm{A}_{1}\right), s_{j}(x)$ (cf. $\left.(1 ; \S 3),(1 \mathrm{~b} ; \S 3)\right)$, give rise to a corresponding set of $\phi$ analytic solutions $y_{j}(x)$, obtained by replacing the ${ }_{h} \eta_{w}{ }^{j-1}(x)$ of $(1 ; \S 3)$ by the corresponding (convergent) series (10). We are thus ready to formulate the Main Theorem for differential equations.

THEOREM I. Let a differential equation (A) (\$1) be given. Suppose that corresponding to a root $\rho_{1}$, of multiplicity $\phi$, of the associated characteristic equation there exists a linearly independent set of $\phi$ formal series solutions, all of normal type (cf. \$2) and all forming one logarithmic group. Bring (A) to the corresponding form $\left(\mathrm{A}_{1}\right)(\$ 2)$ and let $E(\rho)=0$ be the characteristic equation of $\left(\mathrm{A}_{1}\right)$. For every $\bar{t}$, not coincident with a value of an angle of a non-zero root of $E(\rho)=0$,* the following is true.

$\left(A_{1}\right)$ possesses a set of $\phi$ linearly independent analytic solutions

$$
\begin{array}{r}
y_{j}(x)=e^{Q(x)} x \sum_{h=0}^{r-1} \log ^{h} x\left({ }_{h \eta_{0}^{j-1}}^{j-1}+\sum_{w=1}^{p} x^{(p-w) / p} \sum_{i=0}^{\infty} \frac{{ }_{h} a_{w: s}^{j-1}(\bar{t})}{x(x-\gamma) \cdots(x-s \gamma)}\right) \\
\left(j=1, \cdots, \phi ; \quad \angle \gamma=\bar{\gamma}=-\bar{t} ; \quad Q(x) \text { a polynomial in } x^{1 / p}\right),
\end{array}
$$

where $|\gamma|$ is suitably great and the involved series all converge in a certain half plane $B[\bar{t}]$ (cf. (9); $q^{\prime}$ in (9) sufficiently great).

The implications of this theorem for (A) are immediate. The $\phi$ corresponding solutions of (A) will be of the form (11), where $x$ is replaced by a certain power of $x$; accordingly, the series involved in these solutions will all converge in certain sectors. $\dagger$

The theorem is of greatest possible completeness in the sense that even normal formal series solutions of (A) do not always lead to convergent factorial series developments, if corresponding to the multiple root, in question, there

\footnotetext{
* It is supposed, as may be without any loss of generality, that $\rho=0$ is the root corresponding to the formal solutions in question.

$\dagger$ This is a consequence of known properties of factorial series.
} 
is more than one logarithmic group. Consideration of the following example will demonstrate this statement.

The equation

$$
L_{3}(y) \equiv y^{(3)}(x)+a x^{-1} y^{(1)}(x)+d x^{-3} y(x)=0 \quad(a \neq 0, d \neq 0)
$$

is of the form $\left(A_{1}\right)$; its characteristic equation has a root, $\rho=0$, of multiplicity three. Moreover, it has a formal solution

$$
y(x)=\sum_{v=0}^{\infty} y_{\nu} x^{-\nu} \quad\left(y_{0}=1\right) .
$$

In fact, substituting (13) in (12), we obtain

$$
-L_{3}(y) \equiv \sum_{\lambda=0}^{\infty} f_{\lambda} x^{-\lambda-3}, \quad f_{\lambda}=[\lambda(\lambda+1)(\lambda+2)-d] y_{\lambda}+a(\lambda+1) y_{\lambda+1}
$$

so that the equations $f_{\lambda}=0(\lambda=0,1, \cdots)$ are seen to be uniquely solvable for the $y_{v}$. We have

$$
y_{\lambda+1}=q(\lambda) y_{\lambda}, \quad q(\lambda)=\frac{d}{a(\lambda+1)}-\frac{\lambda(\lambda+2)}{a} \quad(\lambda=0,1, \cdots) ;
$$

whence

$$
y_{\lambda+1}=q(0) q(1) \cdots q(\lambda) \quad(\lambda=0,1, \cdots) .
$$

It can be shown that the number of logarithmic groups is greater than one.

Suppose that corresponding to the normal solution of (12) there is some convergent factorial series development, of the form

$$
1+\sum_{s=0}^{\infty} \frac{a}{x(x-\gamma) \cdots(x-s \gamma)}
$$

where $\gamma$ is some real or complex number. By a theorem of Nörlund referred to previously, this function would be expressible by means of the convergent integral

$$
\eta(x)=1+\int_{0}^{\infty} \tilde{y}(t) e^{t x} d t
$$

where the integration is extended along a certain ray and where $\tilde{y}(t)=\sum_{\lambda-0}^{\infty} \tilde{y}_{\lambda} t^{\lambda}$ is analytic at $t=0$. Since $\eta(x)$ satisfies (12) it would necessarily follow that

$$
\tilde{y}_{\lambda}=\frac{(-1)^{\lambda+1} y_{\lambda+1}}{\lambda !} \quad(\lambda=0,1, \cdots),
$$


the $y_{\lambda+1}$ being defined by (14a). Accordingly,

$$
\left|\frac{\tilde{y}_{\lambda}}{\tilde{y}_{\lambda-1}}\right|=\left|\frac{q(\lambda)}{\lambda}\right|=\left|\frac{d}{a \lambda(\lambda+1)}-\frac{\lambda+2}{a}\right|
$$

so that $\left|\tilde{y}_{\lambda} / \tilde{y}_{\lambda-1}\right| \rightarrow \infty$, as $\lambda \rightarrow \infty$. Thus, a contradiction arises to analyticity of $\tilde{y}(t)$. Hence there exists no convergent factorial series corresponding to (13).

\section{PART II. LiNeAR DIFFERENCE EQUATIONS}

8. Some preliminary facts concerning difference equations. As had been demonstrated by Birkhoff, the difference equation (B) ( $\$ 1$ ) possesses in all cases a linearly independent set of $n$ formal series solutions

$$
s_{i}(x)=e^{\mu_{i} x \log x} e^{\ell_{i}(x)} x^{r_{i} \sigma_{i}}(x) \quad(i=1, \cdots, n),
$$

where

$$
\begin{aligned}
& Q_{i}(x)=\sum_{v=0}^{k_{i}-1} q_{v}^{i} x^{\left(k_{i}-v\right) / k_{i}}, \\
& \sigma_{i}(x)=\sum_{h=0}^{m_{i^{\prime}}} \log ^{h} x_{h} \eta^{m_{i^{\prime}}(x),} \\
& { }_{n} \eta^{m_{i^{\prime}}}(x)=\sum_{s=0}^{\infty}{ }_{h} \eta_{s}{ }^{m_{i^{\prime}}} x^{-s / k_{i}}
\end{aligned}
$$$$
\left(h=0, \cdots, m_{i}\right)
$$

here the $\mu_{i}$ are certain rational numbers, the $m_{i}^{\prime}$ and $k_{i}$ are integers $\left(m_{i}^{\prime} \geqq 0\right.$; $k_{i}=r_{i}^{\prime} p$; integer $r_{i}^{\prime} \geqq 1$ ). The formal series can be arranged in logarithmic groups, the exponential factor

$$
e^{\mu x \log x} e^{Q(x)} x^{r}
$$

being the same for each member of the same group.* It is convenient to group the $\mu_{i}$ as follows:

$$
\begin{array}{rr}
m_{1}=\mu_{1}=\mu_{2}=\cdots=\mu_{\alpha_{1}}>m_{2}=\mu_{\alpha_{1}+1}=\mu_{\alpha_{1}+2}=\cdots=\mu_{\alpha_{2}}>\cdots \\
>m_{\lambda}=\mu_{\alpha_{\lambda-1}+1}=\mu_{\alpha_{\lambda-1}+2}=\cdots=\mu_{\alpha_{\lambda}}\left(=\mu_{n}\right) & (\lambda \geqq 1) .
\end{array}
$$

It is to be noted that the formal facts continue to hold when the $d_{i, j}(x)$ are divergent series. To each one of the $\lambda \mu$-groups, specified in (2), there corresponds a certain characteristic equation.

For the case when the coefficients of (B), except for a few positive integral powers of $x$, are expressible by convergent factorial series of the type

$$
\sum_{s=0}^{\infty} \frac{a_{s}}{x(x-\gamma) \cdots(x-\gamma s)} \quad(\gamma=1 \text { or }-1)
$$

* The definition for such groups is analogous to a similar definition in $\$ 2$. 
and, besides, (B) can be brought to the form of an equation of "Fuchsian type," Nörlund obtains full sets of solutions, which are expressible by means of convergent factorial series of the form (3), ${ }^{*}$ thus establishing an analogy to the Fuchsian theory in the field of differential equations.

Nörlund also treats the class of equations (B) where the coefficients can be brought to the form of polynomials in $x$. His other restrictions on the coefficients amount precisely to the following.

(i) There is only one $\mu$-group.

(ii) All the numbers $\mu_{i}$ are zero.

(iii) There exist no anormal formal series solutions. $\dagger$

On using Laplace transformations, leading to ordinary linear differential equations, he obtains full sets of solutions expressible with the aid of convergent factorial series (3), where $\gamma$ may be complex and $|\gamma|$ is sufficiently great. $\ddagger$

In Horn's work we essentially have an equation (B), whose coefficients contain no fractional powers of $x$, and which has only one $\mu$-group, all the $\mu_{i}$ being zero. Moreover, he assumes that all the roots of the characteristic equation (necessarily, there will be only one such equation) are distinct. Under these conditions, he obtains solutions with the aid of convergent factorial series (3) ( $|\gamma|$ sufficiently great; certain values of $\angle \gamma$ excepted).

Contrary to the restrictions of Nörlund's and Horn's works, in the present paper existence of several $\mu$-groups (cf. (2)) is admitted; moreover, the coefficients of (B) are allowed to contain fractional powers of $x$.

Consider any particular $\mu$-group. The $\mu_{i}$ of this group will all have the same value, say $\mu_{1}$. By means of a transformation of the form

$$
y(x)=e^{\mu x \log x} z(x)
$$

(B) will be brought to the form $\left(B_{1}\right)$. Retaining the notation originally used in (B) (cf. \$1) the new equation will be considered as the equation (B), whose coefficients are convergent series

$$
d_{n-k}(x)=\sum_{s=0}^{\infty} d_{n-k, s} x^{-s / p} \quad(k=0,1, \cdots, n), \S
$$

where

* Cf. Nörlund on difference equations, loc. cit., pp. 28-56; in these pages he also establishes certain other important results.

† That is, the $Q_{i}(x)$ and the $\sigma_{i}(x)$ (cf. (1), (1a), (1b), (1c)) contain no fractional powers of $x$; on the other hand, logarithms in the formal series may be present.

$\ddagger$ Cf. Nörlund on difference equations, loc. cit., pp. 56-88.

$\S$ In (4), $p$ will be, in general, different from the corresponding integer in (B). 


$$
\begin{aligned}
& d_{0,0}=d_{1,0}=\cdots=d_{m-1,0}=d_{m+H+1,0}=d_{m+H+2,0}=\cdots=d_{n, 0}=0 \text {, } \\
& d_{m, 0}, \quad d_{m+H, 0} \neq 0 \quad(0 \leqq m ; m \leqq m+H \leqq n) .
\end{aligned}
$$

If the $\mu$-group under consideration is the one corresponding to a greatest $\mu_{i}$, the equation $\left(\mathrm{B}_{1}\right)$ will have coefficients for which (4a), with $m=0$, will hold.* To the $\mu$-group, under consideration, of (B) there corresponds a certain $\mu$ group of $\left(B_{1}\right)$; the $\mu_{i}$ of the latter group will be all zero. A formal solution (with $\mu=0$ ) of $\left(B_{1}\right)$ is said to be normal when the corresponding polynomial $Q(x)$ (1a) and the corresponding series $\sigma(x)((1 \mathrm{~b}),(1 \mathrm{c}))$ are in integral powers $x^{1 / p}$. In the contrary case, that is, when $Q(x)$ and $\sigma(x)$ are actually in integral powers of $x^{1 /\left(r^{\prime} p\right)}$ (integer $r^{\prime}>1$ ), the formal series of $\left(B_{1}\right)$ is termed anormal. A formal solution of (B), with $\mu$ not necessarily zero, is normal or anormal according as the corresponding solution (with $\mu=0$ ) of $\left(B_{1}\right)$ is normal or anormal.

Throughout the text, leading up to the Main Theorem for difference equations (\$12), we consider a root $\rho^{\prime}$ of multiplicity $\phi$ of the characteristic equation, of (B), associated with a particular $\mu$-group. The only restriction, and as will be shown by an example in $\$ 12$ a necessary one, will be that associated with this root $\rho^{\prime}$ there is just one logarithmic group and that all the formal solutions in this group are normal. When, as will be done for convenience, the corresponding equation $\left(B_{1}\right)((4),(4 a))$ is used, the characteristic equation at hand of $\left(B_{1}\right)$ will be

$$
E_{1}(\rho)=\sum_{k} d_{n-k, 0} e^{k \rho}=0 .
$$

This equation will possess a root of $\rho=\rho_{1}$ of multiplicity $\phi$.

9. Conditions for existence of formal solutions of the type specified in $\S 8$. A set of $\phi$ linearly independent normal formal series solutions (of $\left(B_{1}\right)$ ), which by hypothesis corresponds to the root $\rho=\rho_{1}$ of $(5 ; \S 8)$, forms a logarithmic group. These solutions will be written in the form

$$
s_{j}(x)=e^{Q(x)} x^{r} \sum_{h=0}^{j-1} \log ^{h} x_{h} \eta^{j-1}(x) \quad(j=1, \cdots, \phi),
$$

where

$$
{ }_{h} \eta^{j-1}(x)=\sum_{s=0}^{\infty}{ }_{h} \eta_{s}^{j-1} x^{-s / p} \quad(h=0,1, \cdots, j-1) .
$$

Since, in (1), $Q(x)$ is in powers of $x^{1 / p}$ it follows that the transformation

$$
y(x)=e^{Q(x)} x^{r} \bar{y}(x),
$$

* If there exists only one $\mu$-group, $m=0$ and $m+B=n$. 
applied to $\left(B_{1}\right)$, does not change the form of $\left(B_{1}\right)$; in particular, the coefficients of the transformed equation will also be in powers of $x^{1 / p}$. Thus, without loss of generality, it will be assumed that

$$
e^{Q(x)} x^{r} \equiv 1
$$

and that not all the

$$
j_{-1}^{j} \eta_{0}^{j-1}
$$$$
(j=1, \cdots, \phi)
$$

are zero. We shall presently find the conditions satisfied by the coefficients of $\left(B_{1}\right)$, when this difference equation possesses solutions (1), (1a), for which (1b) holds.

Given a series

$$
\eta(x)=\sum_{s=0}^{\infty} \eta_{0} x^{-s / p}
$$

we shall have

$$
\begin{aligned}
\eta(x+k) & =\sum_{s=0}^{\infty} \eta_{s} x^{-s / p} \sum_{r=0}^{\infty} C_{r}^{-s / p} k^{r} x^{-r} \\
& =\eta_{0}+\sum_{r=0}^{\infty} \sum_{v=0}^{\infty} \sum_{w=1}^{p} C_{r}^{-(p p+w) / p} k^{r} \eta_{p p+w} x^{-((r+v) p+w) / p} \\
& =\eta_{0}[k]+\sum_{\lambda=0}^{\infty} \sum_{w=1}^{p} \eta_{\lambda p+w}[k] x^{-(\lambda p+w) / p}
\end{aligned}
$$

where $\eta_{0}(k)=\eta_{0}$ and

$$
\eta_{\lambda p+w}[k]=\sum_{p=0}^{\lambda} C_{\lambda \rightarrow p}^{-(v p+w) / p} k^{\lambda-\nu} \eta_{p p+w} \quad(\lambda=0,1, \cdots ; w=1, \cdots, p) .
$$

A certain expression for $\log ^{h}(x+k)$ will also be needed. It is observed that

$$
\log (x+k)=\log x+\sum_{\alpha=1}^{\infty} \frac{(-1)^{\alpha+1}}{\alpha} k^{\alpha} x^{-\alpha} .
$$

Moreover, it can be shown without difficulty that

$$
\left(\sum_{\alpha=1}^{\infty} \frac{(-1)^{\alpha+1}}{\alpha} k^{\alpha} x^{-\alpha}\right)^{\beta}=\sum_{\alpha=0}^{\infty}(-1)^{\alpha+\beta} k^{\alpha} l_{\alpha, \beta} x^{-\alpha},
$$

where

$$
l_{0,0}=1, \quad l_{\alpha, 0}=0 \quad(\alpha \geqq 1), \quad l_{\alpha, \beta}=0 \quad(\alpha<\beta)
$$


the $l_{\alpha, \beta}$ not mentioned in (3a) are all positive. We then have

$$
\log ^{h}(x+k)=\sum_{\beta=0}^{h} \log ^{h-\beta} x C_{\beta}^{h} \sum_{\alpha=0}^{\infty}(-1)^{\alpha+\beta} k^{\alpha} l_{\alpha, \beta} x^{-\alpha} .
$$

By (2), (2a), (3b), on making use of the identity

$$
\sum_{h=0}^{j-1} \sum_{\beta=0}^{h} a_{h-\beta} b_{h, \beta}=\sum_{h=0}^{j-1} a_{h} \sum_{\beta=0}^{j-h-1} b_{h+\beta, \beta}
$$

it follows that, formally,

(4) $s_{j}(x+k)=\sum_{h=0}^{j-1} \log ^{h} x \sum_{\beta=0}^{j-h-1} \sum_{\alpha=0}^{\infty} C_{\beta}^{h+\beta}(-1)^{\alpha+\beta} k^{\alpha} l_{\alpha, \beta} x^{-\alpha} \sum_{\ell=0}^{\infty}{ }_{h+\beta \eta_{\ell}}^{j-1}[k] x^{-z / p}$.

On substituting $(4)$ in $\left(B_{1}\right)$ and on noting $(4 ; \S 8)$, it is observed that

$$
L\left(s_{j}\right)=\sum_{k} d_{n-k}(x) s_{j}(x+k)=\sum_{h=0}^{i-1} \log ^{h} x_{h} f^{j-1}(x),
$$

where

$$
\begin{aligned}
{ }_{h} f^{j-1}(x) & =\sum_{\alpha=0}^{\infty} \sum_{s=0}^{\infty} \sum_{\sigma=0}^{\infty} a_{\alpha, 8, \sigma}^{j, h} x^{-(\alpha+(s+\sigma) / p)}, \\
a_{\alpha, 8, \sigma}^{i, h} & =\sum_{\beta=0}^{j-h-1} \sum_{k=0}^{n} a_{\alpha, s, \sigma, \beta, k}^{j, h}, \\
\alpha_{\alpha, s, \sigma, \beta, k}^{j, h} & =C_{\beta}^{h+\beta}(-1)^{\alpha+\beta} l_{\alpha, \beta, h+\beta \eta_{s}}^{j-1}[k] k^{\alpha} d_{n-k, \sigma}
\end{aligned}
$$

Further examination of ${ }_{h} f^{i-1}(x)$ gives

$$
\begin{aligned}
h f^{j-1}(x) & =\sum_{\alpha=0}^{\infty} \sum_{\tau=0}^{\infty} a_{\alpha, \tau}^{j, h} x^{-(\alpha p+\tau) / p}, \\
a_{\alpha, \tau}^{j, h} & =\sum_{s=0}^{\tau} a_{\alpha, s, \tau-s .}^{j, h}
\end{aligned}
$$

It follows next that

$$
{ }_{h} f^{j-1}(x)=\sum_{\alpha=0}^{\infty} \sum_{u=0}^{\infty} \sum_{v=0}^{p-1} a_{\alpha, u p+v}^{j, \lambda} x^{-((\alpha+u) p+v) / p}=\sum_{p=0}^{\infty} \sum_{r=0}^{p-1}{ }_{h} F_{p, r}^{j-1} x^{-(p p+\tau) / p},
$$

where

$$
{ }_{h} F_{\rho, \tau}^{j-1}=\sum_{\alpha=0}^{\rho} a_{\alpha,(p-\alpha) p+\tau}^{j, k} .
$$

In view of (5) and (7) it is clear that the equations 
(8) ${ }_{h} F_{p, \tau}^{j-1}=0 \quad(h=0, \cdots, j-1 ; \rho=0,1, \cdots ; \tau=0,1, \cdots, p-1)$

have to be satisfied. It will be necessary to get the left members of (8) in considerable detail. By (7a), (6a), (5b) and (5c) it follows that

$$
\begin{aligned}
{ }_{h} F_{\rho, \tau}^{j-1}= & \sum_{\alpha=0}^{\rho} \sum_{\beta=0}^{(\rho-\alpha) p+r} \sum_{\beta=0}^{j-h-1} \sum_{k=0}^{n} C_{\beta}^{h+\beta}(-1)^{\alpha+\beta} l_{\alpha, \beta} k^{\alpha} \\
& X_{h+\beta \eta_{s}}^{j-1}[k] d_{n-k,(\rho-\alpha) p+r-\rho} \\
= & { }_{h} F_{\rho, \tau: 1}^{j-1}+{ }_{h} F_{\rho, \tau: 2}^{j-1},
\end{aligned}
$$

where

(9a) ${ }_{h} F_{\rho, \tau: 1}^{j-1}=\sum_{\alpha=0}^{\rho} \sum_{\beta=0}^{j-h-1} \sum_{k=0}^{n} C_{\beta}^{h+\beta}(-1)^{\alpha+\beta} l_{\alpha, \beta} \quad{ }_{h+\beta} \eta_{0}^{j-1} k^{\alpha} d_{n-k,(p-\alpha) p+\tau}$,

$$
\begin{aligned}
{ }_{h} F_{\rho, \tau: 2}^{j-1}= & \sum_{\alpha=0}^{\rho} \sum_{\lambda=0}^{\rho-\alpha} \sum_{\substack{w=1 \\
j-1}}^{p} \sum_{\beta=0}^{j-h-1} \sum_{k=0}^{n} C_{\beta}^{h+\beta}(-1)^{\alpha+\beta} l_{\alpha, \beta} k^{\alpha} \\
& \times{ }_{h+\beta} \eta_{\lambda p+w}[k] d_{n-k,(\rho-\alpha-\lambda) p+\tau-w} \quad\left(d_{i, j}=0 \text { for } j<0\right) .
\end{aligned}
$$

For convenience let

$$
\sum_{k=0}^{n} k^{\nu} d_{n-k, i}=\delta_{i}^{\prime} \quad\left(\delta_{i}^{\prime}=0 \text { for } i<0\right) .
$$

On using the relation

$$
\sum_{\lambda=0}^{\rho-\alpha} \sum_{\nu=0}^{\lambda}=\sum_{\nu=0}^{\rho-\alpha} \sum_{\lambda=\nu}^{p-\alpha}
$$

by virtue of $(2 b)$ we have from $(9 b)$

$$
\begin{aligned}
{ }_{h} F_{\rho, \tau: 2}^{j-1}= & \sum_{\beta=0}^{j-h-1} \sum_{w=1}^{p} \sum_{\alpha=0}^{p} \sum_{\nu=0}^{p-\alpha}\left(\sum_{\lambda=\nu}^{p-\alpha} C_{\beta}^{h+\beta} C_{\lambda-\nu}^{-(p p+w) / p}(-1)^{\alpha+\beta} l_{\alpha, \beta}\right. \\
& \left.\times \delta_{(p-\alpha-\lambda) p+\tau-w}^{\lambda-\nu+\alpha}\right)_{h+\beta} \eta_{\nu p+w}^{j-1} .
\end{aligned}
$$

An application of the relationship

$$
\sum_{\alpha=0}^{p} \sum_{\nu=0}^{\rho-\alpha}=\sum_{\nu=0}^{p} \sum_{\alpha=0}^{\rho-\nu}
$$

to (11) will give from (9)

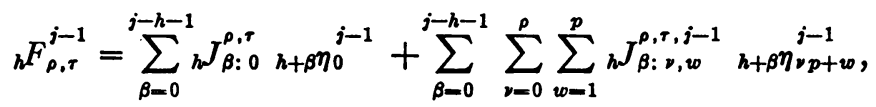


where

$$
\begin{aligned}
{ }_{w} J_{\beta: 0}^{\rho, \tau} & =\sum_{\alpha=\beta}^{\rho} C_{\beta}^{h+\beta}(-1)^{\alpha+\beta} l_{\alpha, \beta} \delta_{(p-\alpha) p+\tau}^{\alpha}, \\
{ }_{h} J_{\beta: \nu, w}^{\rho, \tau, j-1} & =\sum_{\alpha=\beta}^{\rho-\gamma} \sum_{\lambda=\nu}^{\rho-\alpha} C_{\beta}^{h+\beta} C_{\lambda-\nu}^{-(\nu p+w) / p}(-1)^{\alpha+\beta} l_{\alpha, \beta} \delta_{(p-\alpha-\lambda) p+\tau-w}^{\lambda-\gamma+\alpha} .
\end{aligned}
$$

The following definition will be introduced.

Definition. $A$ number $\delta_{i}^{\prime}(c f .(10))$ is of index $\sigma$ provided $i=(\sigma-\nu) p+\zeta$, where $0 \leqq \zeta \leqq p-1$ and $\sigma \geqq \nu$.

In view of the assumed existence of a set of $\phi$ solutions (1), for which (1b) holds, the characteristic equation $(5 ; \S 8)$ will have $\rho=0$ as a root whose multiplicity is precisely $\phi$. As a consequence,

$$
\sum_{k} d_{n-k, 0} k^{i}=\delta_{0}^{i}=0 \quad(i=0,1, \cdots, \phi-1),
$$

but

$$
\sum_{k} d_{n-k, 0} k^{\phi}=\delta_{0}^{\phi} \neq 0 \text {. }
$$

Thus, not all the $\delta_{i}{ }^{\nu}$ of index $\phi$ are zero. It will now be proved that the $\delta_{i}$ ' with indices $0,1, \cdots, \phi-1$ are necessarily all zero.

We have

$$
{ }_{j-1} F_{0,0}^{j-1}={ }_{j-1} J_{0: 0}^{0,0}{ }_{j-1}^{j-1} \eta_{0}^{j-1}=0 \quad(j=1, \cdots, \phi),
$$

where ${ }_{j-1} J_{0: 0}^{0,0}=\delta_{0}^{0}$. As is seen from (13), $\delta_{0}^{0}=0$; but, apart from that, $\delta_{0}^{0}$ would necessarily have to be zero since the numbers (1c) are not all zero. It follows that

$$
{ }_{h} J_{0: 0}^{0,0}=0 \quad(h=j-1, \cdots, 0) .
$$

Suppose

$$
\begin{aligned}
{ }_{h} J_{0,0}^{0, i} & =0 \\
(h=j-1, \cdots, 0 ; i & =0,1, \cdots, \tau-1 ; 1 \leqq \tau \leqq p-1) .
\end{aligned}
$$

In (14) the truth of (14a) has been established for $\tau=1$. On making use of (14a) we obtain

$$
{ }_{j-1} F_{0, \tau}^{j-1}={ }_{j-1} J_{0: 0}^{0, \tau}{ }_{j-1}^{j} \eta_{0}^{j-1}=0 .
$$

Thus; in view of (12a), 


$$
{ }_{h} J_{0: 0}^{0, \pi}=0 \quad(h=j-1, \cdots, 0) .
$$

It follows by induction that (14b) holds for $\tau=0, \cdots, p-1$. Assume now, more generally, that

$$
\begin{gathered}
{ }_{h} J_{\beta: 0}^{i, \tau}=0 \\
(h=j-1, \cdots, 0 ; \beta=0,1, \cdots, i ; \tau=0,1, \cdots, p-1),
\end{gathered}
$$

for $i=0, \cdots, \rho-1(1 \leqq \rho \leqq \phi-1)$. For $\rho=1$ (15) has been proved in (14b). On taking equations $(15 ; h=0)$ in succession for $\beta=i, \beta=i-1, \cdots, 0$, and at each step using the relations previously obtained, we find that

$$
\begin{aligned}
l_{i, i} \delta_{\tau}^{i}=l_{i-1, i-1} \delta_{p+\tau}^{i-1} & =\cdots=l_{0,0} \delta_{i p+\tau}^{0}=0 \\
(\tau & =0,1, \cdots, p ; i=0,1, \cdots, \rho-1) .
\end{aligned}
$$

Accordingly, (15) implies that all the $\delta_{i}^{\nu}$ whose indices are $0,1, \cdots, \rho-1$ are zero.

In the sequel use will be made of the fact that the $\delta_{i}{ }^{7}$ in (12a) are of index $\rho$ and that in (12b) the $\delta_{i}{ }^{\prime}$ are of index $\rho-\nu-1$, for $w>\tau$, and the $\delta_{i}{ }^{\prime}$ are of index $\rho-\nu$, when $w \leqq \tau$. In deriving further consequences of (15) consider

$$
{ }_{j-1} F_{\rho, \tau}^{j-1}=0,{ }_{j-2}^{j} F_{\rho, \tau}^{j-1}=0, \cdots,{ }_{j-\rho-1} F_{\rho, \tau}^{j-1}=0 \quad(\tau=0,1, \cdots, p-1) .
$$

Whenever necessary $j$ will be taken $\geqq \rho-1$. By (15)

$$
{ }_{j-1} F_{\rho, 0}^{j-1}={ }_{j-1} J_{0: 0 \quad j-1}^{\rho, 0} \eta_{0}^{j-1}
$$

so that necessarily

$$
{ }_{h} J_{0: 0}^{p, 0}=0 \quad(h=j-1, \cdots, 0) .
$$

On assuming, more generally,

$$
\begin{gathered}
{ }_{h} J_{\beta: 0}^{p, 0}=0 \\
(h=j-1, \cdots, 0 ; \beta=0,1, \cdots, H-1 ; 1 \leqq H \leqq \rho),
\end{gathered}
$$

it is observed that

$$
\begin{aligned}
{ }_{j-1-H} F_{\rho, 0}^{j-1}= & { }_{j-1-H} J_{H: 0}^{\rho, 0} j_{-1}^{j} \eta_{0}^{j-1}+\sum_{\beta=0}^{H-1}{ }_{j-1-H} J_{\beta: 0}^{\rho, 0} j_{j-1-H+\beta} \eta_{0}^{j-1} \\
& +\sum_{\beta=0}^{B} \sum_{\nu=0}^{\rho-1} \sum_{w=1}^{p}{ }_{j-1-H} J_{\beta: \nu, w}^{\beta, 0, j-1} .
\end{aligned}
$$

In consequence of $(16 \mathrm{~b})$ and since the $\delta_{i}^{\nu}$ in the ${ }_{h} J_{\beta: \nu, w}^{p, 0, j-1}$ have indices $\leqq \rho-1$, 
it follows, in view of the preceding italicized statement, that

$$
{ }_{j-1-H} F_{\rho, 0}^{j-1}={ }_{j-1-H} J_{y: 0}^{\rho, 0} \quad j_{j-1}^{j-1} \eta_{0}^{j-1}=0 .
$$

(16c) implies that

$$
{ }_{h} J_{H: 0}^{p, 0}=0 \quad(h=j-1, \cdots, 0) .
$$

As (16d) follows from (16b),

$$
{ }_{h} J_{\beta: 0}^{\rho, 0}=0 \quad(h=j-1, \cdots, 0 ; \beta=0,1, \cdots, \rho) .
$$

Assume that, for $\tau=0,1, \cdots, \sigma-1(1 \leqq \sigma \leqq p-1)$,

$$
{ }_{n} J_{\beta: 0}^{\rho, \tau}=0 \quad(h=j-1, \cdots, 0 ; \beta=0,1, \cdots, \rho) ;
$$

in (17) these relations have been proved for $\sigma=1$. In view of (12a), equations (18), when examined in succession for $\beta=\rho, \rho-1, \cdots, 0$ (any $h$ ), will be seen to imply

$$
\delta_{\tau}^{0}=\delta_{p+\tau}^{\rho-1}=\cdots=\delta_{\rho p+\tau}^{0}=0 \quad(\tau=0,1, \cdots, \sigma-1) .
$$

A further consequence would be the relations

$$
{ }_{j-1} J_{0: 0, w}^{p, \sigma, j-1}=0 \quad(1 \leqq w \leqq \sigma) .
$$

On making use of (15a) and (18b) it is observed that

$$
{ }_{j-1} F_{p, 0}^{j-1}={ }_{j-1}^{0} J_{0: 0}^{0,0} j_{-1}^{j} \eta_{0}^{j-1}=0
$$

so that

$$
{ }_{h} J_{0: 0}^{\rho, 0}=0 \quad(h=j-1, \cdots, 0) .
$$

Assume now a set of relations, more general than (19),

$$
{ }_{h} J_{\beta: 0}^{\rho, \sigma}=0 \quad(h=j-1, \cdots, 0),
$$

where $\beta=0,1, \cdots, H-1(1 \leqq H \leqq p)$. On account of (18a) and (19a) and by virtue of certain previously established facts, the relations

$$
{ }_{j-1-H} F_{p, \Theta}^{j-1}={ }_{j-1-H} J_{H: 0}^{\rho . e} ;-1 \eta_{0}^{f-1}=0
$$

would then follow; that is,

$$
{ }_{W} J_{H: 0}^{\rho, \bullet}=0 \quad(h=j-1, \cdots, 0) .
$$

Hence, by induction, 


$$
{ }_{h} J_{\beta: 0}^{\rho, \sigma}=0 \quad(h=j-1, \cdots, 0)
$$

for $\beta=0,1, \cdots, \rho$. On noting that (20) is a consequence of $(18)$, it is observed that an induction in a more extended sense has been completed. Thus, for $\tau=0,1, \cdots, p$,

$$
{ }_{h} J_{\beta: 0}^{\rho, \tau}=0 \quad(h=j-1, \cdots, 0 ; \beta=0,1, \cdots, \rho) .
$$

Therefore, it is observed that if (15) holds for $i=0, \cdots, \rho-1$, as originally assumed, necessarily (15) will also hold for $i=\rho$. Whence it follows that

$$
\begin{gathered}
{ }_{h} J_{\beta: 0}^{i, \tau}=0 \\
(h=j-1, \cdots, 0 ; \beta=0,1, \cdots, i ; \tau=0,1, \cdots, p-1)
\end{gathered}
$$

for $i=0,1, \cdots, \phi-1$. Just as (15a) has been established on the basis of (15), we now conclude that all the $\delta_{i}{ }^{\prime}$, whose indices are $0,1, \cdots, \phi-1$, are zero. Thus, the italicized statement following (13a) has been proved.

In view of the fact just established, we obtain from (12a) and (12b)

$$
\begin{array}{rlr}
{ }_{h} J_{\beta: 0}^{\rho, \tau} & =0 \\
{ }_{h} J_{\beta: \nu, w}^{\rho, \tau, j-1} & =0\left\{\begin{array}{lr}
\rho \leqq \phi-1 & (\rho \leqq \phi-1), \\
\rho \leqq \phi & (w \leqq \tau),
\end{array}\right.
\end{array}
$$

Thus, relations (8) are all satisfied for $\rho=0,1, \cdots, \phi-1$, without yielding any information about the coefficients ${ }_{k} \eta_{s}^{j-1}$.

In view of the statement preceding (16),

$$
{ }_{h} J_{\beta: \nu, w}^{\rho, \tau, j-1}=0 \begin{cases}\nu \geqq \rho-\phi+1 & (w \leqq \tau), \\ \nu \geqq \rho-\phi & (w>\tau) .\end{cases}
$$

Accordingly equations (8), which need to be considered only for $\rho \geqq \phi$. can be written in the form

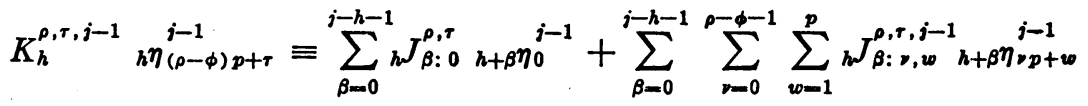

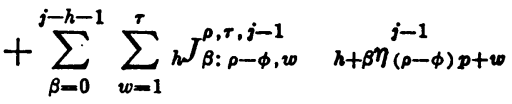

$$
\begin{aligned}
& (\rho=\phi, \phi+1, \cdots ; h=j-1, \cdots, 0 ; \tau=0,1, \cdots, p-1) \text {, }
\end{aligned}
$$

where

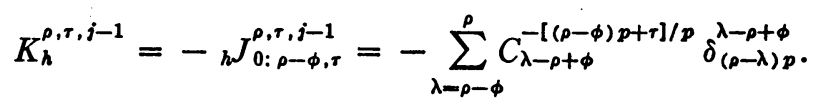


In the second member of (25) the term containing ${ }_{h} \eta_{(\rho-\phi) p+r}^{j-1}$ is omitted. From (25), with $\rho=\phi, h=j-1, \tau=0$, it follows that necessarily

$$
\delta_{\phi p}^{0}=0 \text {. }
$$

The essential fact is noted that in view of (13a) not all the terms in the expression for $K_{h}^{p, r, j-1}$ may be zero. It is clear, then, that the coefficient of $\eta^{j-1}$ in the left member of (25) may vanish only for a finite number of values of the involved subscripts and superscripts. Equations (25) may be solved according to the scheme

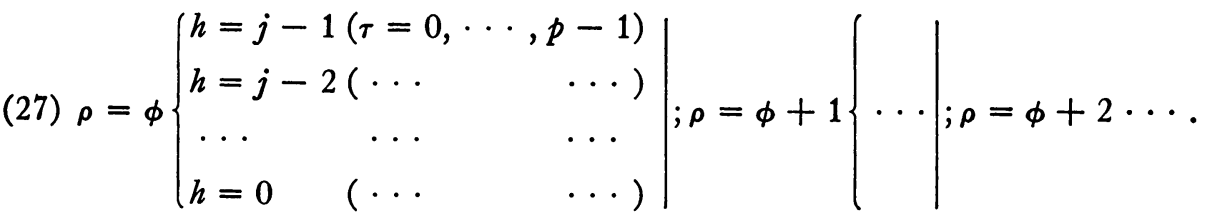

In connection with the possible vanishing of some of the numbers defined by (25a), a statement can be made precisely analogous to that preceding Lemma 1 ( $\$ 3)$.

LEMma 6. Consider a root, of multiplicity $\phi$, of the characteristic equation $(5, \S 8)$ associated with the difference equation $\left(\mathrm{B}_{1}\right)$. In order that, corresponding to this root, there should exist a linearly independent set of $\phi$ formal solutions of type (1), (1a), (1b) the following conditions are necessary and sufficient.

$$
\delta_{0}^{\phi} \neq 0, \quad \delta_{\phi p}^{0}=0 .
$$

(ii) All the $\delta_{i}{ }^{\nu}$, whose indices (cf. Definition) are $0,1, \cdots, \phi-1$, are zero.

(iii) If any of the $K_{h^{\rho, \tau}, j-1}(\rho \geqq \phi)$, defined by (25a), are zero (cf. italics preceding (27)) then the $\delta_{i}{ }^{\prime}$ satisfy conditions implied by the vanishing of the corresponding second members of (25).

10. The mixed system of difference equations. The formal solutions $(1 ; \S 9),(1 \mathrm{a} ; \S 9),(1 \mathrm{~b} ; \S 9)$ may be written in the form

$$
\begin{aligned}
& y_{j}(x)\left(=s_{j}(x)\right)=\sum_{h=0}^{j-1} \log ^{h} x\left[{ }_{h} \eta_{0}^{j-1}+\sum_{w=1}^{p} x^{(p-w) / p}{ }_{h \eta_{w}^{j-1}}^{j-x}(x)\right] \\
& (j=1, \cdots, \dot{\phi}),
\end{aligned}
$$

where

$$
{ }_{h \eta_{w}}^{j-1}(x)=\sum_{\lambda=0}^{\infty}{ }_{h \eta_{\lambda p+w}^{j-1}}^{j-\lambda-1}
$$


The coefficients in the series (1a) are specified as in $\$ 9$. Let $j$ for the present have a fixed value. Consider (1) as a transformation to be applied on the difference equation $\left(B_{1}\right)$. The coefficients of $\left(B_{1}\right)$ may be written in the form

$$
\begin{aligned}
d_{n-k}(x) & =d_{n-k, 0}+\sum_{\nu=1}^{p} x^{(p-p) / p} d_{n-k, \nu}(x), \\
d_{n-k, \nu}(x) & =\sum_{\lambda=0}^{\infty} d_{n-k, \lambda p+\nu} x^{-\lambda-1} .
\end{aligned}
$$

From (1), in view of $(3 b ; \S 9)$, it follows that

$$
y_{j}(x+k)=Y_{k: 1}^{j}+Y_{k: 2}^{j},
$$

where

$$
\begin{aligned}
Y_{k: 1}^{j} & =\sum_{h=0}^{j-1} \log ^{h} x \sum_{\beta=0}^{j-1-h} \sum_{\alpha=0}^{\infty} C_{\beta}^{h+\beta}(-1)^{\alpha+\beta} l_{\alpha, \beta} k_{h+\beta}^{\alpha} \eta_{0}^{j-1} x^{-\alpha}, \\
Y_{k: 2}^{j} & =\sum_{h=0}^{j-1} \sum_{w=1}^{p} \log ^{h} x x^{(p-w) / p} \sum_{\beta=0}^{j-1-h}{ }_{h=0}^{j-1} A_{k: \beta, w}{ }_{h+\beta} \eta_{w}^{j-1}(x) .
\end{aligned}
$$

In $(3 b)$

$$
{ }_{h} A_{k: \beta, w}^{j-1}=\sum_{\alpha=0}^{\infty} \sum_{\delta=0}^{\infty} C_{\beta}^{h+\beta} C_{\delta}^{(p-w) / p}(-1)^{\alpha+\beta} l_{\alpha, \beta} k^{\alpha+\delta} x^{-\alpha-\beta} .
$$

On making use of $(7 ; \S 4)$ it is observed that, by virtue of $(2),(2 b),(3)$, (3a), (3b), (3c),

$$
L\left(y_{j}(x)\right) \equiv L_{1}^{j}+L_{2}^{j}
$$

Here

$$
L_{1}^{j}=\sum_{h=0}^{j-1} \sum_{w=1}^{p} x^{(p-w) / p} \log ^{h} x \sum_{\beta=0}^{j-1-h} \sum_{k=0}^{n}{ }_{h, w} L_{\beta, k}^{j-1}
$$

where

$$
\begin{aligned}
& { }_{h, w} L_{\beta, k}^{j-1}=\sum_{\xi=1}^{w-1} \sum_{\alpha, \delta, \lambda=0}^{\infty} C_{\beta}^{h+\beta} C_{\delta}^{(p-\zeta) / p}(-1)^{\alpha+\beta} l_{\alpha, \beta} k^{\alpha+\delta} d_{n-k, \lambda p+w-\zeta} \\
& \times x^{-\alpha-\delta-\lambda}{ }_{h+\beta \eta}^{j-1}(x+k)+\sum_{\xi=\omega}^{p} \sum_{\alpha, \delta, \lambda=0}^{\infty} C_{\beta}^{h+\beta} C_{\delta}^{(p-\zeta) / p}(-1)^{\alpha+\beta} l_{\alpha, \beta} k^{\alpha+\infty} \\
& \times d_{n-k, \lambda p+p+w-\zeta} x^{-\alpha-\delta-\lambda-1}{ }_{n+\beta \eta \zeta}^{j-1}(x+k)+\sum_{\alpha, \delta=0}^{\infty} C_{\beta}^{h+\beta} C_{\delta}^{(p-w) / p} \\
& \times(-1)^{\alpha+\beta} l_{\alpha, \beta} k^{\alpha+\delta} d_{n-k, 0} x^{-\alpha-\beta}{ }_{h+\beta}^{j-1}(x+k) \text {; }
\end{aligned}
$$


and

$$
\begin{aligned}
L_{2}^{j}= & \sum_{h=0}^{j-1} \sum_{w=1}^{p} \log ^{h} x x^{(p-w) / p} \sum_{\alpha, \lambda=0}^{\infty} \sum_{\beta=0}^{j-1-h} C_{\beta}^{h+\beta}{ }_{h+\beta \eta_{0}^{j-1}}(-1)^{\alpha+\beta} l_{\alpha, \beta} \\
& \times \delta_{\lambda p+w}^{\alpha} x^{-\alpha-\lambda-1}+\sum_{h=0}^{j-1} \log ^{h} x \sum_{\alpha=0}^{\infty} \sum_{\beta=0}^{j-1-h} C_{\beta}^{h+\beta}{ }_{h+\beta \eta_{0}^{j-1}}^{j-1}(-1)^{\alpha+\beta} l_{\alpha, \beta} \delta_{0}^{\alpha} x^{-\alpha} .
\end{aligned}
$$

On writing

$$
L\left(y_{j}(x)\right) \equiv \sum_{h=0}^{j-1} \sum_{w=1}^{p} \log ^{h} x x^{(p-w) / p}{ }_{j} W_{h, w},
$$

it is observed that, in view of the involved formal facts, the relations ${ }_{j} W_{h, w}=0(h=0, \cdots, j-1 ; w=1, \cdots, p)$ have to be satisfied; these equations may be written in the form

$$
\begin{aligned}
& T_{h, w}^{j-1} \equiv \sum_{\beta=0}^{j-1-h} \sum_{k=0}^{n} \sum_{\zeta=1}^{p}{ }_{\beta} a_{\zeta, k}^{h, w}(x){ }_{h+\beta \eta \zeta}^{j-1}(x+k)=g^{h, w, j}(x) \\
&(h=0, \cdots, j-1 ; w=1, \cdots, p) .
\end{aligned}
$$

Here, by virtue of (4), (4a), (4b) and (4c), the coefficients are series of the form

$$
\begin{aligned}
& { }_{\beta} a_{\zeta, k}^{h, w}(x)=\sum_{a=0}^{\infty}{ }_{\beta} a_{\xi, k: s}^{h, w} x^{-\boldsymbol{s}}, \\
& g^{h, w, j}(x)=-\sum_{\varepsilon=1}^{\infty} g_{\ell}^{h, w, i} x^{-\ell},
\end{aligned}
$$

where

(6) $\quad 0 a_{5, k: 0}^{h, w}=d_{n-k, w-5}$

$$
(\zeta \leqq w),
$$

(6a) ${ }_{\beta} a_{5, k: 0}^{h, w}=0$

$(\zeta>w)$,

${ }_{\beta}^{h} a_{\xi, k: 0}^{h, w}=0$

$(\beta \geqq 1 ; \zeta \leqq w)$,

and, for $s \geqq 1$,

(7) ${ }_{\beta} a_{\zeta, k: s}^{h, w}=\sum_{\lambda=0}^{\dot{s}} \sum_{\alpha=0}^{s-\lambda} C_{\beta}^{h+\beta} C_{s-\alpha-\lambda}^{(p-\zeta) / p}(-1)^{\alpha+\beta} l_{\alpha, \beta} d_{n-k, \lambda p+w-\zeta} k^{s-\lambda}$

(7a) ${ }_{\beta} a_{w, k: \diamond}^{h, w}=\sum_{\alpha=0}^{s} C_{\beta}^{h+\beta} C_{o-\alpha}^{(p-w) / p}(-1)^{\alpha+\beta} l_{\alpha, \beta} k^{*} d_{n-k, 0}$

$$
+\sum_{\lambda=0}^{\circ-1} \sum_{\alpha=0}^{\rho-1-\lambda} C_{\beta}^{h+\beta} C_{s-1-\alpha-\lambda}^{(p-w) / p}(-1)^{\alpha+\beta} l_{\alpha, \beta} k^{s-1-\lambda} d_{n-k,(\lambda+1) p},
$$

(7b) ${ }_{\beta} a_{\zeta, k: s}^{h, w}=\sum_{\lambda=0}^{s-1} \sum_{\alpha=0}^{\circ-\lambda-1} C_{\beta}^{h+\beta} C_{s-\alpha-\lambda-1}^{(p-\zeta) / p}(-1)^{\alpha+\beta} l_{\alpha, \beta} k^{s-\lambda-1} d_{n-k, \lambda p+p-\zeta+w} \quad(\zeta>w)$. 
The coefficients in (5a) may be computed with the aid of (4c). We have, on using the notation $(10 ; \S 9)$,

$$
\begin{aligned}
g^{h, w, i}(x)= & \sum_{\alpha, \lambda=0}^{\infty} \sum_{\beta=0}^{j-1-h} C_{\beta}^{h+\beta}(-1)^{\alpha+\beta+1} l_{\alpha, \beta} \quad h+\beta \eta_{0}^{j-1} \delta_{\lambda p+w}^{\alpha} x^{-\alpha-\lambda-1} \\
& +\xi(w) \sum_{\beta=0}^{j-1-h} \sum_{\alpha=0}^{\infty} C_{\beta}^{h+\beta}(-1)^{\alpha+\beta+1} l_{\alpha, \beta} \quad h+\beta \eta_{0}^{j-1} \delta_{0}^{\alpha} x^{-\alpha} ;
\end{aligned}
$$

here $\xi(p)=1$ and $\xi(w)=0$ when $w \neq p$. Thus,

$$
\begin{aligned}
-g_{s}^{h, w, j}= & \sum_{\beta=0}^{j-1-h} \sum_{\alpha=0}^{s-1} C_{\beta}^{h+\beta}(-1)^{\alpha+\beta+1} l_{\alpha, \beta} h_{h+\beta} \eta_{0}^{j-1} \delta_{(\delta-1-\alpha) p+w}^{\alpha} \\
& +\xi(w) \sum_{\beta=0}^{j-1-h} C_{\beta}^{h+\beta}(-1)^{s+\beta+1} l_{s, \beta} \quad h_{+\beta} \eta_{0}^{j-1} \delta_{0}^{\varepsilon} .
\end{aligned}
$$

The series (5), (5a) all converge in the vicinity of infinity. Furthermore, it is seen that the "mixed" difference system $\left(B_{2}\right)$ is formally satisfied by the possibly divergent series ${ }_{h+\beta} \eta_{5}^{j-1}(x)$ (cf. (1a)).

LEмма 7. Write the $\phi$ formal solutions $((1 ; \S 9),(1 \mathrm{a} ; \S 9),(1 \mathrm{~b} ; \S 9))$ [corresponding to a root of multiplicity $\phi$ of the characteristic equation $(5 ;$;8) associated with the difference equation $\left.\left(\mathrm{B}_{1}\right)\right]$ in the form (1), (1a). The formal series (1a) (with $j$ fixed) will satisfy a "mixed" difference system $\left(\mathrm{B}_{2}\right)$ whose coefficients are given by series (5), (5a), (6), (6a), (7), (7a), (7b), (8a), all convergent in a neighborhood of $x=\infty$.

11. The corresponding system of integral equations. It is clear that whenever the characteristic equation $E_{1}(t)=0(5 ; \S 8)$ possesses a root $t=\rho$, the numbers $\rho \pm 2 \pi i \nu(\nu=1,2, \cdots)$ will also be roots. In particular, this equation has roots, on the axis of imaginaries, associated with the root $t=0$. Regions $P$ and $S$ will be defined as follows.

Let $P$ denote the half $t$-plane $R t \geqq 0$, excluding small sectors each with vertex at $t=0$ and containing the non-zero roots of $E_{1}(t)=0$ in their interiors.

Let $S$ denote a strip

$$
\epsilon \leqq t_{1} \leqq a ; t_{2} \geqq 0 \quad\left(0<\epsilon<a ; t=t_{1}+i t_{2}\right),
$$

not containing any of the roots of $E_{1}(t)=0$.

In the sequel, whenever the integrals

$$
\int_{0}^{\infty} \quad \int_{0}^{t}
$$

are said to be extended in $P$, it will be understood that the path of integration 
is along a ray $\angle t=\bar{t}$, extending from the origin and situated in $P$. On the other hand, when these integrals are said to be extended in $S$, the supposition will be that the path is from $t=0$ along the positive axis of reals up to some point $t=t_{1}\left(\epsilon \leqq t_{1} \leqq a\right)$; from the latter point the path will be assumed to extend, in $S$, along the ray $R t=t_{1}$.

The variable $x$ will be so restricted that

$$
\lim _{t \rightarrow \infty}\left|e^{t x} t^{\alpha}\right|=0 \quad(\text { every } \alpha>0)
$$

along the ray $\angle t=\bar{t}$, under consideration, of $P$; or along a line, $R t=t_{1}$, extending in $S$. We then have formally

$$
a(x)=\sum_{s=1}^{\infty} a_{s} x^{-s}=\int_{0}^{\infty} \tilde{a}(t) e^{t x} d t \quad \text { (integration in } P \text { or } S \text { ), }
$$

where

$$
\tilde{a}(t)=\sum_{\nu=1}^{\infty} \tilde{a}_{\nu} t^{\nu-1} ; \quad \tilde{a}_{\nu}=\frac{(-1)^{\nu} a_{\nu}}{(\nu-1) !} .
$$

Unless stated explicitly integrations below are in $P$ or $S$.

The series $(1 \mathrm{a} ; \S 10)$ are formally representable as follows:

$$
{ }_{h \eta_{w}}^{j-1}(x)=\int_{0}^{\infty}{ }_{h} \tilde{\eta}_{w}^{j-1}(t) e^{t x} d t
$$

where

$$
{ }_{h} \tilde{\eta}_{w}^{j-1}(t)=\sum_{\nu=0}^{\infty}{ }_{h} \tilde{\eta}_{w: \nu}^{j-1} t ; \quad{ }_{h} \tilde{\eta}_{w: \nu}^{j-1}=\frac{(-1)^{\nu+1}}{\nu !}{ }_{h}{ }_{\eta}^{j-1} \eta_{p+w}^{j-1} .
$$

Consider now (3) as a transformation on the dependent variables to be applied to the difference system $\left(B_{2}\right)$ (Lemma 7 ). It is not practicable to establish convergence of the series $(3 \mathrm{a})$ by making use of the relations $(25 ; \S 9)$. Instead use will be made of a system of integral equations.

We have formally

$$
{ }_{h+\beta}^{j-\beta} \eta_{5}^{j-1}(x+k)=\int_{0}^{\infty}\left({ }_{h+\beta} \tilde{\eta}_{5}^{j-1}(t) e^{k t}\right) e^{t x} d t
$$

and, for $\lambda=1,2, \cdots$,

$$
x^{-\lambda}{ }_{h+\beta}^{j-1} \eta_{\zeta}^{j-1}(x+k)=-\int_{0}^{\infty}\left[\int_{0}^{t} \frac{(\tau-t)^{\lambda-1}}{(\lambda-1) !} e^{k+\beta}{ }_{h+\beta}^{j-1} \eta_{\zeta}^{j-1}(\tau) d \tau\right] e^{t x} d t
$$


provided

(5)

$$
\left[e^{t x} \int_{0}^{t} e^{k t}{ }_{h} \tilde{\eta}_{w}^{j-1}(t) d t^{(H)}\right]_{0}=0
$$

$(k=0,1, \cdots, n ; h=0, \cdots, j-1 ; w=1, \cdots, p ; H=1,2, \cdots)$. $^{*}$

By $(2)$ and $(2 a)$ the second members $(5 a ; \S 10)$ of $\left(B_{2}\right)$ are expressible in the form

$$
\begin{aligned}
& g^{h, w, j}(t)=\int_{0}^{\infty} \tilde{g}^{h, w, j}(t) e^{t x} d t \\
& \tilde{g}^{h, w, j}(t)=\sum_{\nu=1}^{\infty} \tilde{g}_{\nu}^{h, w, j} t^{-1}, \quad \tilde{g}_{\nu}^{h, w, j}=\frac{(-1)^{\nu+1}}{(\nu-1) !} g_{\nu}^{h, w, j} ;
\end{aligned}
$$

moreover, by (4) and (4a),

$$
\begin{aligned}
{ }_{\beta} a_{\zeta, k}^{h, w}(x)_{h+\beta} \eta_{\zeta}^{j-1}(x & +k)=\int_{0}^{\infty} e^{t x}\left[{ }_{\beta} a_{\zeta, k: 0}^{h, w} e^{k t}{ }_{h+\beta} \tilde{\eta}_{\zeta}^{j-1}(t)\right. \\
& \left.-\int_{0}^{t}\left(\sum_{\lambda=1}^{\infty}{ }_{\beta} a_{\zeta, k: \lambda}^{h, \alpha} \frac{(\tau-t)^{\lambda-1}}{(\lambda-1) !} e^{k \tau}\right)_{h+\beta} \tilde{\eta}_{\zeta}^{j-1}(\tau)\right] d t .
\end{aligned}
$$

On substituting (6) and (7) in $\left(B_{2}\right)$ it is found that the system $\left(B_{2}\right)$ is formally satisfied if

$$
\begin{aligned}
& \sum_{\beta=0}^{j-h-1} \sum_{\zeta=1}^{p}{ }_{\beta} b_{\zeta}^{h, w}(t)_{h+\beta} \tilde{\eta}_{\zeta}^{j-1}(t) \\
& =\sum_{\beta=0}^{j-1-h} \sum_{\zeta=1}^{p} \int_{0}^{t}{ }_{\beta} C_{\zeta}^{h, w}(t, \tau){ }_{h+\beta} \tilde{\eta}_{\zeta}^{j-1}(\tau) d \tau+\tilde{g}^{h, w, j}(t) \\
& \quad(h=0, \cdots, j-1 ; w=1, \cdots, p ; j=1, \cdots, \phi) .
\end{aligned}
$$

In the system (8)

$$
\begin{aligned}
{ }_{\beta} b_{\zeta}^{h, w}(t) & =\sum_{k=0}^{n}{ }_{\beta} a_{\zeta, k: 0}^{h, w} e^{t k}, \\
{ }_{\beta} C_{\zeta}^{h, w}(t, \tau) & =\sum_{\lambda=1}^{\infty} \sum_{k=0}^{n}{ }_{\beta} a_{\zeta, k: \lambda}^{h, w} \frac{(\tau-t)^{\lambda-1}}{(\lambda-1) !} e^{k \tau},
\end{aligned}
$$

where the ${ }_{\beta} a_{\zeta, k: \lambda}^{h, w}$ are given by the formulas (8), (8a), (9), (9a), (9b) of $\$ 10$. In view of the convergence of the series $(2 b ; \S 10)$ and by virtue of $(8 a ; \S 10)$ and of (6a) it follows that

* The formal steps will be finally justified. 


$$
\left|{ }_{\beta} a_{\xi, k: \lambda}^{h, w}\right|, \quad\left|g_{\lambda}^{h, w, i}\right|<R_{\rho}^{\lambda} \quad(\lambda=0,1, \cdots) .
$$

Thus the coefficients of the system (8) are entire in the involved variables. The system ( 8 ) is formally satisfied by the series (3a).

As a consequence of $(6 ; \S 10)$ and $(6 a ; \S 10)$ we have

$$
0 b_{\zeta}^{h, w}(t)=\sum_{k=0}^{n} d_{n-k, w-\zeta} e^{k t}=b^{w-\zeta}(t) \quad(\zeta=1, \cdots, w),
$$

(10a) ${ }_{\beta} b_{\xi}^{h, w}(t)=0$

$(\zeta>w)$,

${ }_{\beta} b_{\zeta}^{h, w}(t)=0$

$(\beta \geqq 1 ; \zeta \leqq w)$.

Accordingly the first member of (8) is replaced by

$$
\sum_{\zeta=1}^{\infty} b^{20-\zeta}(t) h_{h}^{j-1}(t)
$$

Since, by $(4 \mathrm{a} ; \S 8)$, not all the $d_{n-k, 0}$ are zero it is noted that $b^{0}(t) \not \equiv 0$.

It will be necessary to examine ( $8 b)$ in greater detail. We have

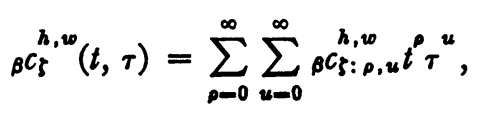

where

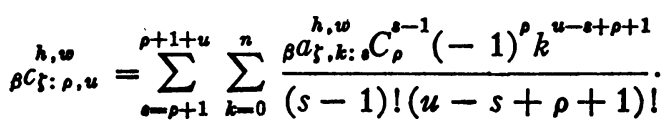

Hence by $(7),(7 a),(7 b)$ of $\$ 10$, on using the notation $(13 ; \S 9)$, it follows that

$$
\begin{aligned}
{ }_{\beta} C_{w: \rho, u}^{h, w}= & \sum_{-\rho+1}^{\rho+1+u} \sum_{\alpha=0}^{\infty} \frac{C_{\beta}^{h+\beta} C_{s-\alpha}^{(p-w) / p} C_{\rho}^{\alpha-1} l_{\alpha, \beta}(-1)^{\rho+\alpha+\beta} \delta_{0}^{u+\rho+1}}{(s-1) !(u-s+\rho+1) !} \\
& +\sum_{-\rho+\rho+1}^{\rho+1+u} \sum_{\lambda=0}^{\rho-1} \sum_{\alpha=0}^{\rho-1-\lambda} \frac{C_{\beta}^{n+\beta} C_{s-1-\alpha-\lambda}^{(p-w) / p} C_{\rho}^{\rho-1} l_{\alpha, \beta}(-1)^{\rho+\alpha+\beta} \delta_{(\lambda+1) p}^{u+\rho-\lambda}}{(s-1) !(u-s+\rho+1) !}
\end{aligned}
$$

and, for $1 \leqq \zeta \leqq w-1$,

(13a) $\underset{\beta C_{\xi: \rho, u}^{h, w}}{h, \rho+1+u}=\sum_{s-\rho+1}^{s} \sum_{\lambda=0}^{s} \sum_{\alpha=0}^{\rho-\lambda} \frac{C_{\beta}^{\mu+\beta} C_{o-\alpha-\lambda}^{(p-\zeta) / p} C_{\rho}^{s-1} l_{\alpha, \beta}(-1)^{\alpha+\beta+\rho}}{(s-1) !(u-s+\rho+1) !} \delta_{\lambda p+w-\zeta}^{u+\rho+1-\lambda}$, while, for $\zeta>w$,

(13b) ${ }_{\beta C_{\xi: \rho, u}}^{h, w}=\sum_{-\rho+1}^{\rho+1+u} \sum_{\lambda=0}^{\rho-1} \sum_{\alpha=0}^{\rho-\lambda-1} \frac{C_{\beta}^{h+\beta} C_{\delta-\alpha-\lambda-1}^{(p-\zeta) / p} C_{\rho}^{\alpha-1} l_{\alpha, \beta}(-1)^{\alpha+\beta+\rho} \delta_{\lambda p+p-\beta+\nu}^{u+\rho-\lambda}}{(s-1) !(u-s+\rho+1) !}$. 
Now the indices (cf. Definition of $\S 9$ ) of the $\delta_{i}$ displayed in (13), (13a) and (13b) are $u+\rho+1, u+\rho+1$ and $u+\rho$, respectively. This, by virtue of the fact that condition (ii) of Lemma $6(\$ 9)$ is satisfied, implies that

$$
\begin{aligned}
& { }_{\beta}^{h C_{\zeta: \rho, u}^{h, w}}=0 \quad(\rho+u \leqq \phi-2 ; \zeta \leqq w), \\
& { }_{\beta}^{h C_{\zeta: \rho, u}^{h, w}}=0 \quad(\rho+u \leqq \phi-1 ; \zeta>w) .
\end{aligned}
$$

As to the properties at infinity of the coefficients in the second members of (8), it is noted that by (6a) and (9)

$$
\left|\tilde{g}^{h, w, j}(t)\right|<R^{\prime \prime} e^{\rho|t|}
$$

moreover, by ( $8 \mathrm{~b})$ and (9),

$$
\left|{ }_{\beta} C_{\xi}^{h, w}(t, \tau)\right|<R^{\prime \prime}\left|e^{n \tau}\right| e^{\rho|t-\tau|}
$$

provided $R \tau \geqq 0$ and $R^{\prime \prime}$ is sufficiently great.

It is observed that the functions (10) are representable by the series

$$
b^{w-5}(t)=\sum_{i=0}^{\infty} \delta_{w-5}^{i} \frac{t^{2}}{i !}
$$

where, by Lemma $6, \delta_{w \rightarrow \zeta}^{i}=0(\zeta=1, \cdots, w ; i=0, \cdots, \phi-1)$. Thus

$$
b^{w-\zeta}(t)=t^{\phi} d^{w-\zeta}(t) \quad(\zeta=1, \cdots, w),
$$

the $d^{w-s}(t)$ being entire in $t$. Furthermore, since $\delta_{0}^{\phi} \neq 0$, the function

$$
\frac{t^{\phi}}{b^{0}(t)}=\frac{t^{\phi}}{E_{1}(t)}=d(t) \quad[\text { cf. }(5 ; \S 8) ; d(0) \neq 0]
$$

is meromorphic and, at $t=0$, it is analytic. $\dagger$ The poles of $d(t)$ are given by the totality of the non-zero roots of the characteristic equation $E_{1}(t)=0$.

On noting that by $(4 \mathrm{a} ; \S 8) d_{m, 0} \neq 0(0 \leqq m)$ it is concluded that, for $t$ in $P$ and for $t$ in $S$,

$$
|d(t)|<d\left|e^{-(n-m) t} t^{\phi}\right| .
$$

On the other hand, in view of (10) and (17),

$$
\left|d^{w-\gamma}(t)\right|<d\left|e^{n t} t-\phi\right| \quad(R t \geqq 0) .
$$

Substituting (10) and (10a) in the left members of (8), we bring the system (8) to the form

$$
\begin{array}{r}
t_{h}^{\phi} \tilde{\eta}_{w}^{j-1}(t)=\sum_{\beta=0}^{j-h-1} \sum_{\zeta=1}^{p} \int_{0}^{t}{ }_{\beta}^{* h, w} C_{\zeta}^{h, w}(t, \tau){ }_{h+\beta} \tilde{\eta}_{\zeta}^{j-1}(\tau) d \tau+{ }_{g}^{* h, w, j}(t) \\
(h=0, \cdots, j-1 ; w=1, \cdots, p),
\end{array}
$$

$\dagger$ With the number $a$, used in the definition of $s$, sufficiently small $d(t)$ is analytic for $|t| \leqq a$. 
where

$$
\begin{gathered}
{ }_{\beta C_{\zeta}}^{*, w}(t, \tau)=d(t){ }_{\beta}^{h C_{\zeta}, w}(t, \tau)-d(t) d^{w-\zeta}(t) \sum_{\sigma=1}^{w-1}{ }_{\beta}^{*} C_{\zeta}^{h, \sigma}(t, \tau), \\
{ }^{*} g^{h, w, j}(t)=d(t) \tilde{g}^{h, w, j}(t)-d(t) d^{w-\zeta}(t) \sum_{\sigma=1}^{w-1}{ }^{k} g^{h, \sigma, j}(t) .
\end{gathered}
$$

The steps to be used in this connection are precisely those employed in establishing equivalence of the system $\left(\mathrm{A}_{3} ; \S 5\right)$ to $(7 ; \S 5)$.

In view of the established properties of the coefficients of the system (8) it follows, by successive applications of the relations (19), (19a), that

$$
\begin{aligned}
& { }_{\beta}^{*} C_{\zeta}^{h, w}(t, \tau)=\sum_{s=0}^{\infty} \sum_{q=0}^{\infty}{ }_{\beta}^{* h, w: w} C_{\zeta, q}^{h, \tau^{q}}, \\
& \stackrel{*}{g}^{* h, w, j}(t)=\sum_{s=0}^{\infty} g_{: s}^{* h, w, j} t^{s}
\end{aligned}
$$

the involved series being convergent for $|t|<\rho^{\prime}$. $\dagger$ Moreover, the coefficients of $\left(B_{3}\right)$ are meromorphic functions (in $t$ ), whose $t$-poles are at the non-zero roots of $E_{1}(t)=0$. The functions (20) are entire in $\tau$.

As a consequence of (14), (14a) and since $d(t)$ and the $d^{w-r}(t)$ are analytic at $t=0$, it follows from (19) and (19a) that

$$
{ }_{\beta}^{* h C_{\zeta: \rho, u}^{h, w}}=0 \begin{cases}\rho+u \leqq \phi-2 & (\zeta \leqq w), \\ \rho+u \leqq \phi-1 & (\zeta>w) .\end{cases}
$$

It will be demonstrated that, for $t$ in $P$ and $S$, and for $R_{0}$ sufficiently great,

$$
\begin{aligned}
& \left|{ }_{\beta}^{*} C_{\xi}^{h, w}(t, \tau)\right|<R_{0}|t|^{\phi}\left|e^{w m t}\right|\left|e^{-n(t-\tau)}\right| e^{p|t-\tau|}, \\
& \left|{ }^{*} g^{h, w, j}(t)\right|<R_{0}|t| \phi\left|e^{w m t}\right|\left|e^{-n t}\right| e^{\rho|t|}
\end{aligned}
$$

$(\beta=0,1, \cdots, j-h-1 ; \zeta=1, \cdots, p ; h=0, \cdots, j-1 ; w=1, \cdots, p ; R \tau \geqq 0)$.

In fact, by (18) and (16),

$$
\left|{ }_{\beta}^{* h, 1}(t, \tau)\right|=\left.|d(t)|{ }_{\beta C_{\zeta}}^{h, 1}(t, \tau)\left|<d R^{\prime \prime}\right| t\right|^{\phi}\left|e^{m t}\right|\left|e^{-n(t-\tau)}\right| e^{p|t-\tau|}
$$

and, by (18) and (15),

$$
\left|{ }^{*} g^{h, 1, j}(t)\right|=|d(t)|\left|\tilde{\tilde{g}}^{h, 1, j}(t)\right|<d R^{\prime \prime}|t| \phi\left|e^{m t}\right|\left|e^{-n t}\right| e^{p|t|} \text {. }
$$

Thus, provided $R_{0}$ is taken $\geqq d R^{\prime \prime}$, inequalities (22) and (22a) are seen to be

$\dagger \rho^{\prime}$ is the least absolute value of the non-zero roots of the characteristic equation $E_{1}(\rho)=0$. 
true for $w=1$. Suppose that, for some $R_{1}$, for $t$ in $P$ and for $R \tau \geqq 0$,

$$
\begin{aligned}
& \left.\quad{ }_{\beta}^{* h, \sigma}(t, \tau)\left|<R_{1}\right| t\right|^{\phi}\left|e^{\sigma m t}\right|\left|e^{-n(t-r)}\right| e^{\rho|t-\tau|}, \\
& \left|{ }^{*} g^{h, \sigma, j}(t)\right|<R_{1}|t|^{\phi}\left|e^{\sigma m t}\right|\left|e^{-n t}\right| e^{\rho|t|} \\
& (\sigma=1, \cdots, w-1 ; 2 \leqq w \leqq p) .
\end{aligned}
$$

By (24), (24a), (18), (18a), (16), (15) from (19) and (19a) we then would have

$$
\begin{gathered}
\left|{ }_{\beta}^{*} C_{\zeta}^{h, w}(t, \tau)\right|<|t|^{\phi}\left|e^{w m t}\right|\left|e^{-n(t-\tau)}\right| e^{\rho|t-\tau|} R_{w}(t), \\
\left|{ }^{*} g^{h, w, j}(t)\right|<|t|^{\phi}\left|e^{w m t}\right|\left|e^{-n t}\right| e^{\rho|t|} R_{w}(t),
\end{gathered}
$$

where, for $R t \geqq 0$,

$$
R_{w}(t)=d\left(p d R_{1}+R^{\prime \prime}\left|e^{-(w-1) m t}\right|\right) \leqq d\left(p d R_{1}+R^{\prime \prime}\right) .
$$

On noting that $w$ may assume only a finite number of values it is concluded that there exists a sufficiently great $R_{0}$ so that the inequalities (22) and (22a) all hold for $w=1,2, \cdots, p$.

Thus, the following Lemma has been proved.

LEMMA 8. The formal series ${ }_{h} \tilde{\eta}_{w}{ }^{j-1}(t)(3 a)$, associated with the series $(1 ; \S 9)$ by means of $(1 \mathrm{a} ; \S 9)$, satisfy a certain integral system $\left(\mathrm{B}_{3}\right)$. The coefficients of this system are defined by convergent series (20), (20a); they are functions meromorphic in $t$, whose only finite t-singularities are poles at the non-zero roots of the characteristic equation $E_{1}(t)=0(c f .(5 ; \S 8))$. The ${ }_{\beta}^{*} c_{5}^{h, w}(t, \tau)$ are entire in $\tau$. Essential properties of these coefficients at $(t=0, \tau=0)$ are given by (21). On the other hand, for $t$ in $P$ and for $t$ in $S$ (cf. definitions at the beginning of this section), the coefficients of $\left(\mathrm{B}_{3}\right)$ satisfy inequalities (22) and (22a).

12. The Main Theorem for difference equations. The series ${ }_{h} \tilde{\eta}_{w}{ }^{j-1}(t)$ $(3 a ; \S 11)$, referred to in Lemma $8(\$ 11)$, are formal solutions of a system of integral equations for which all the conditions of Lemma 4 (\$7) are satisfied. Accordingly, by virtue of the latter lemma, the ${ }_{h} \tilde{\eta}_{w}{ }^{j-1}(t)$ converge in some vicinity of $t=0$, thus representing solutions, analytic at $t=0$, of the system $\left(\mathrm{B}_{3}\right.$; $\S 11)$. Such a system $\left(\mathbf{B}_{3}\right)$ exists for every $j(j=1, \cdots, \phi)$.

The following lemma will be now demonstrated.

LEMMA 9. Suppose that a system of integral equations $\left(\mathbf{B}_{8} ; \S 11\right)$ is given which satisfies the conditions of Lemma 8. The elements ${ }_{h} \tilde{\eta}_{w^{-1}}{ }^{-1}(t)(h=0, \cdots$, $j-1 ; w=1, \cdots, p)$, associated with any particular set of solutions of $\left(\mathrm{B}_{3}\right)$ (they are analytic at $t=0)$, have analytic continuations in the regions $P$ and $S$ 
(cf. definitions at the beginning of $\$ 11),{ }^{*}$ for which the following inequalities hold.

Along every line $R t=t_{1}$ in $S$, for $C$ and $q$ sufficiently great and independent of the position of the line, we have

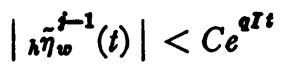

$$
\begin{aligned}
& (h=0, \cdots, j-1 ; w=1, \cdots, p) .
\end{aligned}
$$

When $m$ of $(4 \mathrm{a} ; \S 8)$ is zero, then, along every ray $\angle t=\bar{t}$ in $P$,

$$
\left|{ }_{h} \tilde{\eta}_{w}^{j-1}(t)\right|<C e^{q|t|} \quad(h=0, \cdots, j-1 ; w=1, \cdots, p) .
$$

Consider a particular set of solutions of $\left(\mathrm{B}_{\mathbf{8}}\right)$ and choose $C$ so that

$$
\left|h \tilde{\eta}_{w: 0}^{j-1}\right|<C
$$$$
(h=0, \cdots, j-1 ; w=1, \cdots, p),
$$

and so that, for every $q \geqq 0$, we have

$$
\left|{ }_{h \tilde{\eta}_{w}^{j-1}}(t)\right|<C e^{q|I t|} \quad(h=0, \cdots, j-1 ; w=1, \cdots, p),
$$

when $t$ is in $S$ and $I t \leqq \rho^{0}$ ( $\rho^{0}$ some positive number) and also when $|t| \leqq a$. Suppose now that the part of the Lemma referring to the region $S$ is not true. There exists then a number $\rho^{\prime}>\rho^{0}$ such that the following holds. For $I t<\rho^{\prime}$, along every line $R t=t_{1}$, in $S$,

$$
\left|{ }_{h} \tilde{\eta}_{w}^{j-1}(t)\right|<C e^{q I t} \quad(h=0, \cdots, j-1 ; w=1, \cdots, p) ;
$$

on the other hand, for some $h=h^{\prime}, w=w^{\prime}$ and for some line $R t=t_{1}^{\prime}$ in $S$,

$$
\left|\Lambda^{\prime} \tilde{\eta}_{w^{\prime}}^{i-1}(t)\right|=C e^{Q \rho^{\prime}}
$$

$$
\left(t=t^{\prime}=t_{1}{ }^{\prime}+i \rho^{\prime}\right) \text {. }
$$

Consider now an integral of the second member of $\left(B_{3}\right)$. Write

$$
\int_{0}^{t^{\prime}}=\int_{0}^{t_{1}^{\prime}}+\int_{t_{1^{\prime}}}^{t_{1}^{\prime}+i p^{\prime}} \text {. }
$$

It is observed that, for all involved $\beta, \zeta, h, w$,

$$
\left|{ }_{\beta}^{*} C_{\xi}^{h, w}(t, \tau)\right|<s_{1} \quad(|t|,|\tau| \leqq a) ;
$$

moreover, for $R t \leqq a$,

$$
\left|e^{w m t}\right| \leqq e^{p m a}
$$

By (6) and (3)

$$
\left|\int_{0}^{a_{1}^{\prime}}\right|<a s_{1} C
$$

\footnotetext{
* Continuations exist, of course, in regions more extensive than $P$ and $S$.
} 
and, by $(22 ; \S 11),(6 a)$ and $(4)$

(7a)

$$
\left|\int_{t_{1}^{\prime}}^{t_{1}^{\prime}+i \rho^{\prime}}\right|<e^{q \rho^{\prime}} R_{0} C e^{\rho m a}\left|t^{\prime}\right| \cdot \int_{0}^{\rho^{\prime}} e^{-(q-\rho)\left(\rho^{\prime}-\sigma\right)} d \sigma .
$$

From $(7 a)$, in view of $(6 b ; \S 7)$ we further have

$$
\left|\int_{t_{1}^{\prime}}^{t_{1^{\prime}}+i \rho^{\prime}}\right|<e^{q \rho^{\prime}} R_{0} C e^{p m a}\left|t^{\prime}\right|+\frac{1}{q-\rho},
$$

provided $q>\rho$. Furthermore, it is noted that

$$
\left|t^{\prime}\right|<\bar{a} \rho^{\prime} \quad\left(\bar{a}=\frac{1}{\rho^{0}}\left[a^{2}+\left(\rho^{0}\right)^{2}\right]^{1 / 2}\right)
$$

where $\bar{a}$ is independent of $t^{\prime}=t_{1}^{\prime}+i \rho^{\prime}$, provided $t^{\prime}$ is in $S$ and $\rho^{\prime}>\rho^{0}$. By (6a) and (9) from (22a) it follows that, for all involved $h, w, j$,

$$
\left|*^{k, w, j}\left(t^{\prime}\right)\right|<R_{0} e^{p m a} e^{\overline{p a \rho^{\prime}}}\left|t^{\prime}\right| \phi .
$$

Thus, in view of (7), (8), (5) and (10) on making use of (4a) and of $\left(B_{3}\right)$, we would have

$$
\left|t^{\prime}\right| \oplus C e^{q \rho^{\prime}}<C \phi p a s_{1}+C R_{0} \phi p e^{p m a} e^{q \rho^{\prime}}\left|t^{\prime}\right| \downarrow \frac{1}{q-\rho}+R_{0} e^{p m a} e^{p \overline{a \rho^{\prime}}}\left|t^{\prime}\right| \downarrow .
$$

Since $\rho^{\prime}>\rho^{0}$, this inequality would imply that

$$
1<\frac{\phi p a s_{1} e^{-q \rho^{0}}}{\left(\rho^{0}\right)^{\phi}}+\frac{R_{0} \phi p e^{p m a}}{q-\rho}+\frac{R_{0}}{C} e^{p m a} e^{-(q-p \bar{a}) \rho^{\prime}}=f(q) .
$$

Now, $f(q)$ approaches zero as $q \rightarrow \infty$. Hence for $q$ sufficiently great, so that $1 \geqq f(q)$, there arises a contradiction. Accordingly, the part of the Lemma relating to the strip $S$ has been demonstrated.

It remains to examine the case when $m=0$. We again consider a particular set of solutions of $\left(B_{3}\right)$ and we take $C$ so that (2) holds. Then, for some positive $r^{0}$ and for every $q \geqq 0$,

$$
\left|{ }_{h} \tilde{\eta}_{w}^{j-1}(t)\right|<C e^{q|t|} \quad(h=0, \cdots, j-1 ; w=1, \cdots, p)
$$

when $|t| \leqq r^{0}$. Assume that the part of the Lemma concerning the region $P$ does not hold. Then there will exist a number $r^{\prime}, r^{\prime}>r^{0}$, such that the following will be true. For $|t|<r^{\prime}$, along every ray $\bar{t}(=\angle t)$ in $P$,

$$
\left|{ }_{h} \tilde{\eta}_{w}^{j-1}(t)\right|<C e^{q|t|} \quad(h=0, \cdots, j-1 ; w=1, \cdots, p) .
$$

On the other hand, for some $h=h^{\prime}, w=w^{\prime}$, and for some ray $\angle t=\bar{t}^{\prime}$, in $P$, 


$$
\left|{ }_{h}^{\prime} \tilde{\eta}_{w^{\prime}}^{j-1}(t)\right|=C e^{q|t|}
$$

$\left(t=t^{\prime}=r^{\prime} e^{i i^{\prime}}\right)$.

From $\left(\mathrm{B}_{3}\right)$, by virtue of $(13),(13 \mathrm{a}),(22 ; \S 11)$ and $(22 \mathrm{a} ; \S 11)$, it would follow that

$$
\begin{aligned}
\left|t_{h^{\prime}}^{\phi} \eta_{w^{\prime}}^{j-1}(t)\right|= & |t|^{\phi} C e^{q|t|}<p \phi \int_{0}^{r^{\prime}} R_{0}|t|^{\phi} e^{p(|t|-|\tau|)} C e^{q|\tau|} d|\tau| \\
& +R_{0}|t| \phi e^{\rho|t|}
\end{aligned}
$$

here $t=t^{\prime}$ and integration is along the ray $\angle t=t^{\prime}$. In view of $(6 \mathrm{~b} ; \S 7)$ and since $r^{\prime}>r^{0},(14)$ implies that

$$
1<\frac{R_{0} p \phi}{q-\rho}+\frac{R_{0}}{C} e^{-(q-\rho) r^{0}}=g(q) .
$$

On noting that $g(q) \rightarrow 0$, as $q \rightarrow \infty$, again a contradiction is seen to arise, when $q$ is taken sufficiently great so that $1 \geqq g(q)$. Thus, the Lemma has been demonstrated completely.

Consider some line $R t=t_{1}$ in $S$. The formal series $(1 \mathrm{a} ; \$ 10)$ give rise to analytic functions $(3 ; \S 11)$

$$
{ }_{h} \eta_{w}^{j-1}(x)=\int_{0}^{\infty}{ }_{h} \tilde{\eta}_{w}^{j-1}(t) e^{t x} d t \quad(h=0, \cdots, j-1 ; w=1, \cdots, p),
$$

where the integration is extended in $S$ (cf. beginning of $\$ 11$ ) and the ${ }_{h} \tilde{\eta}_{w}{ }^{j-1}(t)$ are functions of a set referred to in Lemma 9. In fact, let $H$ denote an $x$-half plane for which

$$
R(i x)=|x| \cos \left(\frac{\pi}{2}+\bar{x}\right)<-q^{\prime}<0 \quad\left(\bar{x}=\angle x ; q^{\prime}>q\right) .
$$

For $x$ in $H$, in consequence of (1), the integrals (16) are seen to be absolutely convergent, when the path of integration is extended in $S$.

Similarly, when $m$ of $(4 \mathrm{a} ; \S 8)$ is zero and integration in (16) is along a ray $\angle t=\bar{t}$ in $P$, the integrals $(16)$ are observed to be absolutely convergent in a half plane $H[\bar{t}](\mathrm{cf} .(9 ; \S 7))$; this fact is a consequence of $(1 \mathrm{a})$.

It is also noted that when $x$ is in $H$ condition $(1 ; \S 11)$ is necessarily satisfied. On the other hand, for $x$ in $H[\bar{t}]$ ( ray $\bar{t}$ in $P$ ), this condition will also be satisfied, by virtue of $(9 ; \S 7)$.

When $m=0$ the condition $(5 ; \S 11)$ is satisfied along any ray $\angle t=\bar{t}$, in $P$, provided $x$ is in $H[\bar{t}]$ and $q^{\prime}$ of $(9 ; \S 7)$ is sufficiently great. It remains to consider the case when $m$ is not necessarily zero. The function $f(t, x)$, within the brackets of $(5 ; \S 11)$, vanishes for $t=0$. We have to show that 


$$
\lim _{\mapsto \infty} f(t, x)=0
$$

here $t \rightarrow \infty$ along any line $R t=t_{1}$, in $S$. On writing

$$
\begin{aligned}
f(t, x) & =f_{1}(t, x)+f_{2}(t, x), \\
f_{1}(t, x) & =e^{t x} \int_{0}^{t_{1}} e^{k t}{ }_{k}^{j-1}(t) d t^{(H)}, \\
f_{2}(t, x) & =e^{t x} \int_{t_{1}}^{t} e^{k t}{ }_{k} \tilde{\eta}_{w}^{j-1}(t) d t^{(H)},
\end{aligned}
$$

we note that, in view of $(17), \lim f_{1}(t, x)=0$. As to the function $f_{2}(t, x)$, it is found without difficulty that, by virtue of (1) and (17), ${ }^{*}$ its limit along the line $R t=t_{1}$ is also zero. Thus, $(5 ; \S 11)$ holds in $S$.

Accordingly, all those developments which originally were of a formal character are now seen to be justified.

Application of fundamental theorems of Nörlund, referred to in $\$ 7$, is possible. In fact, with the integration extended in $S$, an integral (16) may be expressed as

$$
{ }_{h} \eta_{w}^{j-1}(x)={ }_{h} e_{w}^{j-1}(x)+{ }_{h} f_{w}^{j-1}(x) e^{x t_{1}}
$$

where

$$
{ }_{h} e_{w}^{j-1}(x)=\int_{0}^{t_{1}} e^{t x}{ }_{h}{ }_{n}^{j-1}(t) d t
$$

$\left[0<\epsilon \leqq t_{1} \leqq a ; \epsilon\right.$ a constant used in the definition of $\left.S\right]$ and

$$
{ }_{h} f_{w}^{j-1}(x)=\int_{t_{1}}^{t_{1}+i \infty} e^{\left(t-t_{1}\right) x}{ }_{h} \tilde{\eta}_{w}^{j-1}(t) d t .
$$

The function (18a) is entire. On the other hand, in view of Nörlund's results and in view of the properties established, in $S$, for the ${ }_{h} \tilde{\eta}_{w}{ }^{j-1}(t)$, the ${ }_{h} f_{w}^{j-1}(x)$ are seen to be expressible by series

$$
\begin{aligned}
{ }_{n} f_{w}^{j-1}(x)=\sum_{s=0}^{\infty} \frac{{ }_{h} f_{w}^{j-1}\left(t_{1}\right)}{x(x+i \gamma)(x+2 i \gamma) \cdots(x+s i \gamma)} \\
\quad(\gamma>0, \text { sufficiently great }),
\end{aligned}
$$

convergent in a plane $H$ (cf. (17)); that is, convergent for

$$
I x>B>0
$$

( $H$ sufficiently great).

\footnotetext{
* And also because along the line $R t=t_{1}$, in $S, \angle t \rightarrow \pi / 2$.
} 
When $m$ of $(4 \mathrm{a} ; \S 8)$ is zero (that is, when the solutions under considerations are associated with the $\mu$-group (cf. \$8) whose $\mu$ 's are greatest) consideration of integrals (16) (with integrations along a ray in $P$ ) leads to convergent factorial series developments analogous to those obtained in $\$ 7$.

Thus, we have found a set of analytic solutions ${ }_{h} \eta_{w}{ }^{j-1}(x)(h=0, \cdots, j-1$; $w=1, \cdots, p)$ of the "mixed" difference system $\left(\mathrm{B}_{2}\right)(\$ 10)$. Such results are established for $j=1, \cdots, \phi$. Now the $\phi$ formal solutions, under consideration, of $\left(B_{1}\right)(\$ 8)$ are given by $(1 ; \S 10)$. In view of the established "summability" of the ${ }_{h} \eta_{w}{ }^{j-1}(x)$, the relations $(1 ; \S 10)$ yield analytic expressions of a set of $\phi$ linearly independent solutions of $\left(B_{1}\right)$. Consequently, we may formulate as follows the Main Theorem for difference equations.

THEOREM II. Let a difference equation (B) (\$1) be given. Suppose that corresponding to a root $\rho_{1}$, of multiplicity $\phi$, of one of the associated characteristic equations (there is one such equation for each $\mu$-group; cf. §8) there exists a linearly independent set of $\phi$ formal series solutions, all of normal type (\$8) and all forming one logarithmic group. Bring (B) to the corresponding form $\left(\mathrm{B}_{1}\right)(\$ 8)$ and let $E_{1}(\rho)=0(5 ; \S 8)$ be the characteristic equation, just referred to, of $\left(\mathrm{B}_{1}\right)$. For every $t_{1}$, such that $\epsilon \leqq t_{1} \leqq a(0<\epsilon<a ; a$ sufficiently small $)$, the following is true.

$\left(\mathrm{B}_{1}\right)$ possesses a set of $\phi$ linearly independent analytic solutions

$$
\begin{array}{r}
y_{j}(x)=e^{Q(x)} x \sum_{h=0}^{r-1} \log ^{h} x\left[{ }_{h} \eta_{0}^{j-1}+\sum_{w=1}^{p} x^{(p-w) / p}\left({ }_{h} e_{w}^{j-1}(x)+{ }_{h} f_{w}^{j-1}(x) e^{x t_{t}}\right\}\right] \\
\left(j=1, \cdots, \phi ; Q(x) \text { a polynomial in } x^{1 / p}\right),
\end{array}
$$

where the ${ }_{n} e_{w}{ }^{i-1}$ are entire functions of the type (18a), while the ${ }_{n} f_{w}{ }^{i-1}(x)$ are factorial series of the form (19), convergent for $I x>H>0$ ( $H$ sufficiently great). The functions

$$
{ }_{n} e_{w 0}^{j-1}(x)+{ }_{n} f_{w}^{j-1}(x) e^{x t_{1}}
$$

are expressible by convergent Laplace integrals of the form (16). There exist corresponding developments for a half plane $I x<-H_{1}<0$.

When the solutions, under consideration, correspond to the $\mu$-group whose $\mu$ 's are the greatest, the following will hold for every $\bar{t}(-\pi / 2<\bar{t}<\pi / 2)$, not coincident with a value of an angle of a non-zero root of $E_{1}(\rho)=0 . *$

$\left(\mathrm{B}_{1}\right)$ possesses a set of $\phi$ linearly independent analytic solutions of the form $(11 ; \S 7)$, where $Q(x)$ contains no powers of $x$ higher than the first. Similar developments exist for $\pi / 2<\bar{t}<3 \pi / 2$.

\footnotetext{
* It is supposed that $\rho_{1}=0$; this entails no loss of generality.
} 
In so far as the corresponding solutions of (B) are concerned, we need only to adjoin a suitable factor $\exp (\mu x \log x)$ to the expressions given in the above theorem.

The theorem is not capable of extension in the sense that even normal formal solutions of (B) do not in all cases lead to convergent factorial series developments, if corresponding to the multiple root, in question, there is more than one logarithmic group. This point will be demonstrated by means of the following example.

Let $L(y)=0$ be an equation $\left(B_{1}\right)(\S 8)$ of third order, with $p=1$ and with all the $\mu_{i}(\$ 8)$ zero. We shall take

$$
\begin{gathered}
d_{0,0}=1, \quad d_{1,0}=-3, \quad d_{2,0}=3, \quad d_{3,0}=-1, \\
d_{0,1}=d_{2,1}=0, \quad d_{1,1}=1, \quad d_{3,1}=-1
\end{gathered}
$$

and

$$
d_{3-k, s}=0 \quad(k=0,1,2,3 ; s=2,3, \cdots),
$$

except that $d_{3,8}=b<0$. This equation has a single characteristic equation with a triple root $\rho=0$. By direct substitution it can be verified that $L(y)$ is satisfied by a formal normal solution*

$$
\begin{aligned}
y(x) & ={ }_{\circ \eta^{0}}(x)=1+{ }_{\circ \eta_{1}^{0}}^{0}(x), \\
o \eta_{1}^{0}(x) & =\sum_{\lambda=0}^{\infty}{ }_{0 \eta_{\lambda+1}^{0} x^{-(\lambda+1)} .}
\end{aligned}
$$

It can be shown that the number of logarithmic groups is greater than one.

Corresponding to $\left(B_{2}\right)(\$ 10)$ we have

$$
T_{0,1}^{0} \equiv \sum_{k=0}^{8}{ }_{0}^{0,1} a_{1, k}^{0,1}(x) \quad 0 \eta_{1}^{0}(x+k)=g^{0,1,1}(x),
$$

where

$$
\begin{aligned}
& { }_{0} a_{1, k}^{0,1}(x)=\sum_{s=0}^{\infty}{ }_{0} a_{1, k: s}^{0,1} x^{-8}, \quad{ }_{0}^{0,1, k: \ell}=d_{3-k, e}, \\
& g^{0,1,1}(x)=-\sum_{s=1}^{\infty} g_{s}^{0,1,1} x^{-s} ;
\end{aligned}
$$

here

$$
g_{8}^{0,1,1}=0 \quad(s \neq 3), \quad g_{3}^{0,1,1}=b .
$$

* Use is made of a notation conforming with that employed in $\$ \S 8,9,10,11,12$. 
Let $\angle t=\bar{t}$ be any ray, extending from $t=0$ and not coincident with either half of the axis of $t$-imaginaries. Let integrations be along such a ray. Corresponding to $(3 ; \S 11)$ we write, formally,

$$
{ }_{0}^{0} \eta_{1}(x)=\int_{0}^{\infty}{ }_{0} \bar{\eta}_{1}^{0}(t) e^{t x} d t \quad\left(R\left(e^{i \bar{t}} x\right)<-q^{\prime}<0\right),
$$

where $q^{\prime}$ is sufficiently great and

$$
0 \tilde{\eta}_{1}^{0}(t)=\sum_{\nu=0}^{\infty} \eta_{\nu} t^{\prime} \quad\left(\eta_{\nu}=\frac{(-1)^{\nu+1}}{\nu !} \underset{0 \eta_{v+1}^{0}}{0}\right) .
$$

If it were demonstrated that (25a) diverges, impossibility of representing $y(x)$ in terms of a convergent factorial series would have been established. This follows by a reasoning analogous to that employed for a similar purpose at the end of $\$ 7$.

Use of the difference equation appears to be impracticable in proving divergence of the series (25). However, a certain integral equation will serve this purpose. Substitution of $(25)$ in $(24)$ (compare with $(8 ; \$ 11)$ ) leads to the equation

$$
\left(e^{t}-1\right)^{3} \circ \tilde{\eta}_{1}^{0}(t)=\int_{0}^{t}\left(e^{2 \tau}-1+\frac{b}{2}(\tau-t)^{2}\right){ }_{0} \tilde{\eta}_{1}^{0}(\tau) d \tau+\frac{b}{2} t^{2}
$$

which yields the following relations for the coefficients of the series (25a):

$$
\frac{1}{r} \eta_{r-2}=\sum_{H=0}^{r-3} f_{H}(r) \eta_{H} \quad(r \geqq 3), \quad \eta_{0}=-\frac{b}{2},
$$

where

$$
\begin{aligned}
f_{r-3}(r) & =\frac{5}{6}-\frac{2}{r}-\frac{b}{r(r-1)(r-2)}, \\
f_{H}(r) & =\frac{1}{(r-H) !} \delta_{0}{ }^{r-H}-\frac{1}{(r-H-1) ! r !} 2^{r-B-1} \quad(0 \leqq B \leqq r-4) .
\end{aligned}
$$

Since $b<0, f_{r-3}(r)>0(r \geqq 3)$. We have $\delta_{0}^{r-B}=3^{r-B}-3\left(2^{r-B}\right)+3$. Thus, for $0 \leqq H \leqq r-4$,

$$
f_{H}(r)=\frac{3^{r-B-1}}{(r-H) !}\left(f_{H}^{\prime}(r)-f_{H^{\prime \prime}}^{\prime \prime}(r)\right)
$$

where 


$$
\begin{aligned}
& f_{H}^{\prime}(r)=3\left[1-2\left(\frac{2}{3}\right)^{-H-1}+\frac{1}{3^{r-B-1}}\right]>\frac{11}{9}, \\
& f_{H}^{\prime \prime}(r)=\frac{r-H}{r}\left(\frac{2}{3}\right)^{r-H-1} \leqq \frac{8}{27} .
\end{aligned}
$$

Accordingly, from (27b) it follows that, for $0 \leqq H \leqq r-4$,

$$
f_{H}(r)=\frac{3^{r-H-1}}{(r-H) !} \zeta_{H}(r), \quad \zeta_{H}(r)>\frac{25}{27} .
$$

With the coefficients $f_{H}(r)$ in the second members of (27) all positive, it is seen that the $\eta_{\nu}$ of (25a) are uniquely determined, positive numbers. Hence relations (27) would imply that

$$
\frac{\eta_{r-2}}{r}>f_{r-3}(r) \eta_{r-3} \quad(r \geqq 4) .
$$

Now, by (27a), $r f_{r-3}(r) \rightarrow \infty$ as $r \rightarrow \infty$. Consequently, inequalities (28) lead to the conclusion that the series (25a) diverges for all $t(\neq 0)$. Thus, the normal formal solution of the example under consideration cannot be represented in terms of a convergent factorial series.

UNIVERSTTY OF ILLINOIS, URBANA, ILL. 